\title{
Tetrahydrodibenzo[a,i]phenanthridin-5-yl)phenol as fluorescent probe for the detection of Aniline
}

\author{
Umamahesh Balijapalli, ${ }^{a}$ Saravanakumar Manickam, ${ }^{a}$ Krishnan Thirumoorthy, ${ }^{a}$ Karthikeyan Natesan \\ Sundaramurthy*b and Sathiyanarayanan Kulathu Iyer ${ }^{\mathrm{a}^{*}}$ \\ a *Department of Chemistry, School of Advanced Sciences, Vellore Institute of Technology University, \\ Vellore-632014, Tamil Nadu and India. E-mail: sathiya_kuna@hotmail.com \\ bDepartment of Chemistry, SRM Easwari Engineering College, Chennai 600089, Tamil Nadu, India.
}

\section{Table of contents}

Chart S1. Schematic representation and different paths for the detection of aniline S4 Figure S1. ORTEP structure of receptor P1 and P2

Figure S2. The energy diagram of ground state and excited state optimized geometries of P1, P2, PBC1 and PBC2 in aniline and TEA.

Figure S3. The energy diagram of planar and non-planar conformations of the ground state (GS) and the excited state (ES) P1 and P2 in aniline and TEA.

Figure S4. Frontier molecular orbitals distribution of PBC1 obtained with TEA (a) ground state and (b) excited state

S8

Figure S5. Changes observed in (a) aromatic and (b) aliphatic region of ${ }^{1} \mathrm{H}$ NMR spectra

S9

Figure S6. Emission spectra of $\mathrm{P} 2$ and $\mathrm{PBC} 2$ recorded in various solvents

Figure S7. Emission spectra of P2 recorded with increasing the DMSO content in THF solvent S11

Figure S8. The fluorescence decay profiles of P2, PC2 and PBC2

Figure S9. (a) Changes in the absorbance (b) Changes in the fluorescence intensities of PB2 after the addition of different organic functional groups

S13

Figure S10. (a) Changes in the emission spectra of $\mathrm{P} 2$ after adding the mixture of $\mathrm{BF}_{3} \cdot \mathrm{OEt}_{2}$ and different organic functional groups

S14

Figure S11. Selectivity studies recorded using emission spectra by adding (a) various boron compounds and (b) mixture of aniline (An)+boron compounds

Figure S12. Emission titrations of PB2 performed with the addition of trimethylamine (TEA) S16

Figure S13. ORTEP structure of P2-BF4 adducts

Figure S14. Changes observed in fluorescence intensities of $\mathrm{P} 2$ as a function of Aniline- $\mathrm{BF}_{3} \cdot \mathrm{OEt}_{2} \mathbf{S 1 7}_{7}$

Figure S15. Changes observed in the fluorescence intensities of P2 as a function of [4-Aminobenzoic $\operatorname{acid}-\mathrm{BF}_{3} \cdot \mathrm{OEt}_{2}$ ] 
Figure S16. Changes observed in the fluorescence intensities of P2 as a function of [4-Methoxyaniline$\left.\mathrm{BF}_{3} . \mathrm{OEt}_{2}\right]$

Figure S17. Changes observed in the fluorescence intensities of P2 as a function of [2-aminopyridine$\left.\mathrm{BF}_{3} \cdot \mathrm{OEt}_{2}\right]$

S18

Figure S18. Changes observed in the fluorescence intensities of P2 as a function of [1-napthylamine$\left.\mathrm{BF}_{3} . \mathrm{OEt}_{2}\right]$

S19

Figure S19. Changes observed in the fluorescence intensities of P2 as a function of $\left[4,4^{\prime}-\right.$ diaminodiphenylmethane- $\left.\mathrm{BF}_{3} \cdot \mathrm{OEt}_{2}\right]$

Figure S20. Jobs plot

Figure S21. Emission titrations of $\mathrm{P} 2$ performed with the addition of $\mathrm{TEA}+\mathrm{BF}_{3} \cdot \mathrm{OEt}_{2}$ mixture $\quad$ S20

Figure S22. Time dependent emission titrations of $\mathrm{P} 2$ performed with 4 -aminobenzoic acid $+\mathrm{BF}_{3} \cdot \mathrm{OEt}_{2}$ mixture

Figure S23. Time dependent emission titrations of $\mathrm{P} 2$ performed with 4-methoxy aniline $+\mathrm{BF}_{3} \cdot \mathrm{OEt}_{2}$ mixture

Figure S24. Time dependent emission titrations of $\mathrm{P} 2$ performed with 1-napthylamine $+\mathrm{BF}_{3} . \mathrm{OEt}_{2}$ mixture

S22

Figure S25. Time dependent emission titrations of P2 performed with 2-aminopyridine $+\mathrm{BF}_{3} . \mathrm{OEt}_{2}$ mixture

Figure S26. Time dependent emission titrations of P2 with 4,4'-diaminodiphenylmethane $+\mathrm{BF}_{3} \cdot \mathrm{OEt}_{2}$ mixture

Figure S27. Time dependent emission titrations of $\mathrm{P} 2$ with diethylamine $+\mathrm{BF}_{3} \cdot \mathrm{OEt}_{2}$ mixture.

Figure S28. Time dependent emission titrations of $\mathrm{BF}_{3} \cdot \mathrm{OEt}_{2}$ with ethylamine $+\mathrm{BF}_{3} \cdot \mathrm{OEt}_{2}$ mixture $\mathbf{S 2 4}$

Figure S29. The plot of reaction time as a function of emission intensity given in bar diagram

Figure S30. The emission response of $\mathrm{P} 2$ as a function of various amines mixture

Figure S31. Changes in the emission intensities of $\mathrm{P} 2$ after adding $\mathrm{BF}_{3} \cdot \mathrm{OEt}_{2}$ and different aromatic primary amines

Figure S32. Effect of $\mathrm{pH}$ on the fluorescence intensity of P2

Figure SI-33 to 35 Fluorescence spectra of receptor P2 in the presence of various metal ions, organic acids and electron deficient organic substances

Figure S36. Changes in (a) absorbance and (b) emission intensities of P2 after adding $\mathrm{Zn}^{2+}$

Figure S37. Calibration plot of Aniline concentration versus Fluorescence intensity

Figure S38. Screening of various metal ions using absorption studies

${ }^{1} \mathrm{H},{ }^{13} \mathrm{C}$ NMR, COSY, HMQC spectrum of compounds P1 and P2 
${ }^{1} \mathrm{H}$ and ${ }^{13} \mathbf{C}$ NMR spectrum of $\mathrm{P} 2 \mathrm{ZnCl} 2$

SI Table S1 The comparisons of aniline sensor systems

SI Table S2 and S3 for crystal data and structure refinement for P1 and P2

S40

SI Table S4. The energy of ground state and excited state optimized geometries of P1, P2, PBC1 and PBC2 in aniline and TEA.

S45

SI Table S5. Cartesian coordinates (in $\AA$ units) of the optimized ground state geometries of P1 and P2 in gas phase

S45

SI Table S6. Cartesian coordinates (in $\AA$ units) of the optimized ground state geometries of P1 and P2 in aniline

S47

SI Table S7. Cartesian coordinates (in $\AA$ units) of the optimized ground state geometries of P1 and P2 in TEA

S48

SI Table S8. Cartesian coordinates (in $\AA$ units) of the optimized ground state geometries of PBC1 and $\mathrm{PBC} 2$ in aniline

SI Table S9. Cartesian coordinates (in $\AA$ units) of the optimized ground state geometries of PBC1 and PBC2 in TEA

SI Table S10. Number of imaginary frequencies obtained for optimized ground state geometries of P1, $\mathrm{P} 2, \mathrm{PBC} 1$, and $\mathrm{PBC} 2$

SI Table S11. The absolute energy of the optimized ground state $\left(\mathrm{S}_{0}\right)$ and singlet excited state $\left(\mathrm{S}_{1}\right)$ geometries of P1, P2, PBC1, and PBC2

S54

SI Table S12. Cartesian coordinates (in $\AA$ units) of the optimized singlet state geometries of P1 and P2 in aniline

S54

SI Table S13. Cartesian coordinates (in Å units) of the optimized singlet state geometries of P1 and P2 in TEA

SI Table S14. Cartesian coordinates (in $\AA$ units) of the optimized singlet state geometries of PBC1 and $\mathrm{PBC} 2$ in aniline

SI Table S15. Cartesian coordinates (in $\AA$ units) of the optimized singlet state geometries of PBC1 and PBC2 in TEA 
Chart S1. Schematic representation and different paths for the detection of aniline.
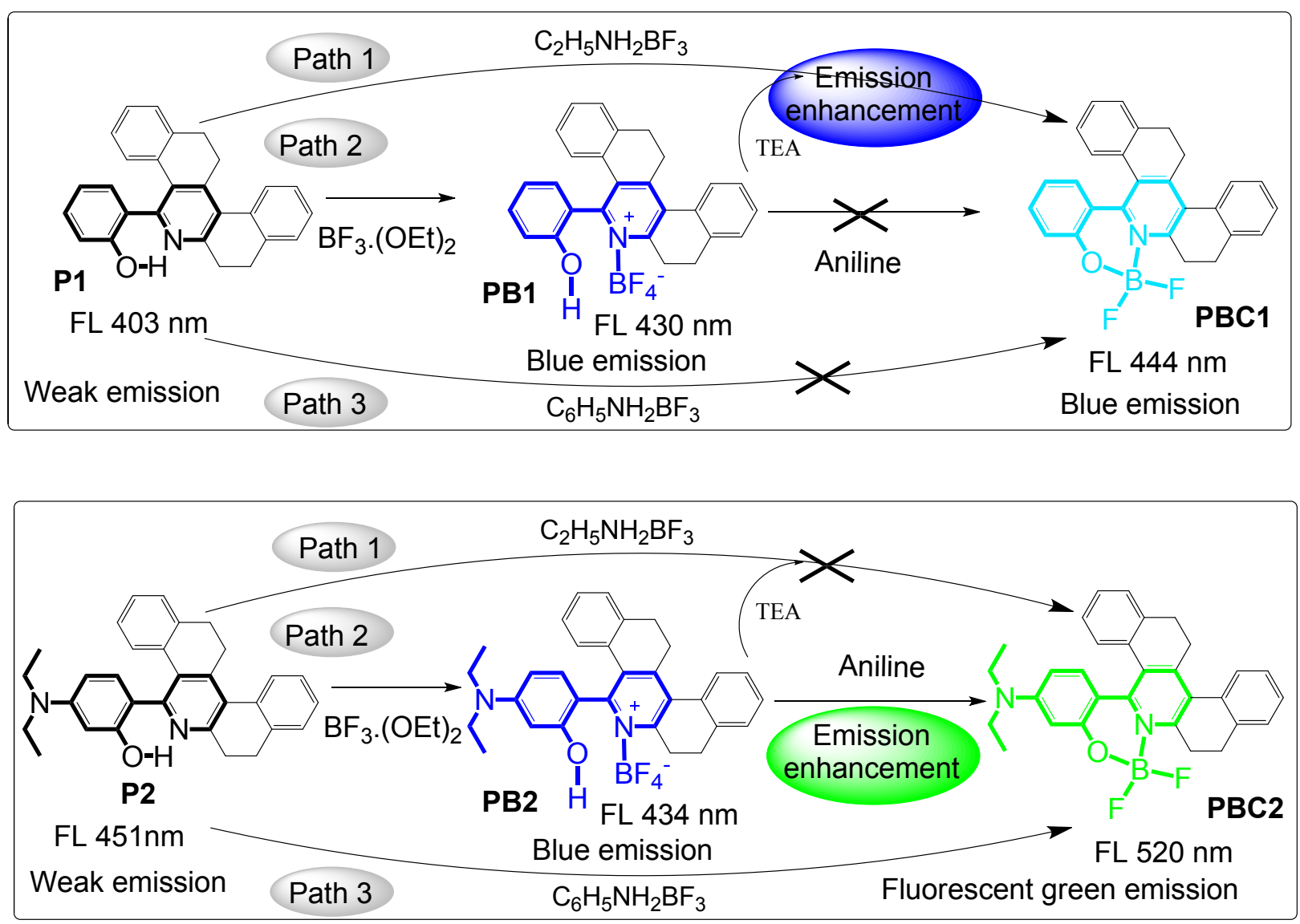
Figure S1. ORTEP structure of receptor P1 (a) $(C C D C=104929)$ and $\mathbf{P 2}(b)(C C D C=1049289$ ) with $30 \%$ ellipsoidal probability.

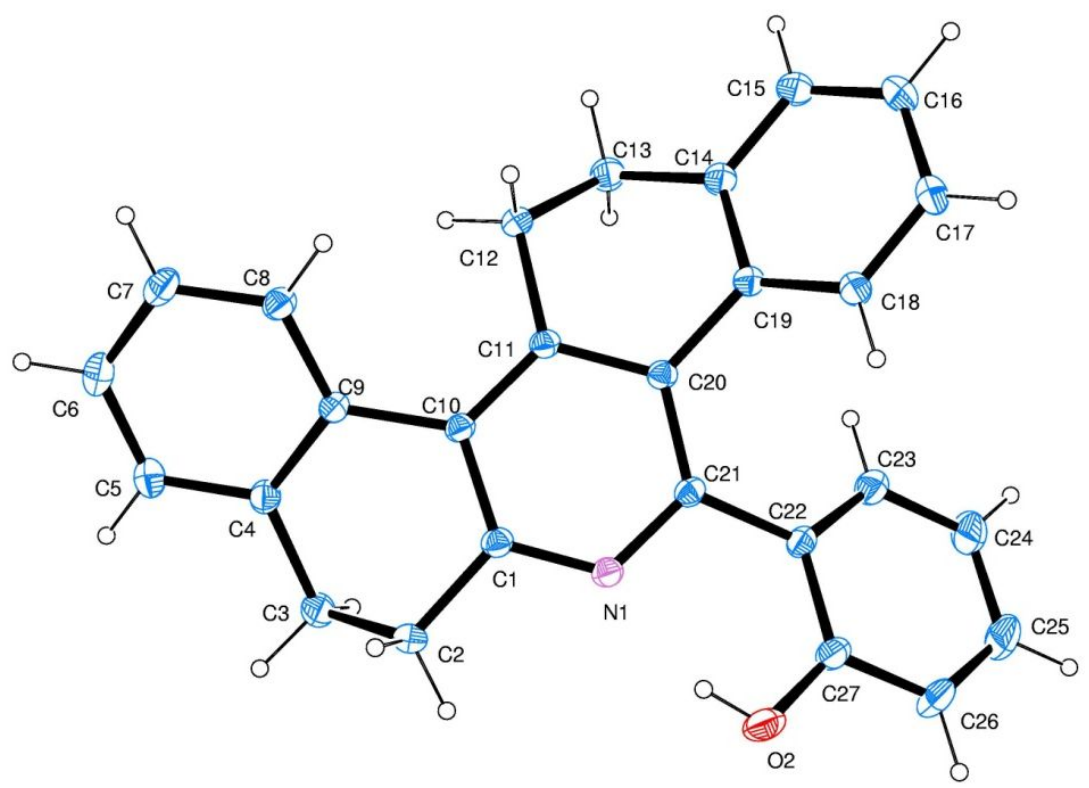

(a)

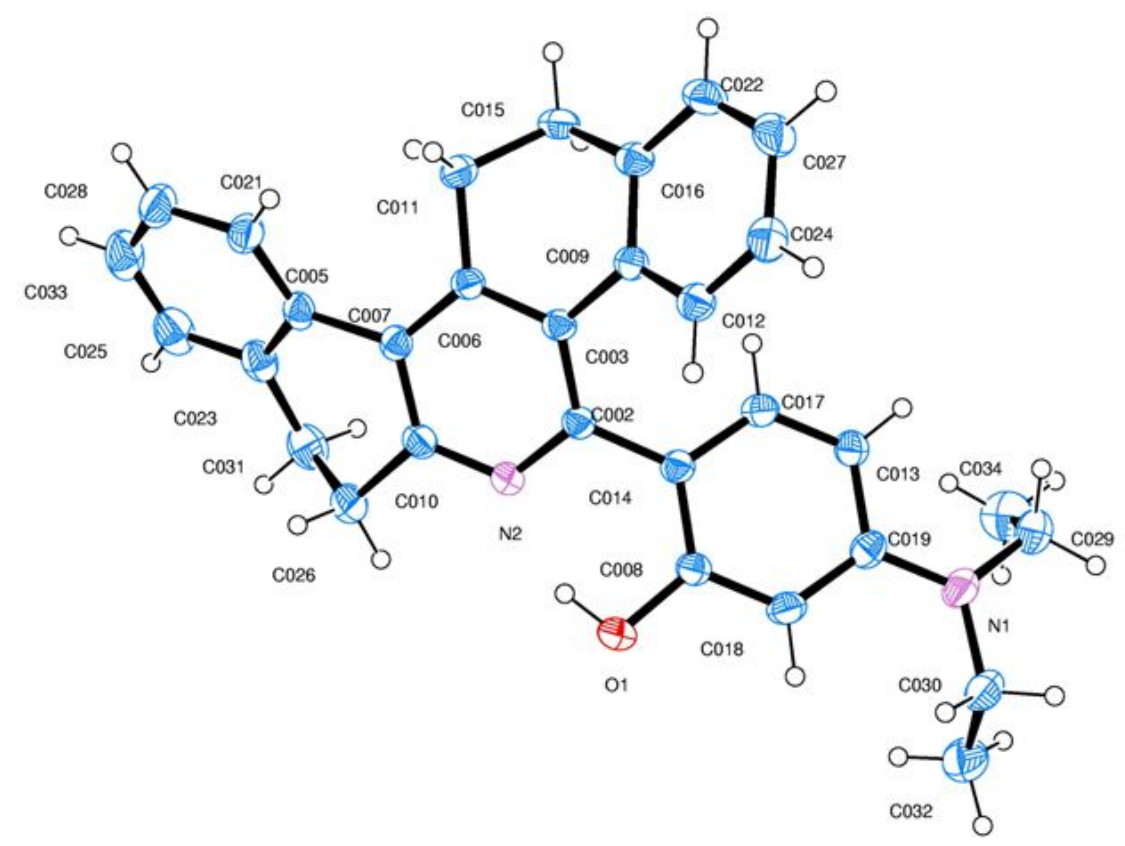

(b) 
Figure S2. The energy diagram of ground state and excited state optimized geometries of P1, P2, PBC1 and PBC2 in aniline and TEA. The optimization of ground state with DFT and the excited state with TDDFT were done with B3LYP/6-31+G* method. All the energies are expressed in $\mathrm{eV}$ as relative to the respective ground state. The electronic transitions shown in the diagram reflect the observed energy difference between the ground state and their corresponding excited state systems as given in table S1.

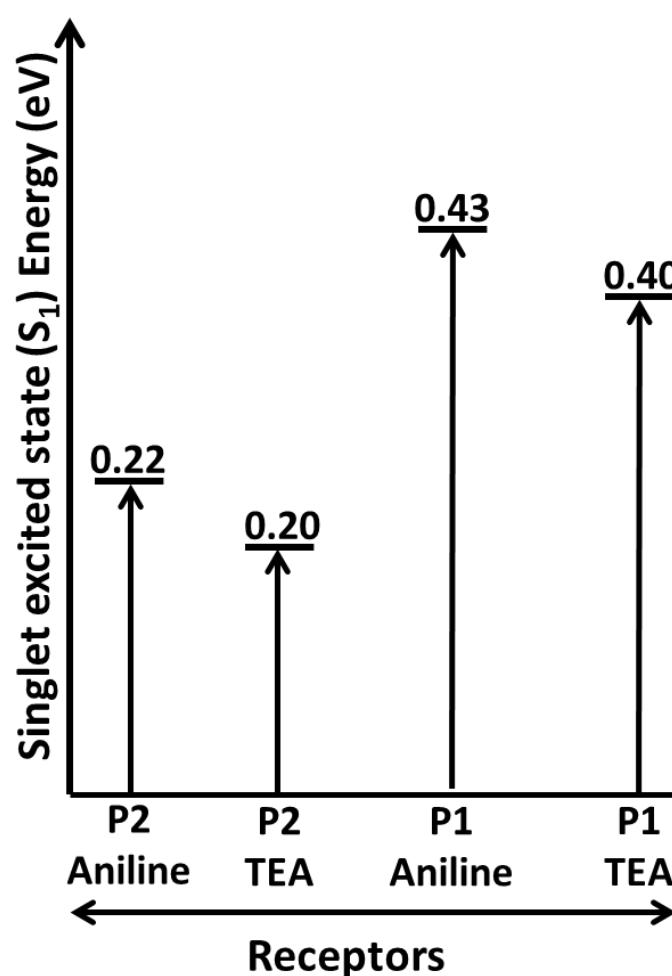

Receptors
0.56

个 
Figure S3. The energy diagram of planar and non-planar conformations of the ground state (GS) and the excited state (ES) $\mathbf{P 1}$ and $\mathbf{P 2}$ in aniline and TEA. The optimization of ground state with DFT and excited state with TDDFT were done with B3LYP/6-31+G* method. All the energies are given in atomic unit (Hartree).

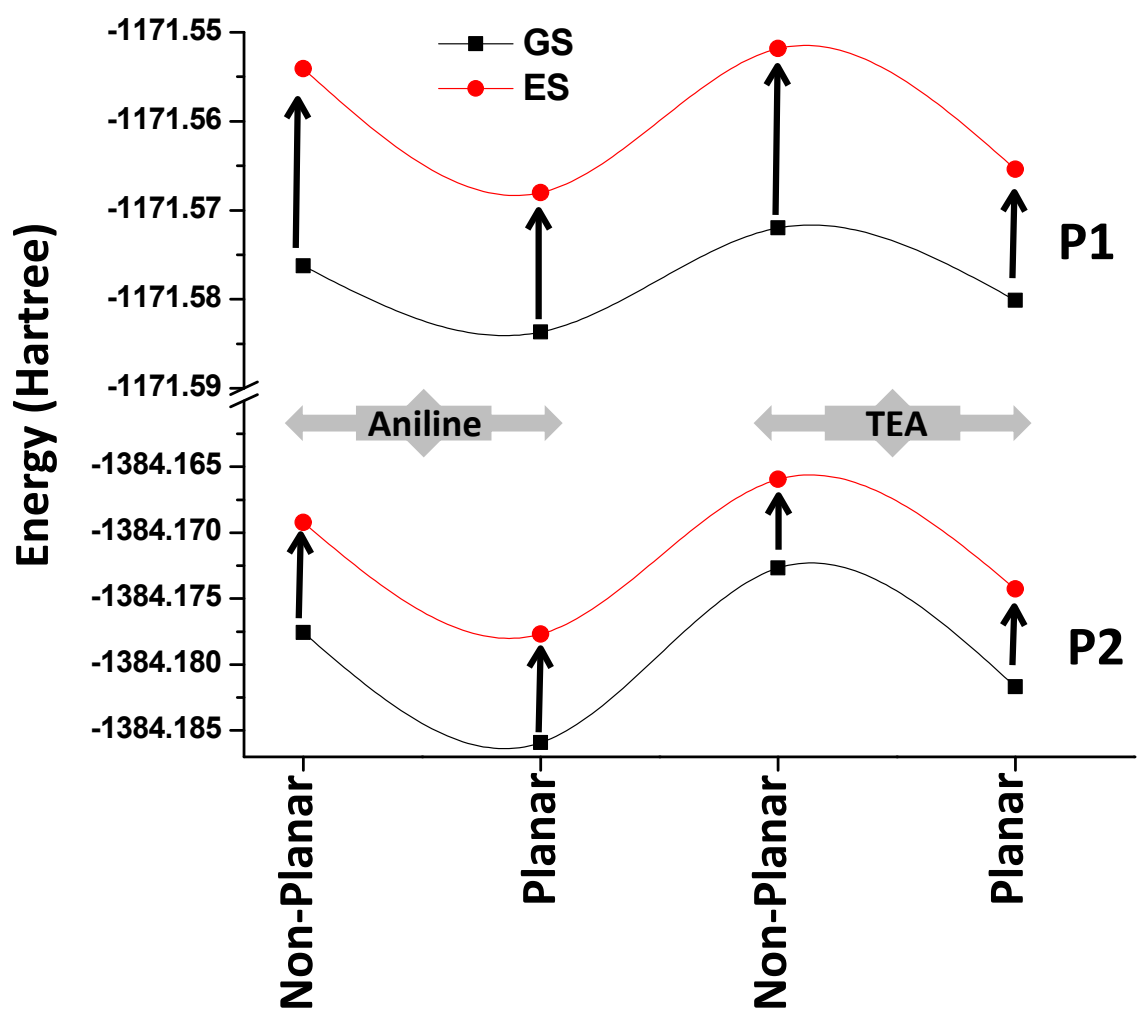


Figure S4. Frontier molecular orbitals distribution of PBC1 obtained with TEA (a) ground state and (b) excited state with B3LYP/6-31+G* level of theory.

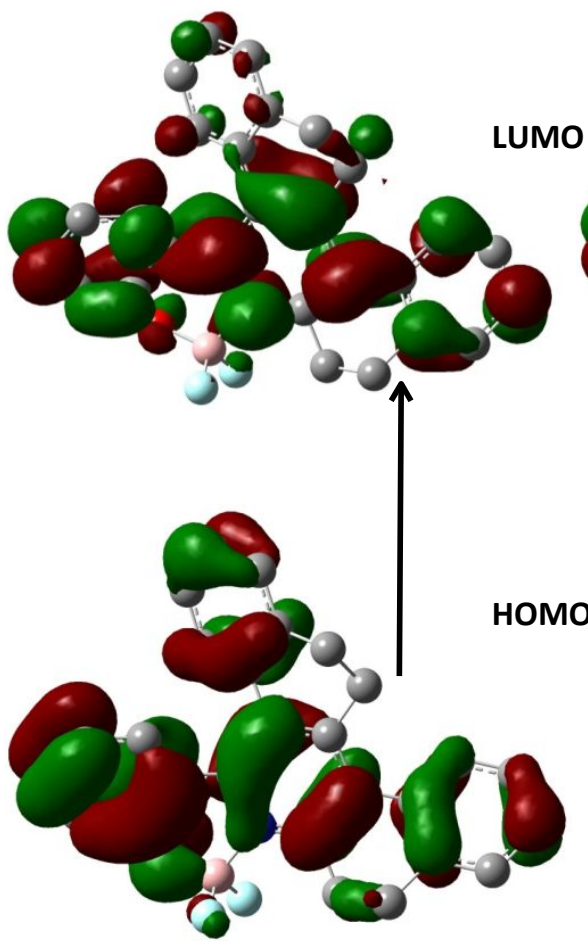

(a) Ground State PBC1 in TEA

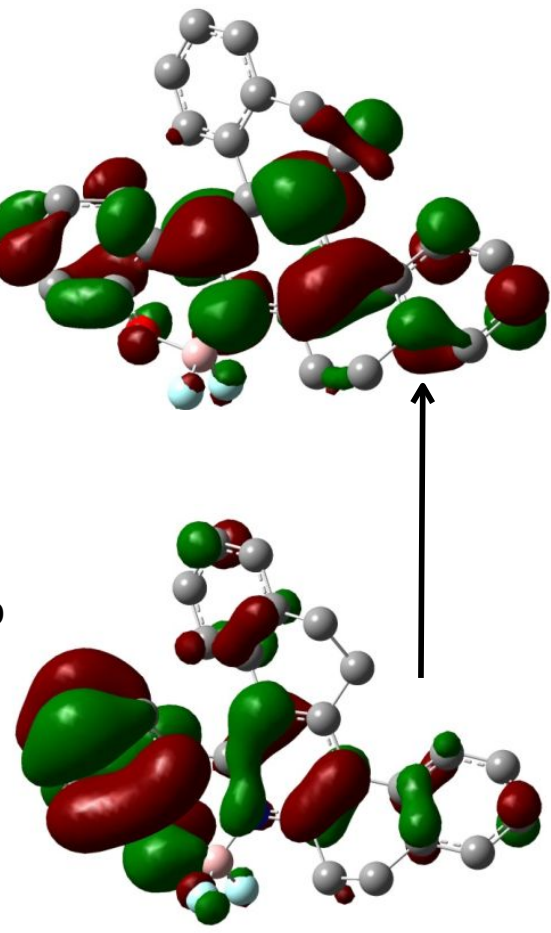

(b) Singlet Excited PBC1 in TEA 
Figure S5. Changes observed in (a) aromatic and (b) aliphatic region of ${ }^{1} \mathrm{H}$ NMR spectra.

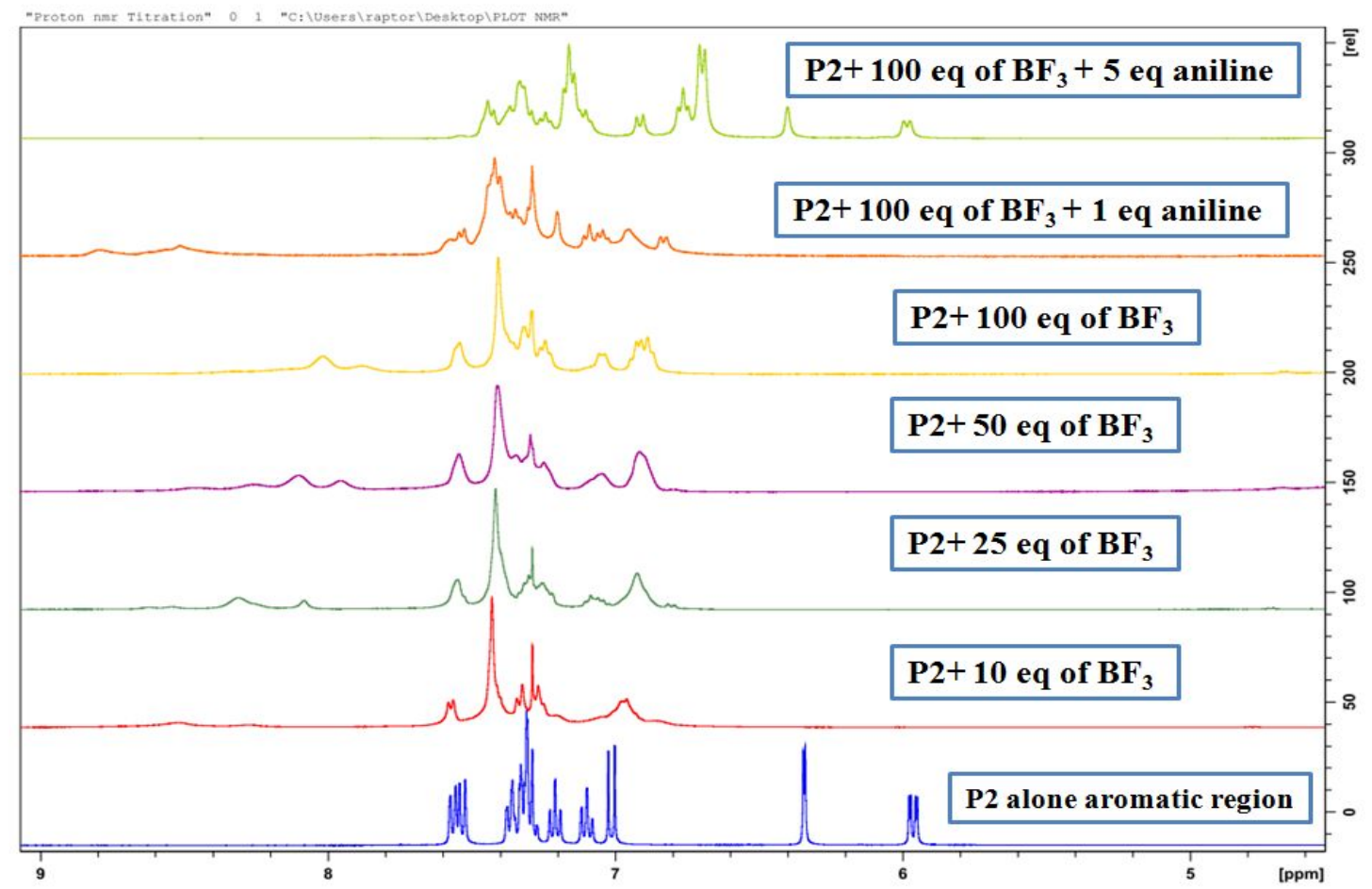

(a)

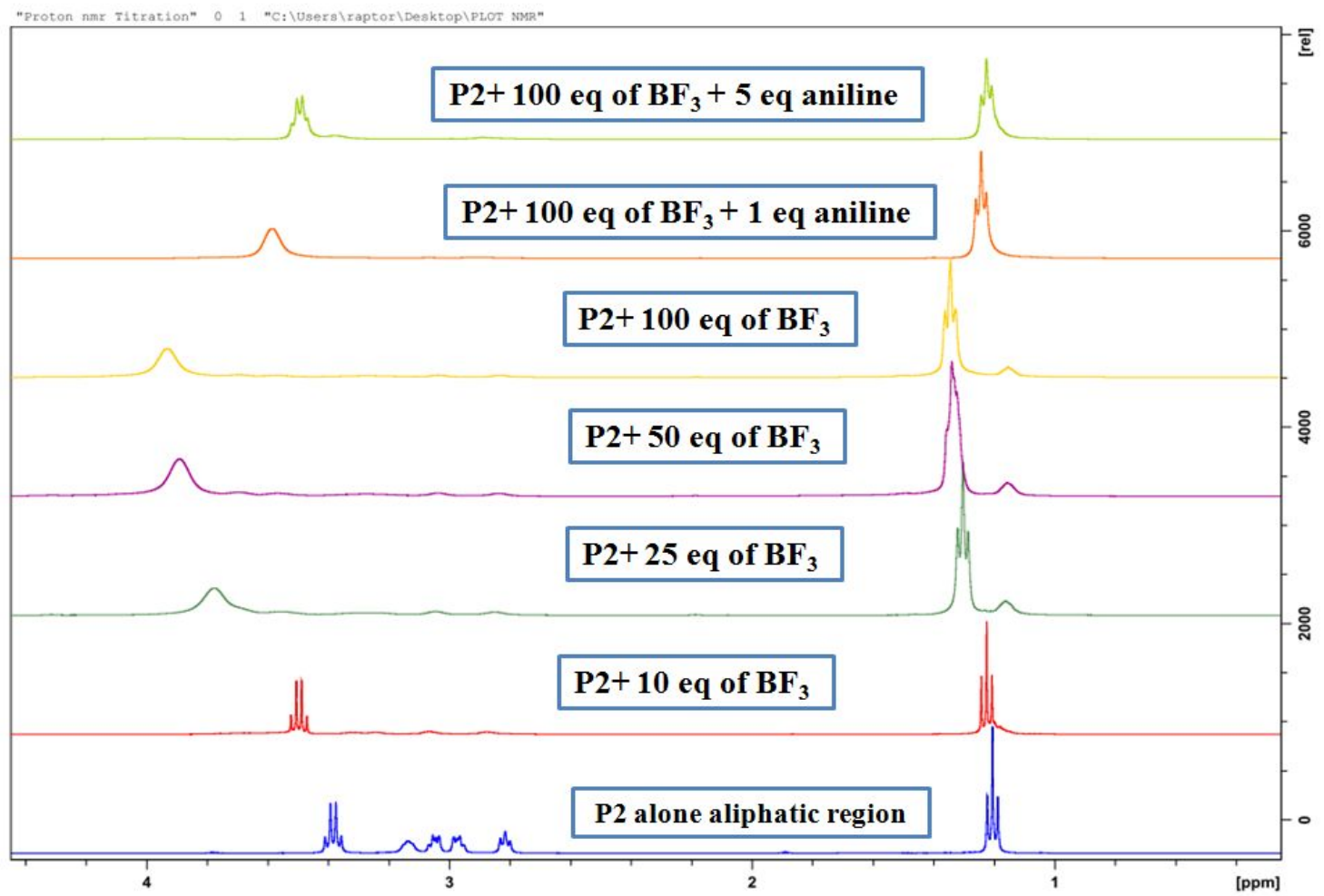

(b) 
Figure S6. Emission spectra of $\mathbf{P 2}$ and normalized emission spectra of PBC2 $(20 \mu \mathrm{M})$ recorded in various solvents.

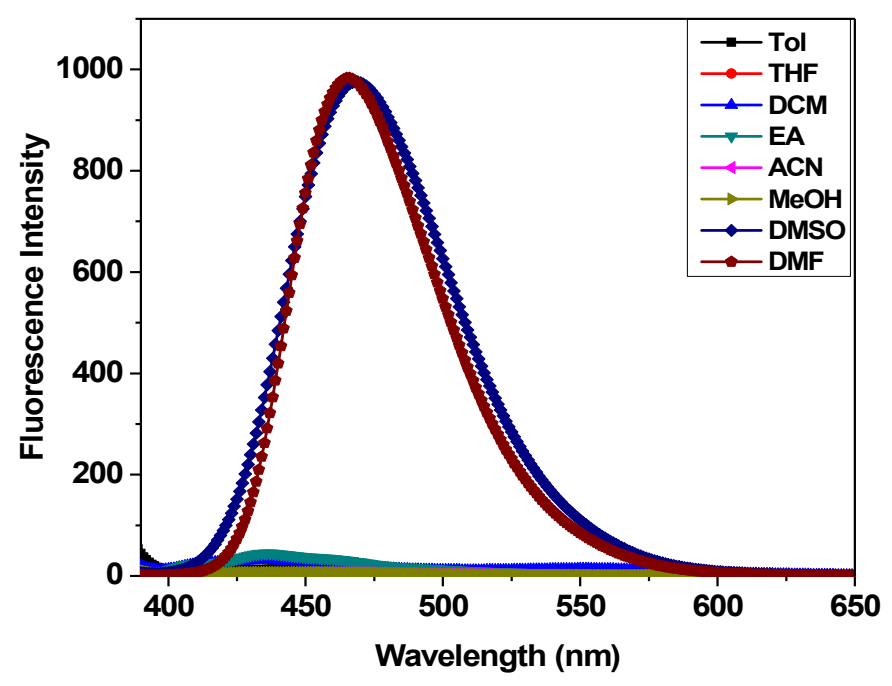

(a)

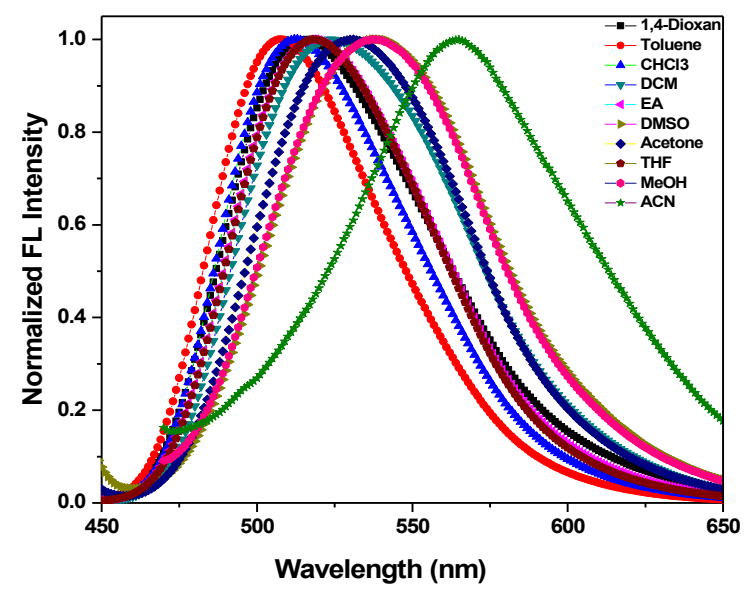

(b)

"The emission spectra of PBC2 in solvents with low, medium and high polarity were studied and the corresponding normalized spectra are shown in Figure S6b. It can be seen that the emission intensity wavelength of PBC2 was bathochromically shifted from toluene to ACN. This result indicates that the bathochromic shift character in polar solvents of PBC2 was attributed to the TICT nature of PBC2 complex." 
Figure S7. Emission spectra of $\mathbf{P 2}(20 \mu \mathrm{M})$ recorded with increasing the DMSO content in THF solvent.

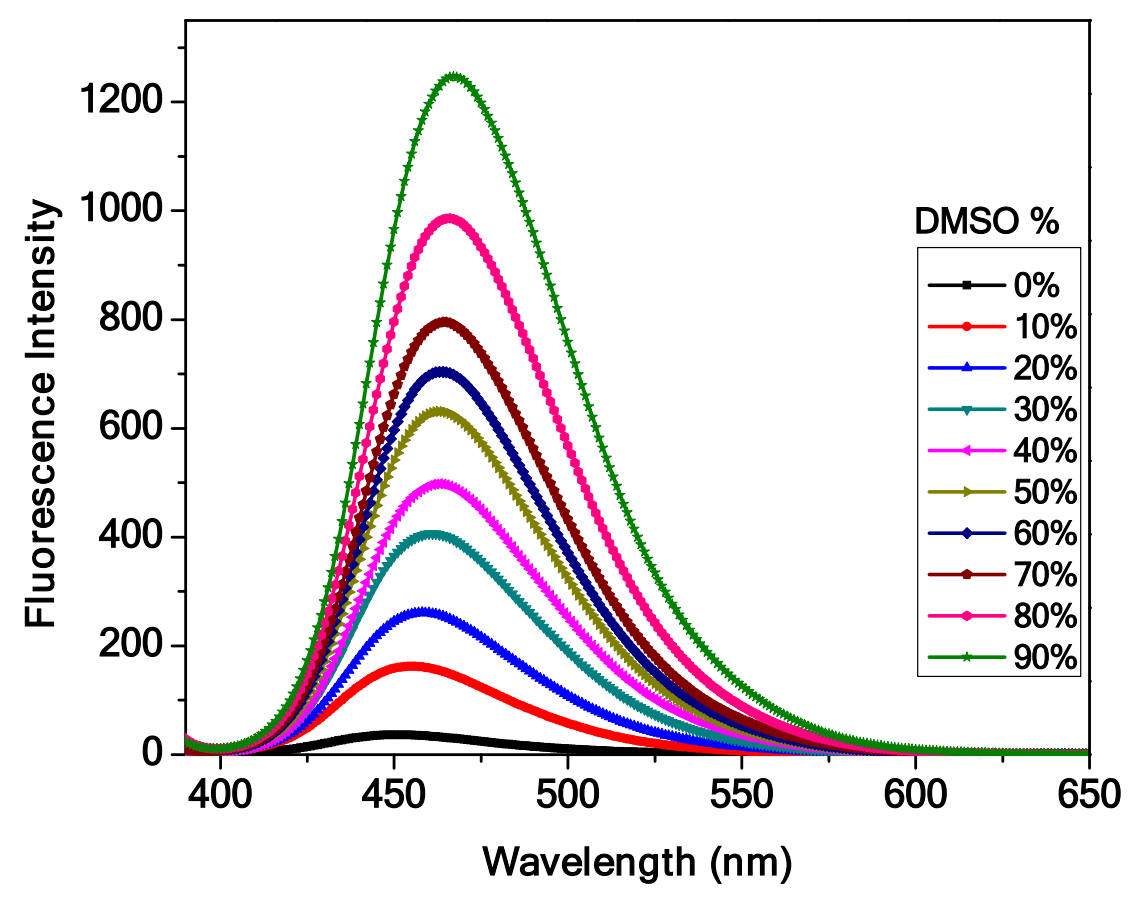


Figure S8. The fluorescence decay profiles of P2, PC2 and PBC2.

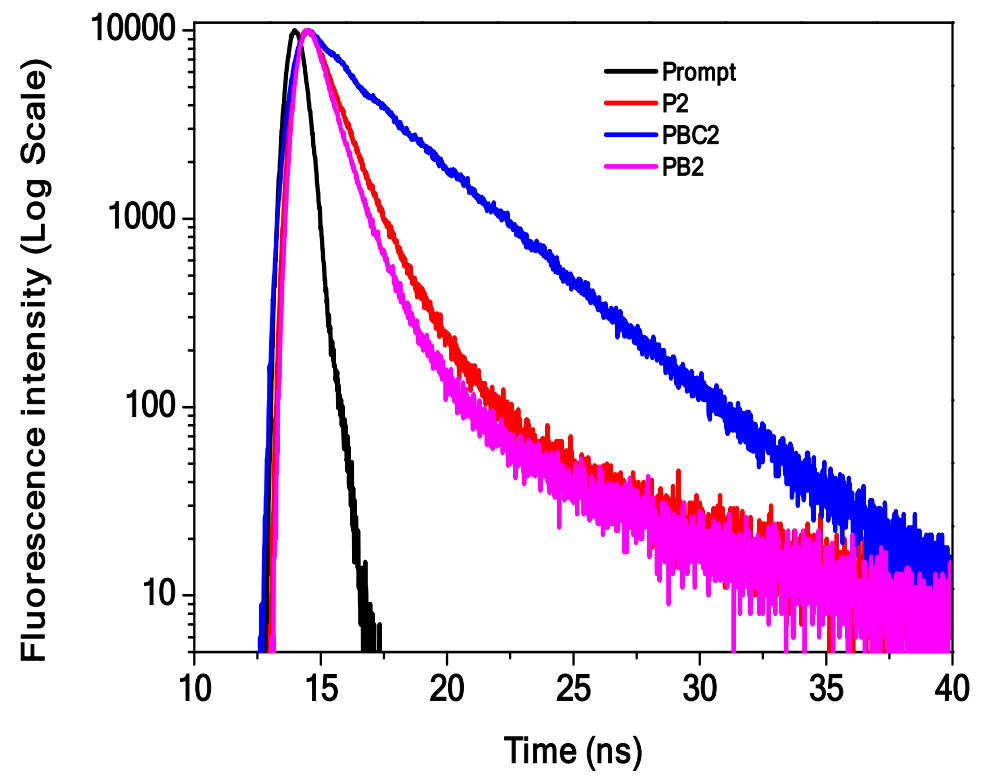


Figure S9. (a) Changes in the absorbance (b) Changes in the fluorescence intensities of PB2 (20 $\mu \mathrm{M})$ after the addition of different organic functional groups (1000 equiv.) and the excitation wavelength is $405 \mathrm{~nm}$.
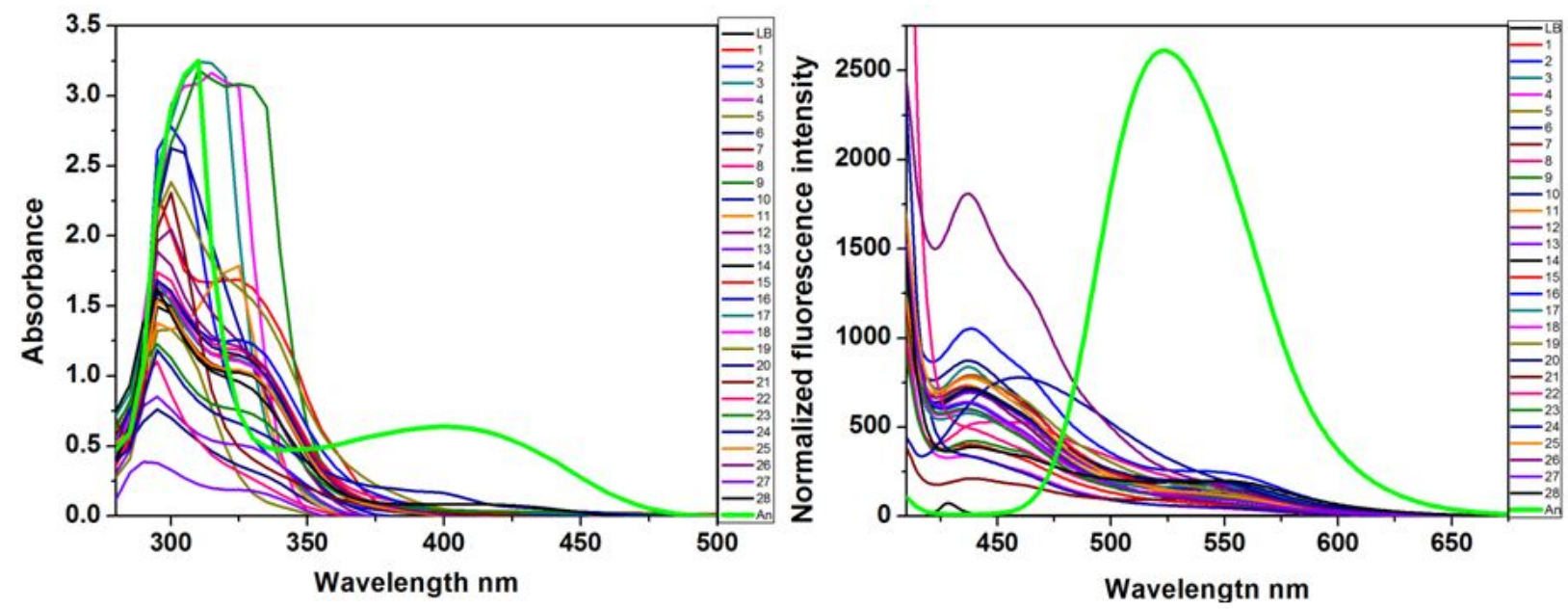
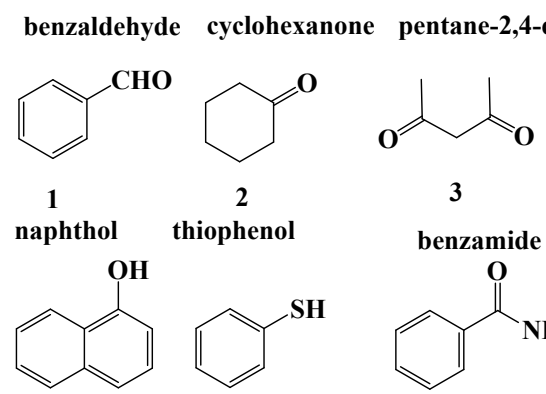

9

benzoic acid<smiles>O=C(O)c1ccccc1</smiles>

17<smiles>O=C[C@H](O)[C@@H](O)[C@H](O)[C@H](O)CO</smiles>

24<smiles>Sc1ccccc1</smiles>

10
3<smiles>NC(=O)c1ccccc1</smiles>

11<smiles>COC(=O)CC(C)=O</smiles>

4 nitrobenzene<smiles>O=[N+]([O-])c1ccccc1</smiles><smiles>CCCCCCCCCCCCCCCCCC(=O)c1ccccc1</smiles>

18 phenylacetylene<smiles>C#Cc1ccccc1</smiles><smiles>Cc1ccc(Cl)cc1</smiles>

19 styrene

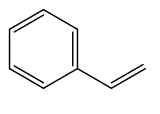

26
12

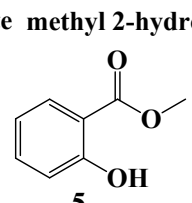

acetonitrile acrylonitrile<smiles>CCCC#N</smiles>

13 hydrazine phenylhydrazine

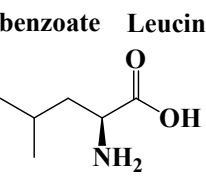

6 7 malononitrile $\mathbf{N} \mathbb{N}^{\mathbf{N}}$

15<smiles>NNc1ccccc1</smiles>
pyrrolidinone<smiles>O=C1CCCN1</smiles>

28<smiles>NNC(N)=S</smiles>

22 aniline<smiles>Nc1ccccc1</smiles>

29
t-Butanol Phenol<smiles>CC(C)(O)c1ccccc1</smiles>

$\begin{array}{ll}7 & 8\end{array}$

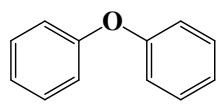

16<smiles>c1ccncc1</smiles>

27 
Figure S10. (a) Changes in the emission spectra of P2 $(20 \mu \mathrm{M})$ after adding the mixture of $\mathrm{BF}_{3} . \mathrm{OEt}_{2}$ and different organic functional groups (10 equiv). The excitation wavelength is 405 $\mathrm{nm}$.

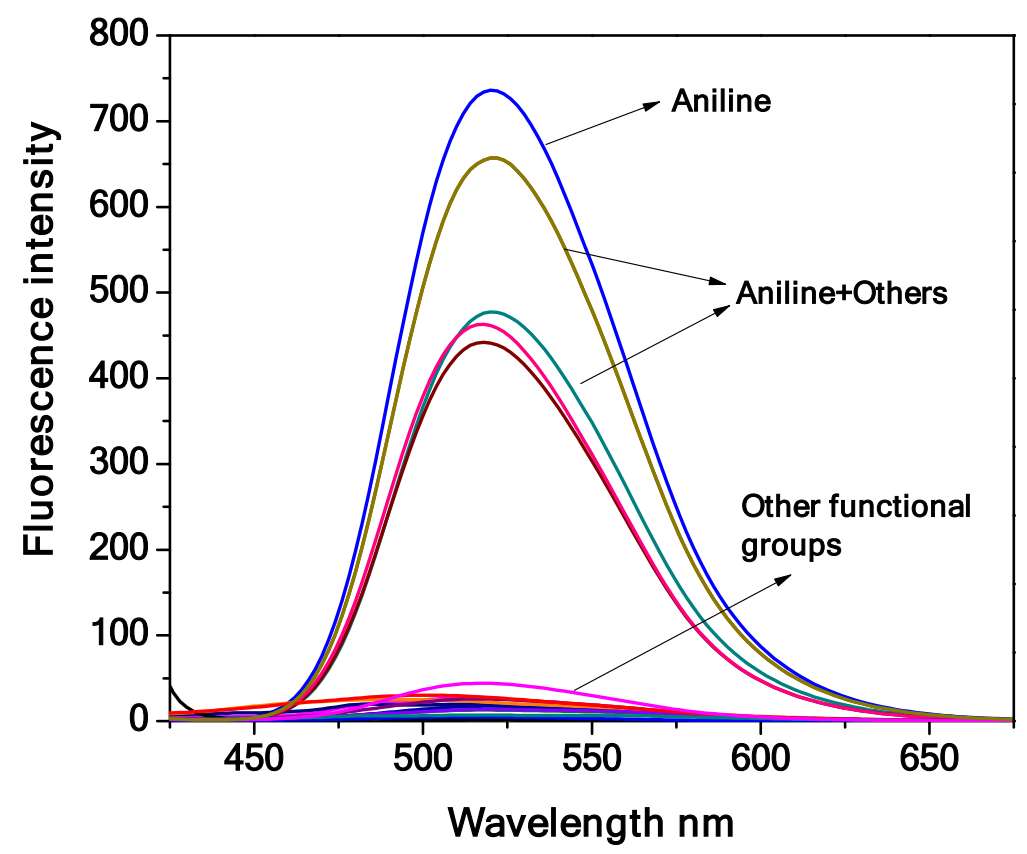


Figure S11. Selectivity studies recorded using emission spectra by adding (a) various boron compounds and (b) mixture of aniline (An)+boron compounds.

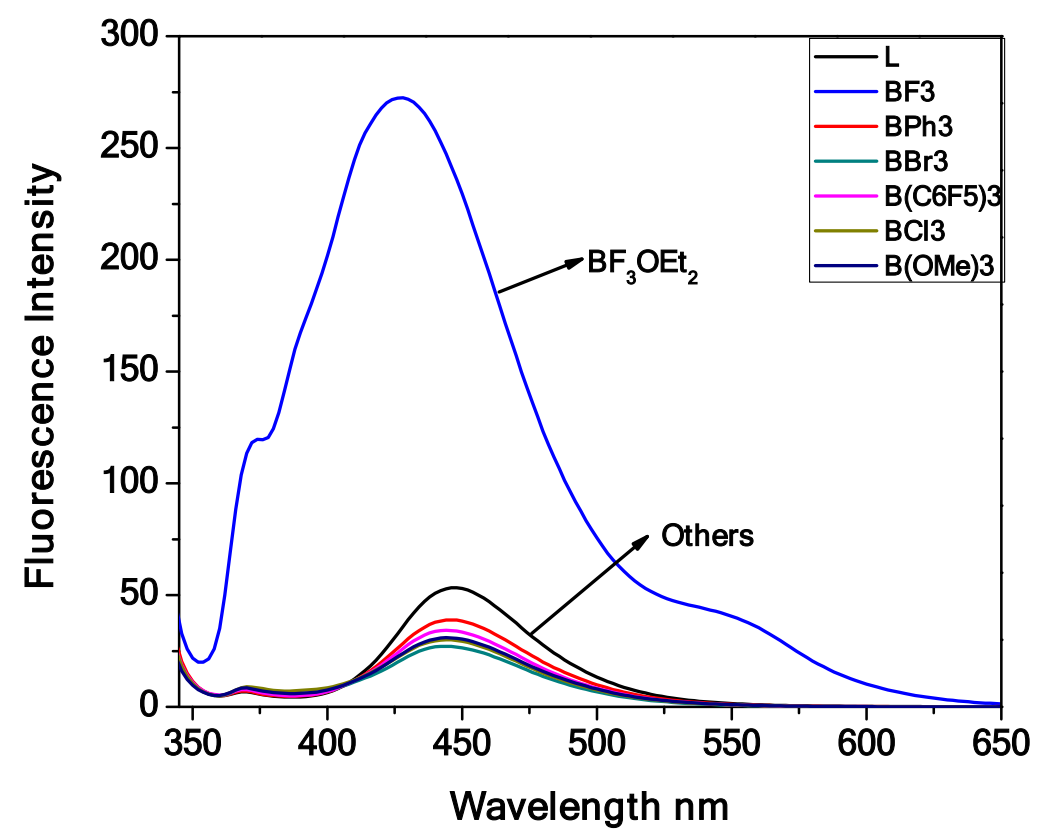

(a)

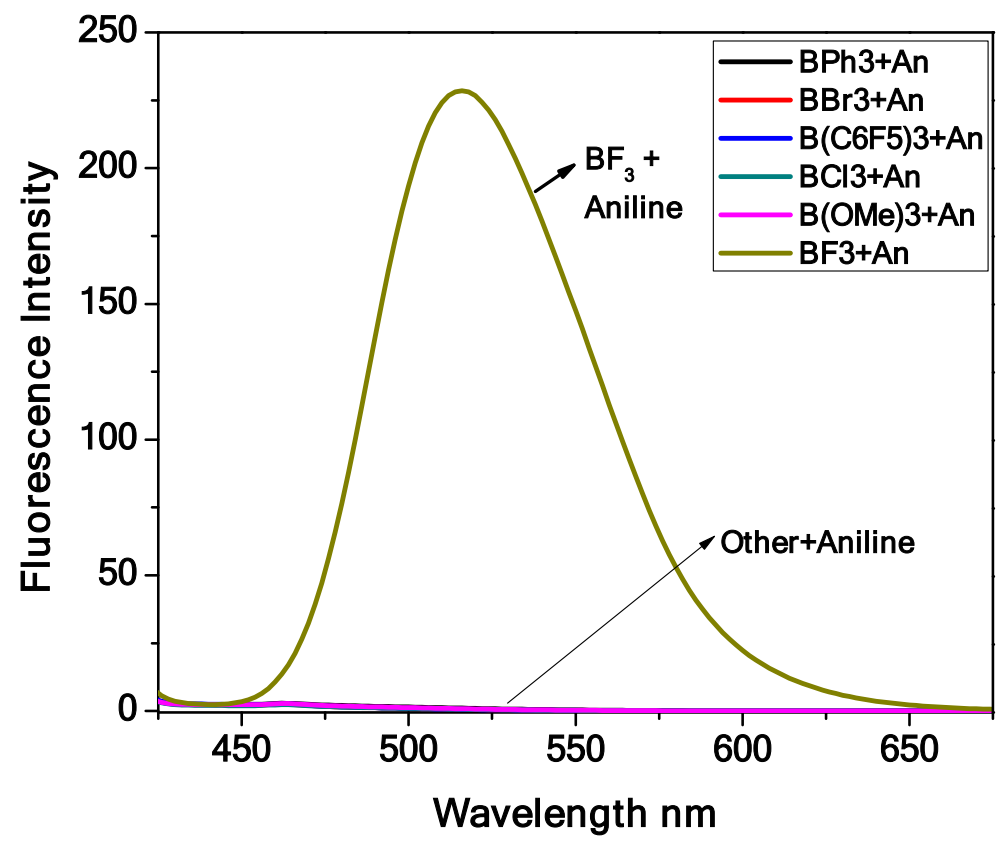

(b) 
Figure S12. Emission titrations of PB2 $\left(20 \mu \mathrm{M}\right.$ of $\mathbf{P 2}$ containing 800 equiv. of $\mathrm{BF}_{3}$. $\left.\mathrm{OEt}_{2}\right)$ performed with the addition of trimethylamine (TEA).

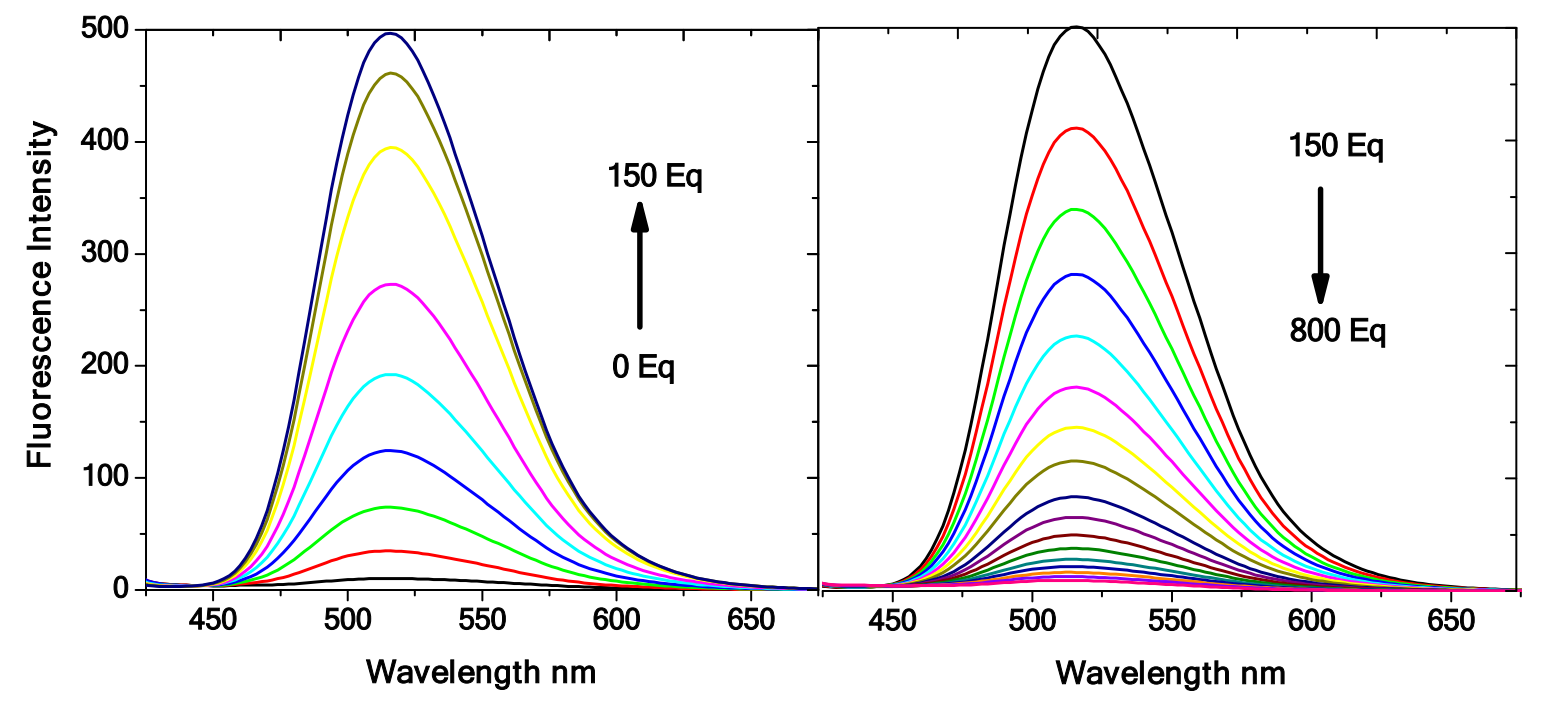

Figure S13. ORTEP structure of P2-BF4 adducts.

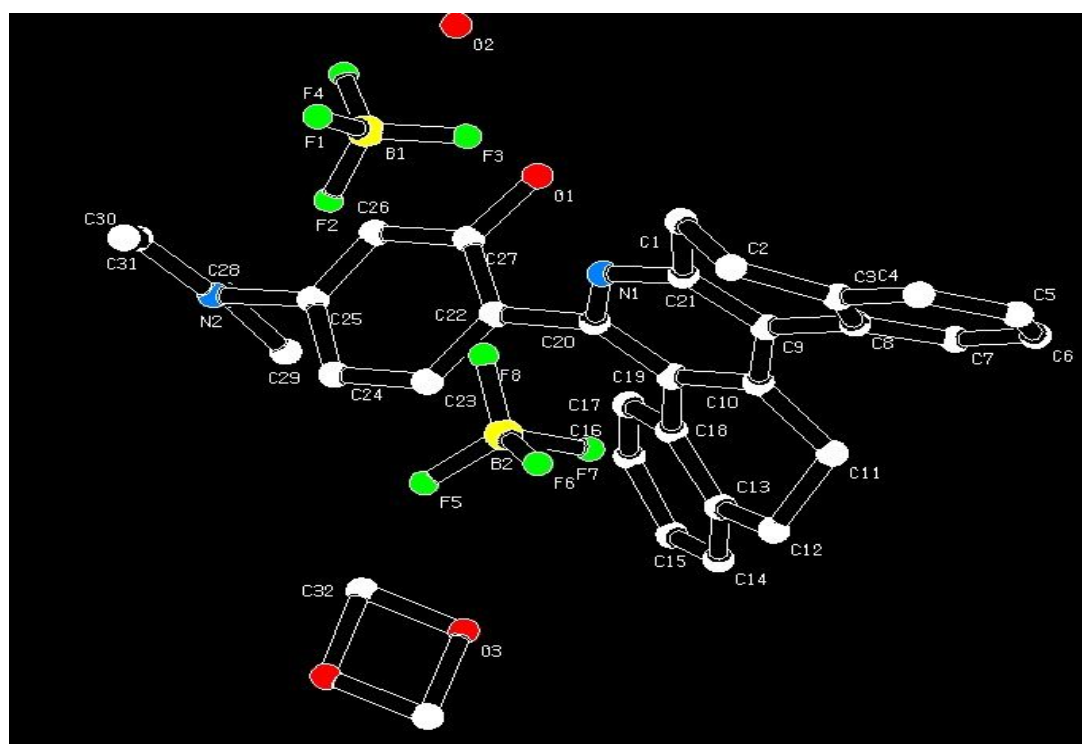


Figure S14. Changes observed in the fluorescence intensities of P2 $(20 \mu \mathrm{M})$ as a function of $\left[\right.$ Aniline- $\left.\mathrm{BF}_{3} \cdot \mathrm{OEt}_{2}\right]\left(1 \times 10^{-3} \mathrm{M}\right)$ at $516 \mathrm{~nm}$.

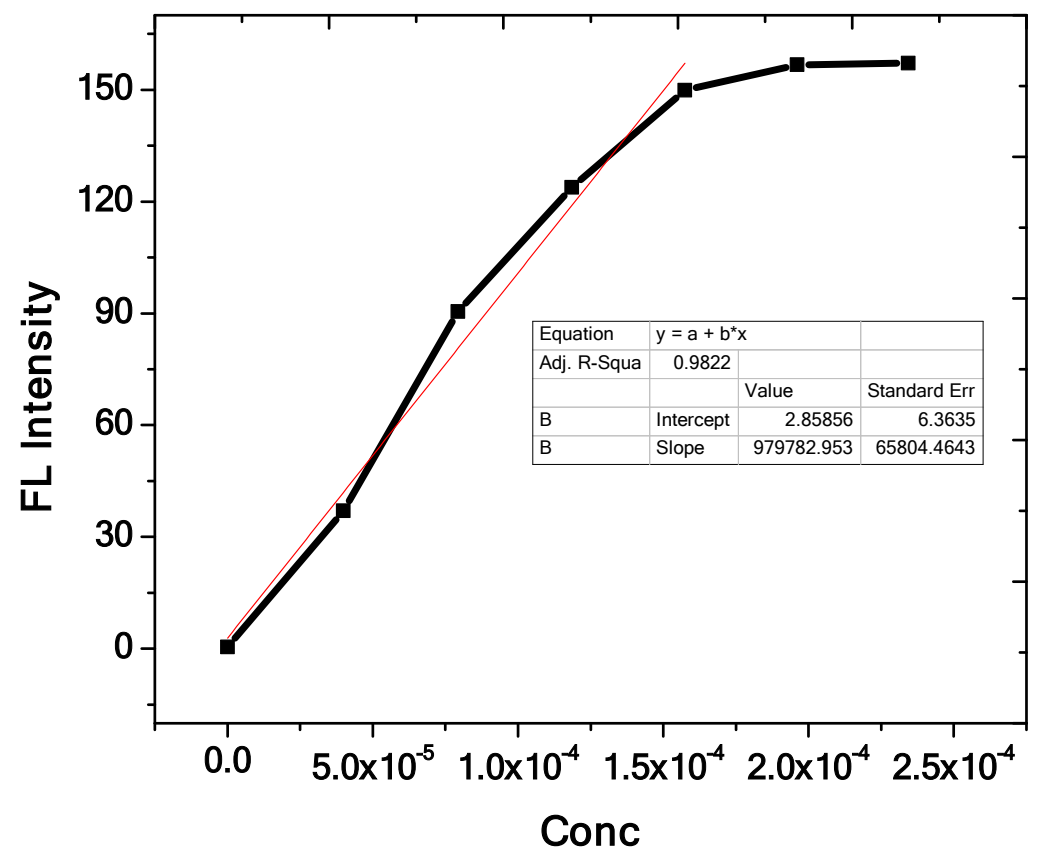

Figure S15. Changes observed in the fluorescence intensities of P2 $(20 \mu \mathrm{M})$ as a function of [4Aminobenzoic acid-BF $\left.3 . \mathrm{OEt}_{2}\right]\left(1 \times 10^{-3} \mathrm{M}\right)$ at $516 \mathrm{~nm}$.

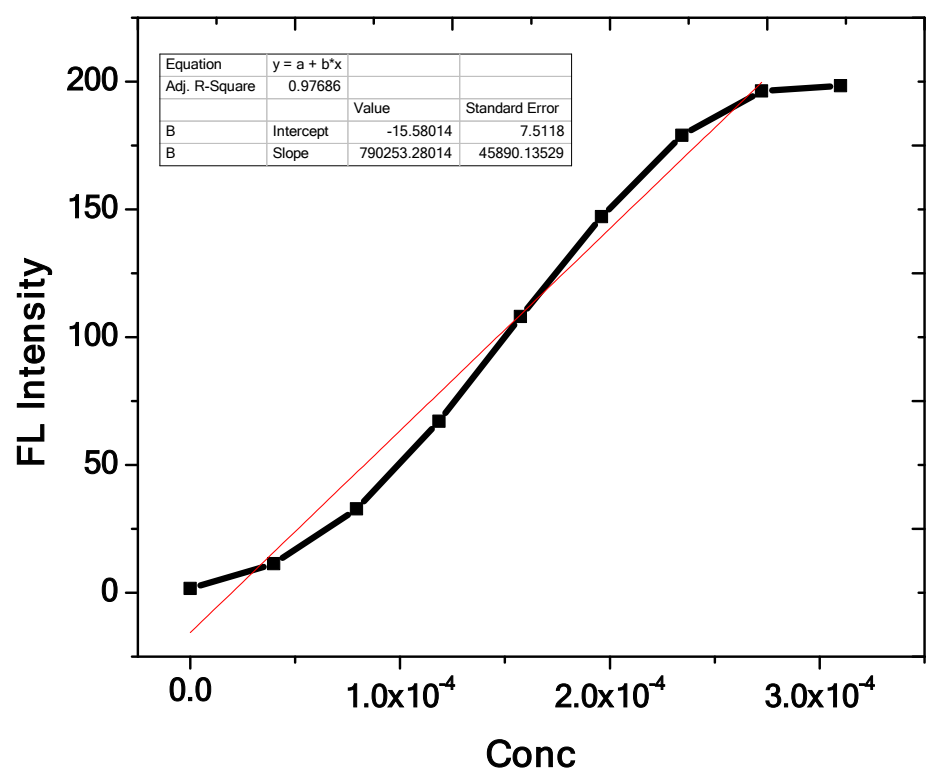


Figure S16. Changes observed in the fluorescence intensities of $\mathbf{P 2}(20 \mu \mathrm{M})$ as a function of [4Methoxyaniline- $\left.\mathrm{BF}_{3} \cdot \mathrm{OEt}_{2}\right]\left(1 \times 10^{-3} \mathrm{M}\right)$ at $516 \mathrm{~nm}$.

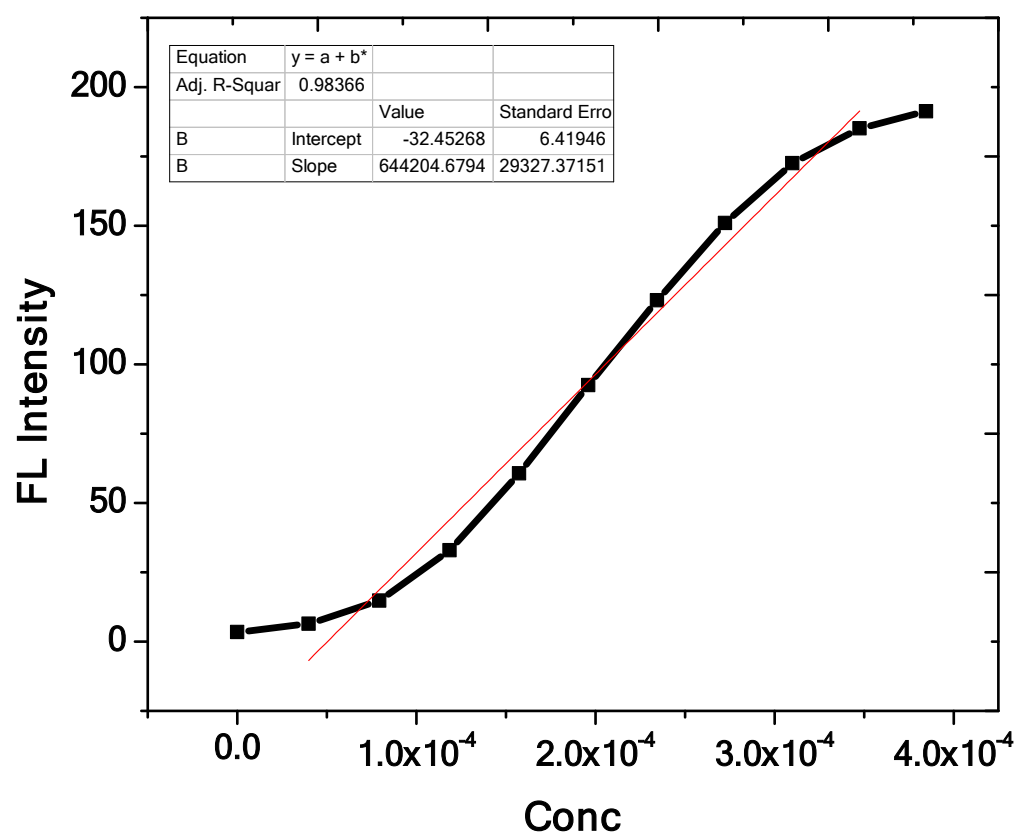

Figure S17. Changes observed in the fluorescence intensities of P2 $(20 \mu \mathrm{M})$ as a function of [2aminopyridine- $\left.\mathrm{BF}_{3} . \mathrm{OEt}_{2}\right]\left(1 \times 10^{-3} \mathrm{M}\right)$ at $516 \mathrm{~nm}$.

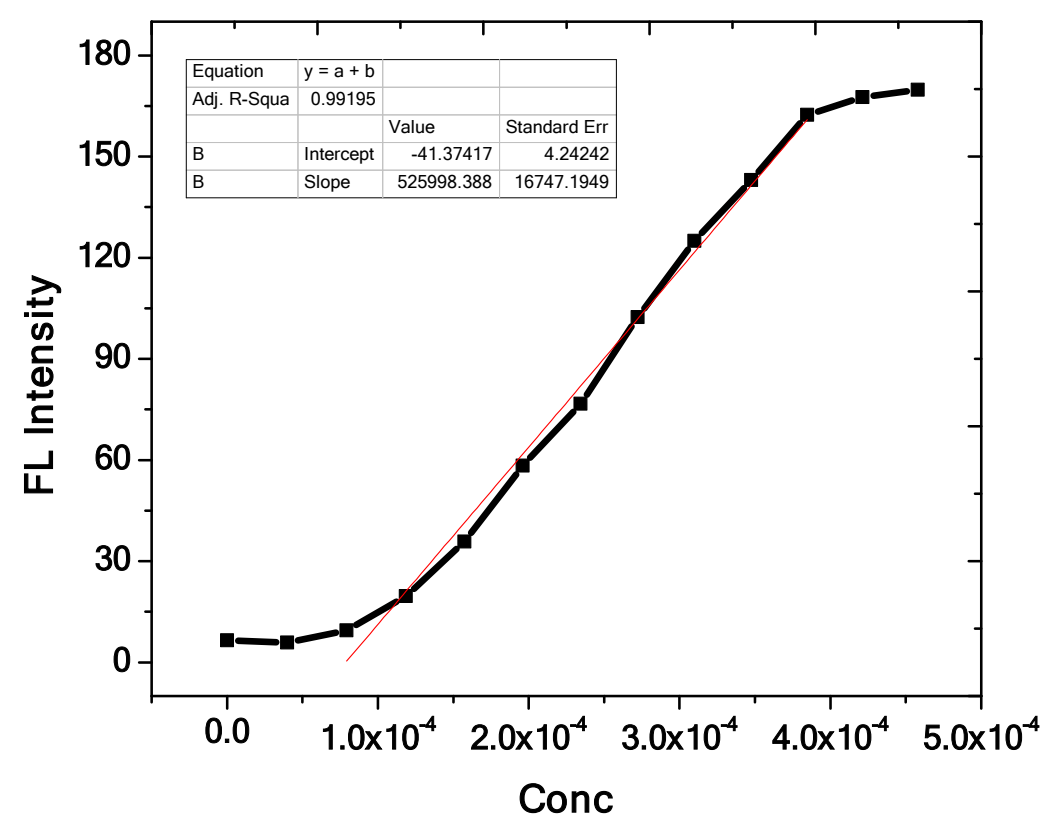


Figure S18. Changes observed in the fluorescence intensities of P2 $(20 \mu \mathrm{M})$ as a function of [1napthylamine- $\left.\mathrm{BF}_{3} \cdot \mathrm{OEt}_{2}\right]\left(1 \times 10^{-3} \mathrm{M}\right)$ at $516 \mathrm{~nm}$.

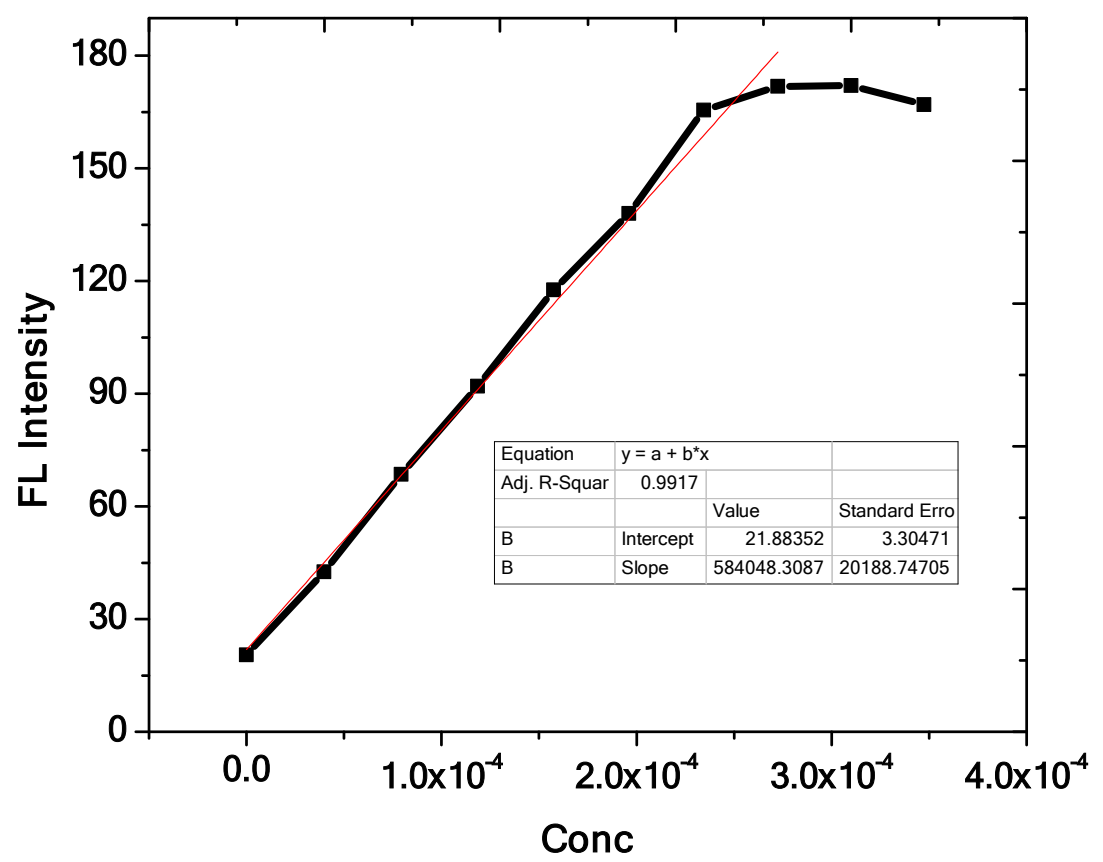

Figure S19. Changes observed in the fluorescence intensities of P2 $(20 \mu \mathrm{M})$ as a function of $\left[4,4^{\prime}\right.$-diaminodiphenylmethane- $\left.\mathrm{BF}_{3} \cdot \mathrm{OEt}_{2}\right]\left(1 \times 10^{-3} \mathrm{M}\right)$ at $516 \mathrm{~nm}$.

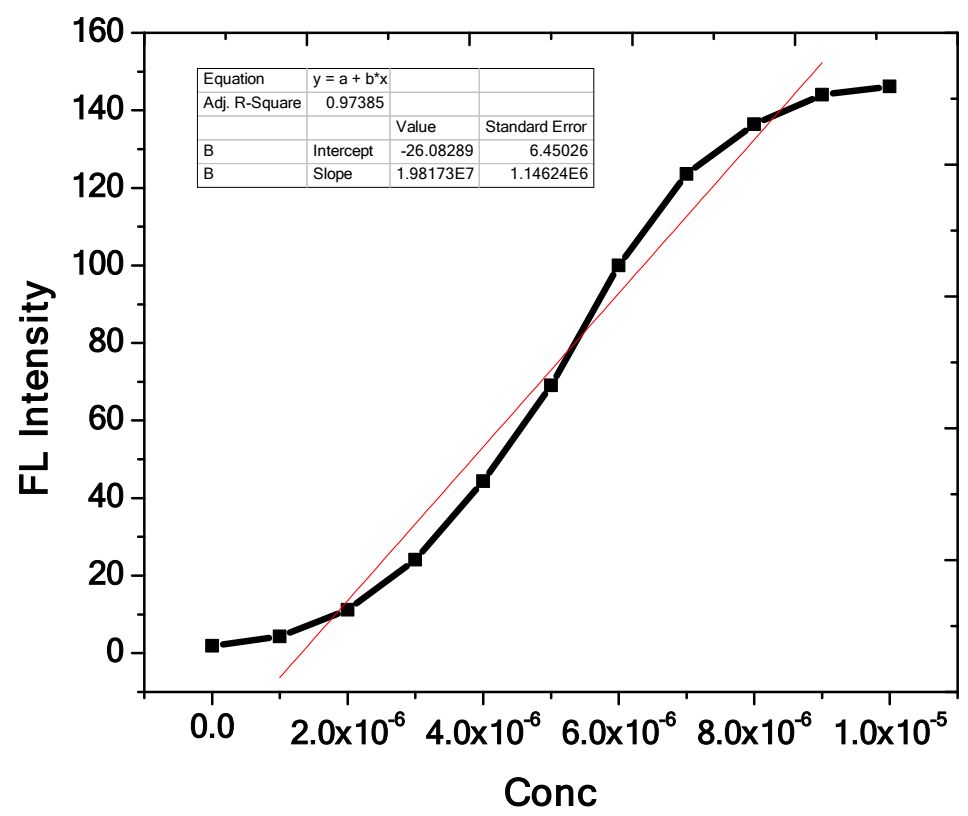


Figure S20. Jobs plot according to the method of continuous variation, indicating the 1:1 stoichiometry of the $\mathbf{P 2}-\mathrm{BF}_{3}$ complex.

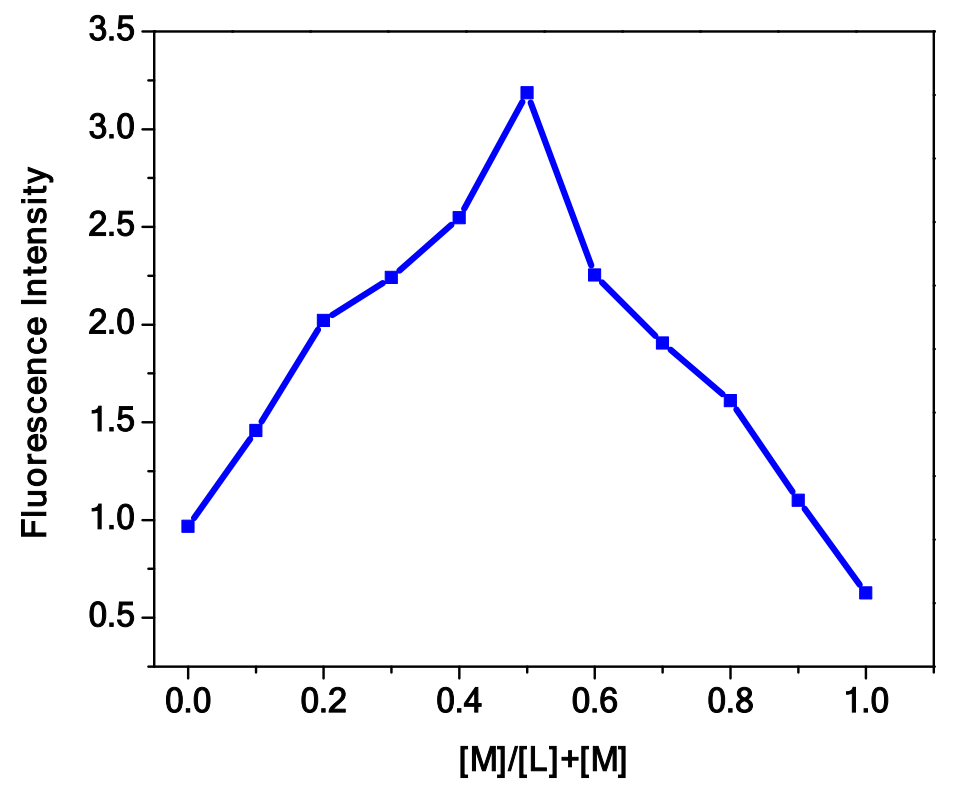

Figure S21. Emission titrations of $\mathbf{P 2}(20 \mu \mathrm{M})$ performed with the addition of $1 \times 10^{-3} \mathrm{M}$ concentration of $\mathrm{TEA}+\mathrm{BF}_{3} . \mathrm{OEt}_{2}$ mixture.

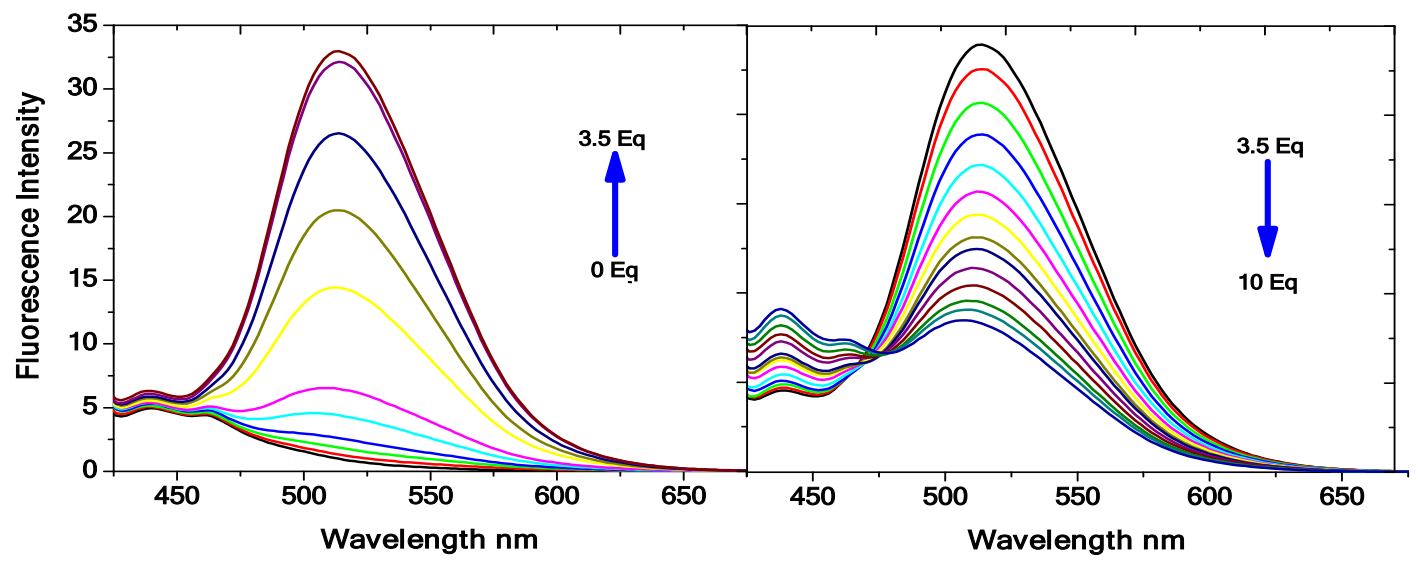


Figure S22. Time dependent emission titrations of $\mathbf{P 2}(20 \mu \mathrm{M})$ performed with the addition of 10 equiv. of $1 \times 10^{-3} \mathrm{M}$ concentration of 4-aminobenzoic acid $+\mathrm{BF}_{3} \cdot \mathrm{OEt}_{2}$ mixture.

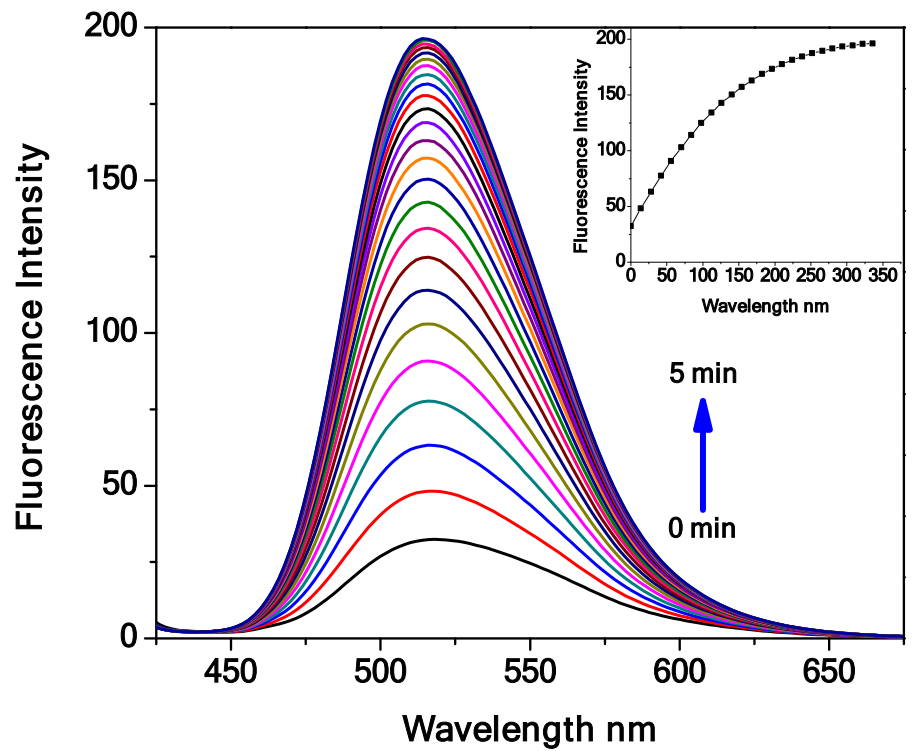

Figure S23. Time dependent emission titrations of P2 $(20 \mu \mathrm{M})$ performed with addition of 10 equiv. of $1 \times 10^{-3} \mathrm{M}$ concentration of 4-methoxy aniline $+\mathrm{BF}_{3} \cdot \mathrm{OEt}_{2}$ mixture.

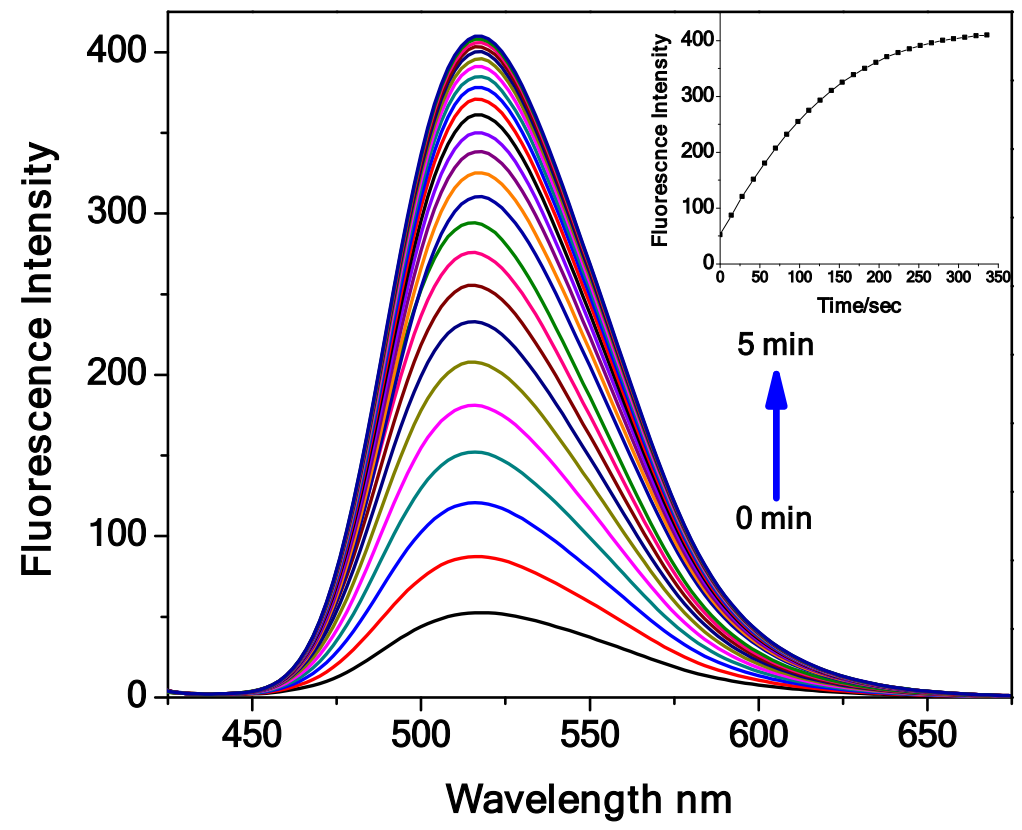


Figure S24. Time dependent emission titrations of P2 $(20 \mu \mathrm{M})$ performed with the addition of 10 equiv. of $1 \times 10^{-3} \mathrm{M}$ concentration of 1-napthylamine $+\mathrm{BF}_{3} . \mathrm{OEt}_{2}$ mixture.

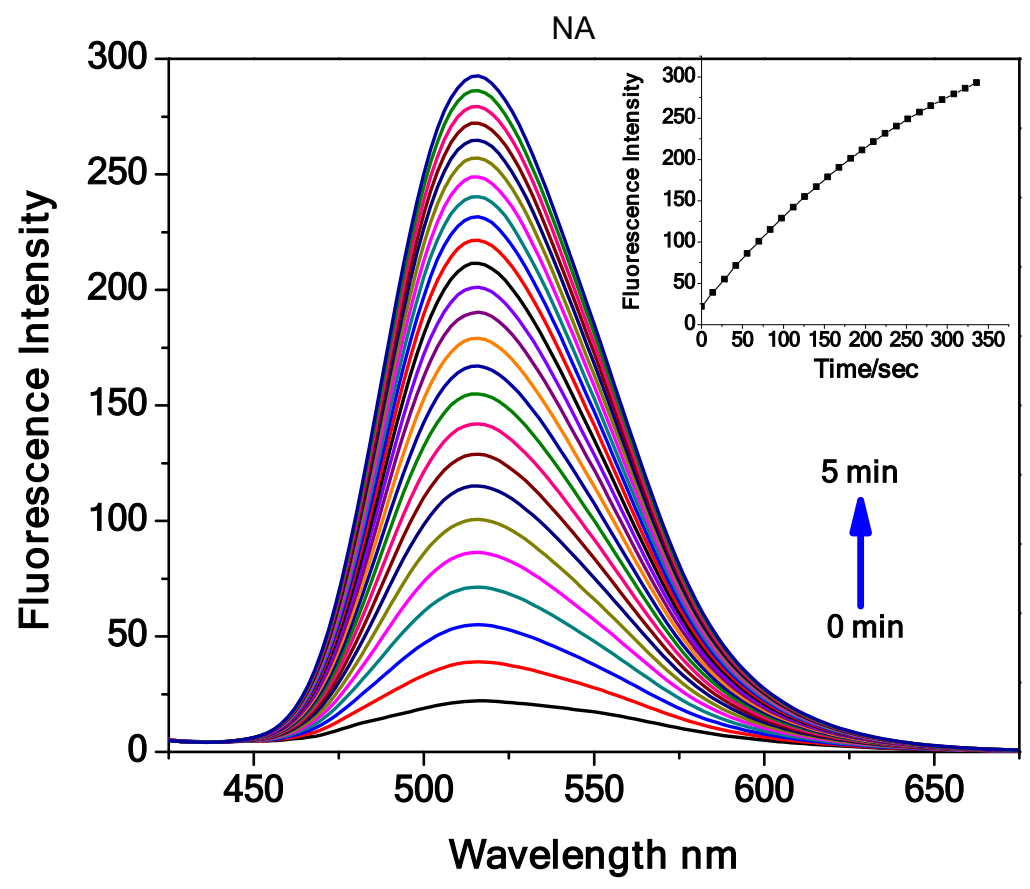

Figure S25. Time dependent emission titrations of $\mathbf{P 2}(20 \mu \mathrm{M})$ performed with addition of 10 equiv. of $1 \times 10^{-3} \mathrm{M}$ concentration of 2-aminopyridine $+\mathrm{BF}_{3} . \mathrm{OEt}_{2}$ mixture.

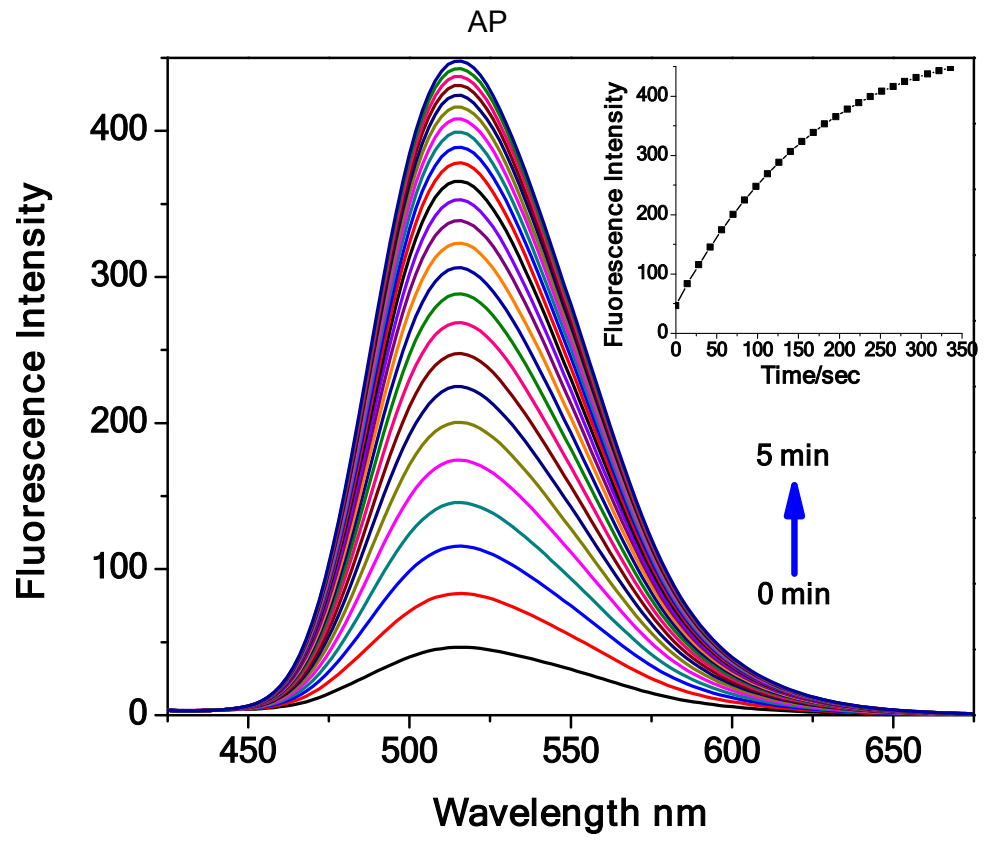


Figure S26. Time dependent emission titrations of P2 $(20 \mu \mathrm{M})$ performed with the addition of 10 equiv. of $1 \times 10^{-3} \mathrm{M}$ concentration of 4,4'-diaminodiphenylmethane $+\mathrm{BF}_{3} . \mathrm{OEt}_{2}$ mixture.

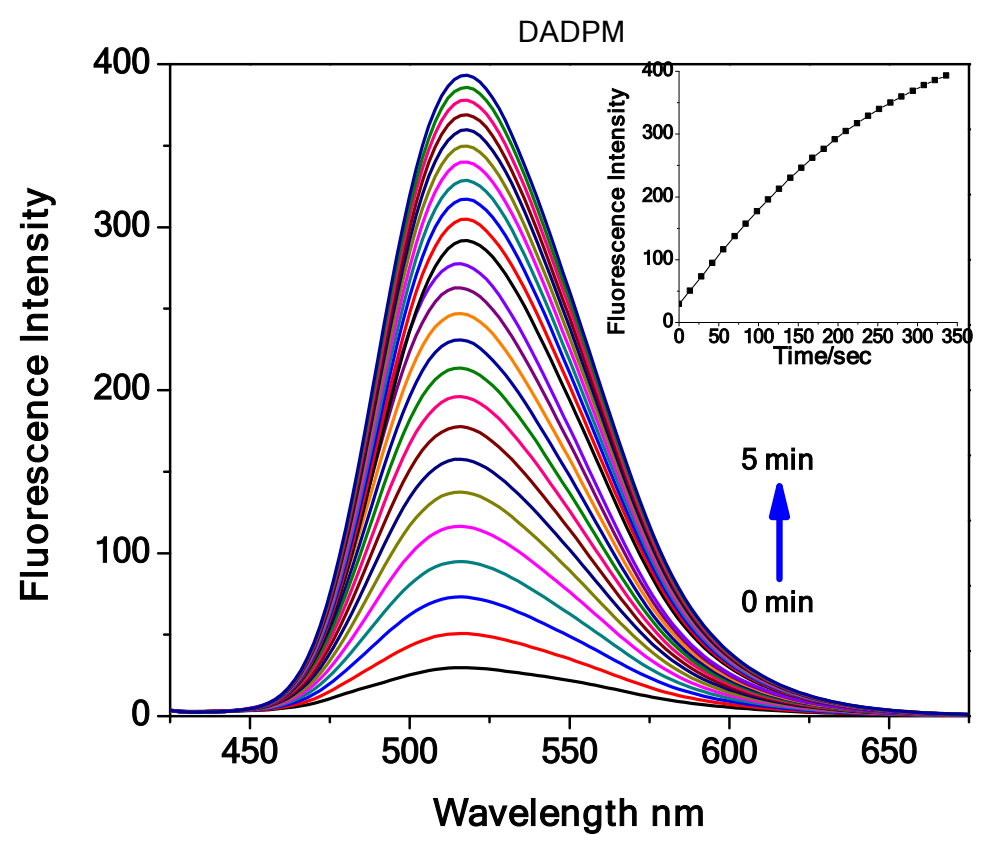

Figure S27. Time dependent emission titrations of P2 $(20 \mu \mathrm{M})$ performed with the addition of 10 equiv. of $1 \times 10^{-3} \mathrm{M}$ concentration of diethylamine $+\mathrm{BF}_{3} \cdot \mathrm{OEt}_{2}$ mixture.

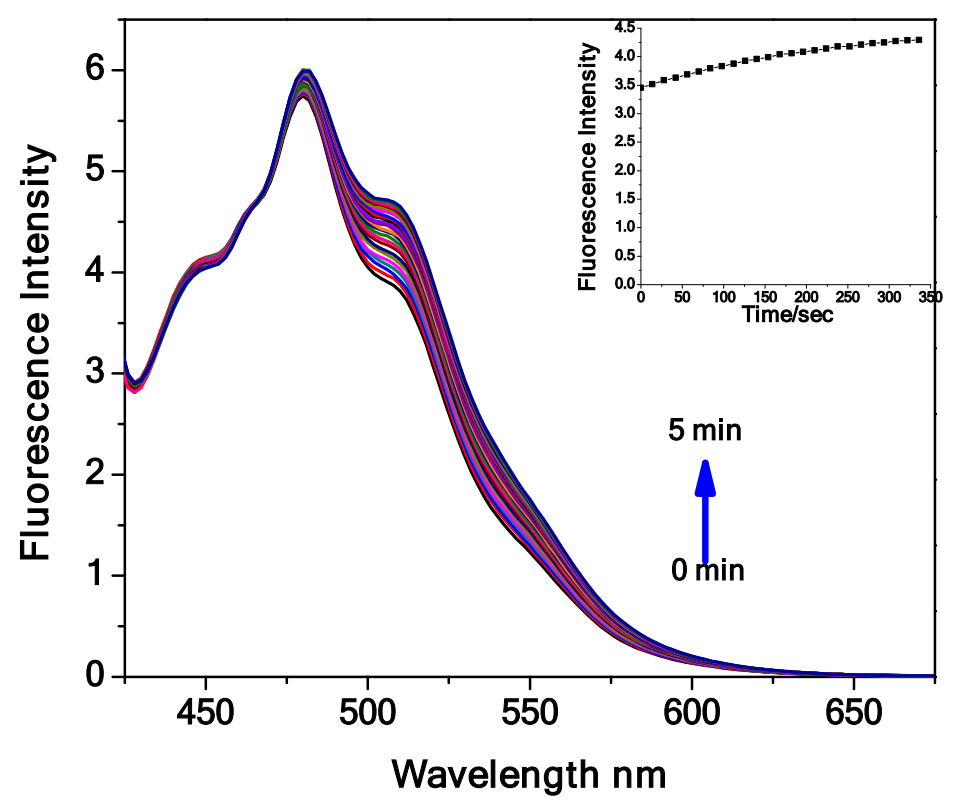


Figure S28. Time dependent emission titrations of $\mathrm{BF}_{3} \cdot \mathrm{OEt}_{2}(20 \mu \mathrm{M})$ performed with the addition of 10 equiv. of $1 \times 10^{-3} \mathrm{M}$ concentration of ethylamine $+\mathrm{BF}_{3} \cdot \mathrm{OEt}_{2}$ mixture.

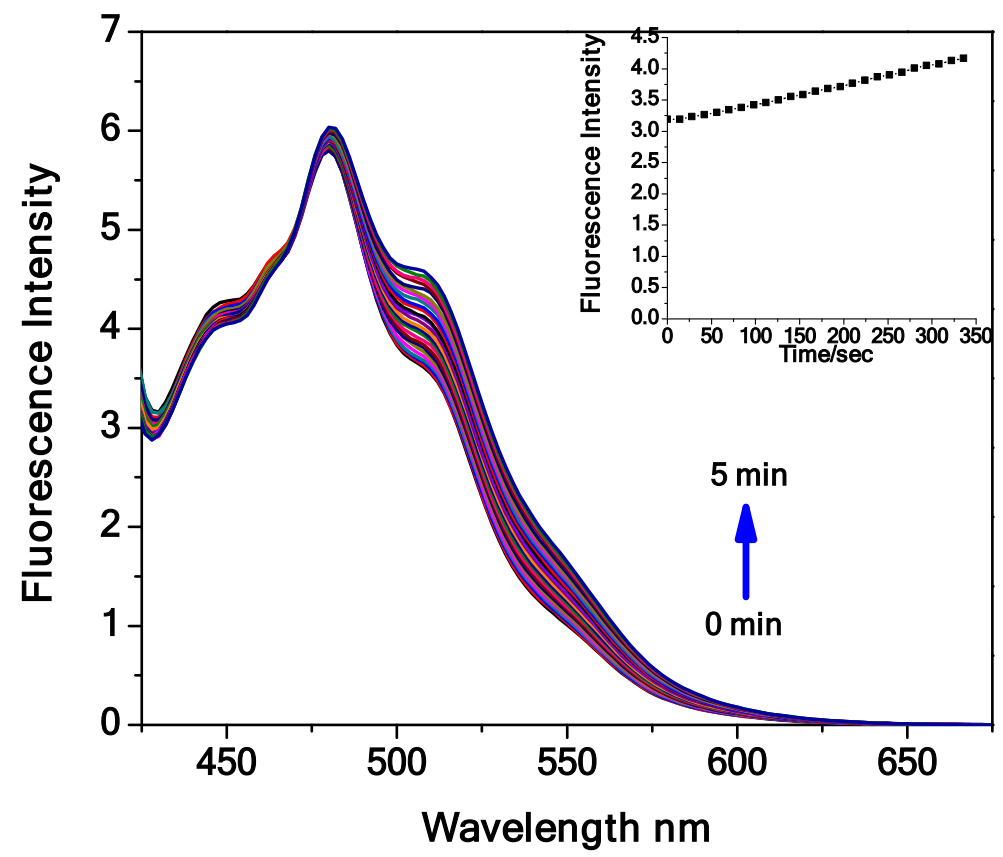

Figure S29. The plot of reaction time as a function of emission intensity given in bar diagram

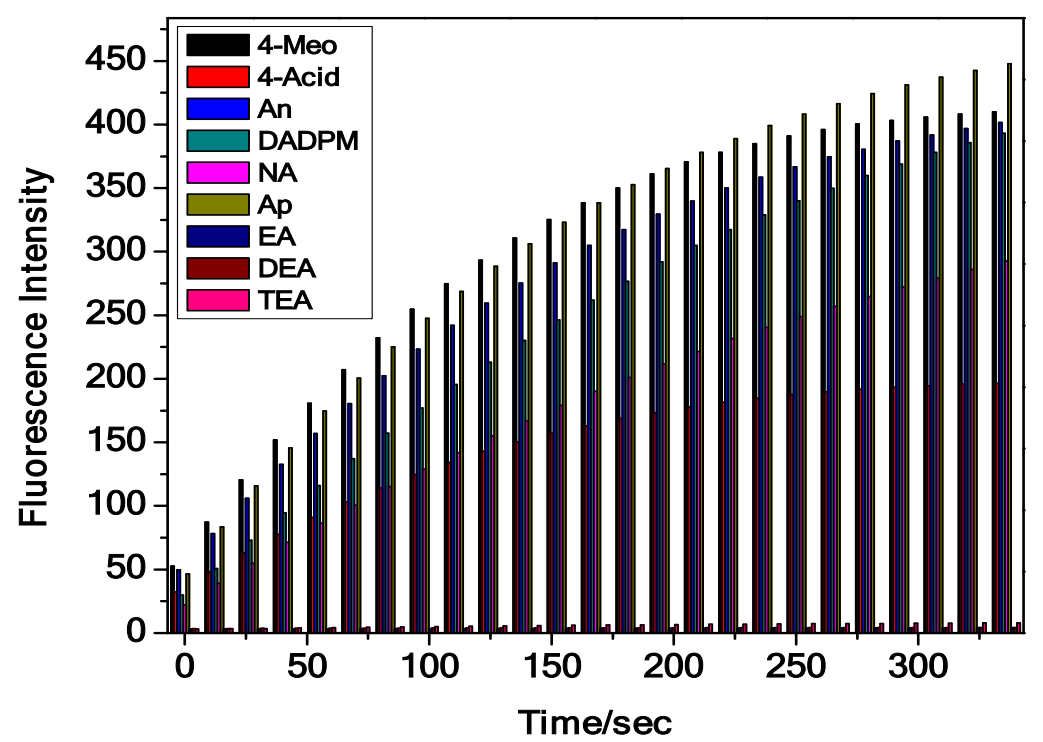


Figure S30. The emission response of $\mathbf{P 2}$ as a function of various amines mixtures

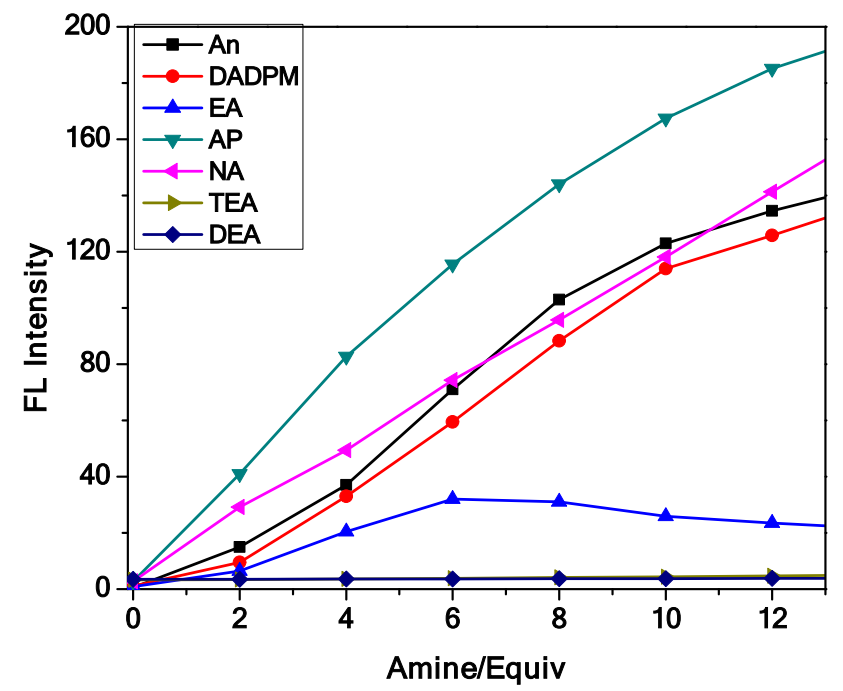

Figure S31. Changes in the emission intensities of P2 $(20 \mu \mathrm{M})$ after adding a mixture of $\mathrm{BF}_{3} . \mathrm{OEt}_{2}$ and different aromatic primary amines (10 equiv.) and the excitation wavelength is 405 $\mathrm{nm}$

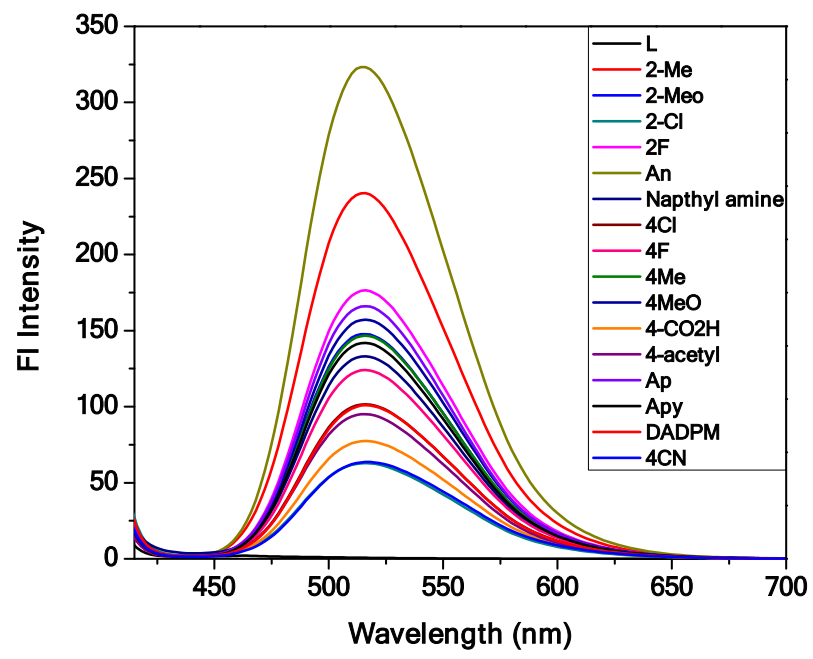


Figure S32. pH sensitivity of sensor $\mathbf{P 2}$.

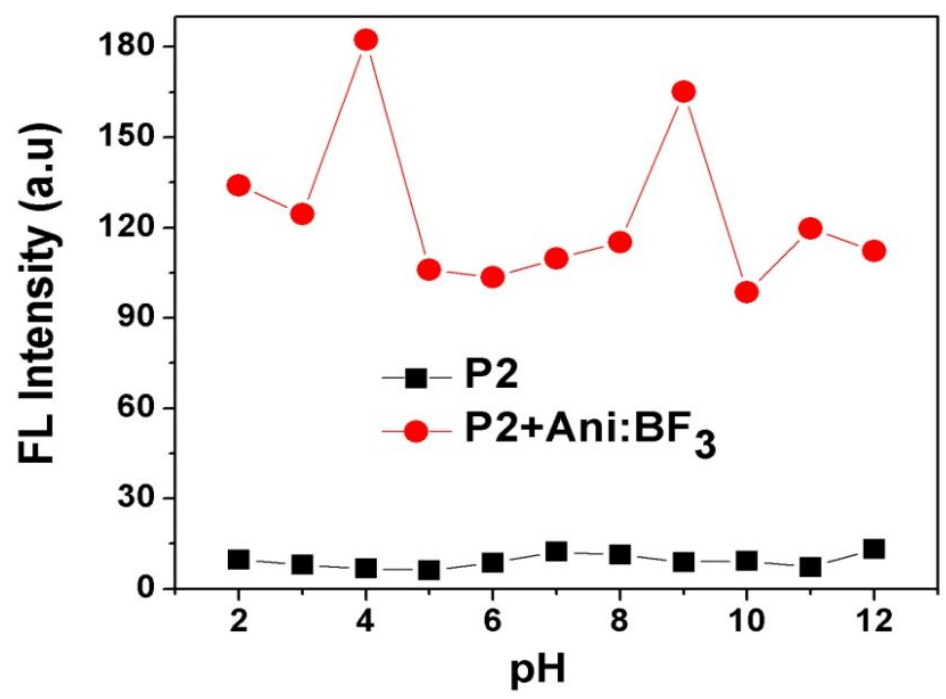

The fluorescence intensity of sensor $\mathbf{P 2}$ in the absence and presence of $\mathrm{Ani}: \mathrm{BF}_{3}$ complex were studied in the $\mathrm{pH}$ range of 2 to 12. As illustrated in Figure S32, the sensor P2 itself show weak emission at $516 \mathrm{~nm}$ and almost no distinct change in emission intensity was observed with the change of $\mathrm{pH}$ range from 2 to 12 . Upon the addition of $\mathrm{Ani}: \mathrm{BF}_{3}$ mixture, the emission intensity was obviously increased over a $\mathrm{pH}$ range of 2 to 12 , illustrating the formation of stable complex PBC2 with sensor $\mathbf{P 2}$ and $\mathrm{Ani}_{\mathrm{B}} \mathrm{BF}_{3}$. Importantly, the detection of aniline is possible wide range of $\mathrm{pH}$ window. These results indicate that $\mathbf{P 2}$ can be used as sensor for aniline over a wide $\mathrm{pH}$ range. 
Figure S33 Fluorescence spectra of receptor $\mathbf{P 2}+\mathrm{Ani}_{\mathrm{B}} \mathrm{BF}_{3}$ mixture $\left(2.0 \mathrm{X} 10^{-5} \mathrm{M}\right)$ in the presence of various metal ions $\left(1 \times 10^{-3} \mathrm{M}\right)$ in water solution (left). The fluorescence intensity at $540 \mathrm{~nm}$ of sensor $\mathrm{P} 2+\mathrm{Ani}: \mathrm{BF}_{3}$ in the presence of various metal ions (right). 1: $\mathrm{Hg}^{2+}$ 2: $\mathrm{Cd}^{2+} 3: \mathrm{Cr}^{2+} 4: \mathrm{Fe}^{3+}$ 5: $\mathrm{Co}^{2+}$ 6: $\mathrm{Ni}^{2+}$ 7: $\mathrm{Sr}^{2+}$ 8: $\mathrm{Fe}^{2+}$ 9: $\mathrm{Mg}^{2+}$ 10: $\mathrm{Na}^{+}$11: $\mathrm{K}^{+}$and 12: $\mathrm{Pb}^{2+}$
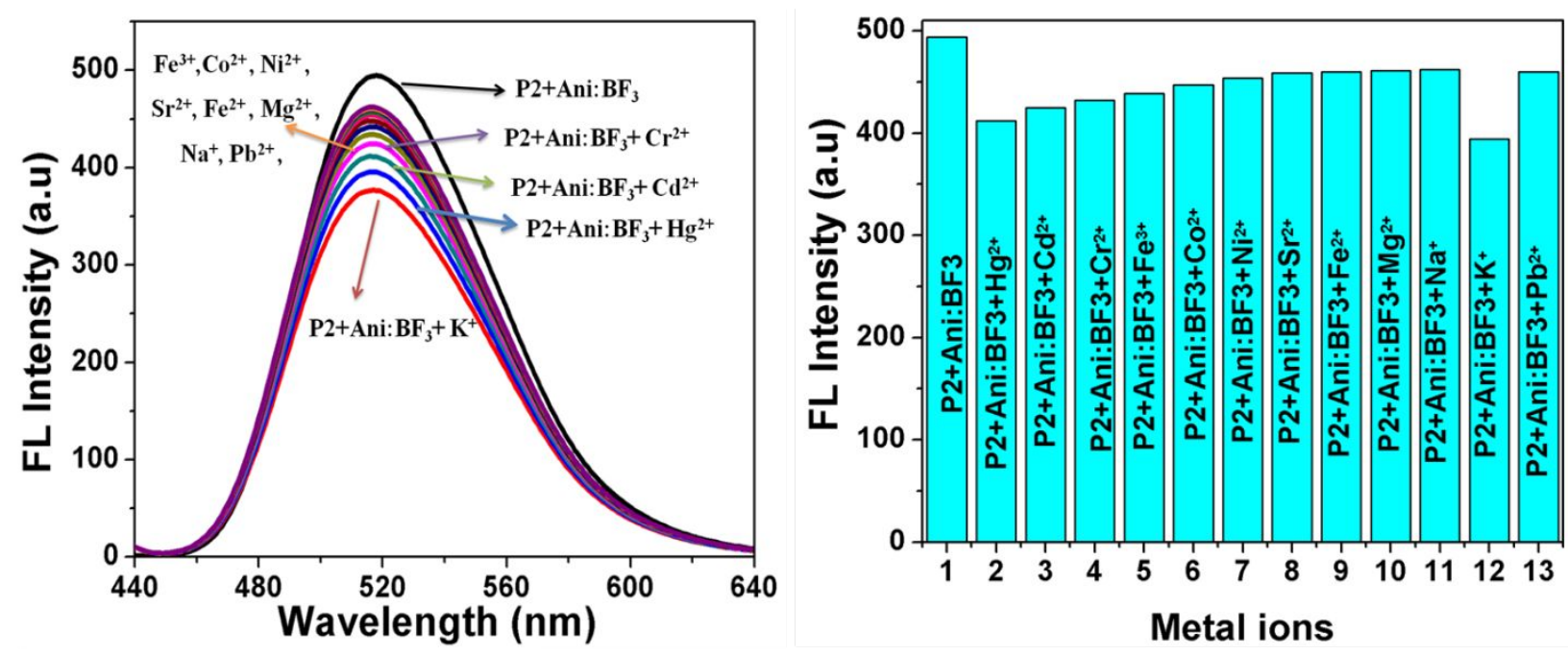

Selectivity study of $\mathbf{P 2}$ in the presence of metal ions, organic acids and electron deficient organic substances:

To study the interference of metal ions, various metal ions including $\mathrm{Fe}^{3+}, \mathrm{Fe}^{2+}, \mathrm{Ni}^{2+}, \mathrm{Sr}^{2+}, \mathrm{Co}^{2+}$, $\mathrm{Mg}^{2+}, \mathrm{Na}^{+}, \mathrm{K}^{+}, \mathrm{Pb}^{2+}, \mathrm{Cr}^{2+}, \mathrm{Cd}^{2+}$, and $\mathrm{Hg}^{2+}$ was added to the sensor $\mathbf{P 2}$ followed by Ani:BF mixture. No significant change in the fluorescent intensity at $516 \mathrm{~nm}\left(2.0 \times 10^{-5} \mathrm{M}\right)$ was found in THF solution, suggesting that the sensor showed good selectivity to aniline in the presence of other metal ions (Figure S33). To further explore the sensing properties of $\mathbf{P 2}$ in the presence of acids, the fluorescence response of $\mathbf{P 2}$ and $\mathrm{Ani}_{\mathrm{BF}}$ mixture $(20 \mu \mathrm{M})$ in THF solution was studied by adding various organic acids (5.0 equiv.). The addition of the following organic acids including 3MPA, AA, BA, CA, CCA, FA, LA, PA, TFA, and 2ABA caused almost negligible changes in emission spectra (Figure S34). The fluorescence intensity changes of $\mathbf{P 2}$ and Ani:BF were also examined upon addition of 5 equiv. of electron deficient organic substances. Figure S35 clearly demonstrates that these electron deficient organic substances did not influence the emission spectra. These results confirm that the good selectivity of sensor $\mathbf{P 2}$ for aniline in the presence of organic acids and electron deficient organic substances. 
Figure S34 Selectivity of $\mathrm{P} 2+\mathrm{Ani}_{\mathrm{B}} \mathrm{BF}_{3}$ mixture $\left(2.0 \times 10^{-5} \mathrm{M}\right)$ towards various organic acids $\left(1 \mathrm{X} 10^{-3} \mathrm{M}\right.$, left). The fluorescence intensity at $540 \mathrm{~nm}$ of sensor $\mathrm{P} 2+\mathrm{Ani}: \mathrm{BF}_{3}$ in the presence of various organic acids (right). 1: 3MPA-3-Mercaptopropanoic acid 2: AA-Acetic acid 3: BABenzoic acid 4: CA-Citric acid 5: CCA-Chloroacetic acid 6: FA-Formic acid 7: LA-Lactic acid 8: PA-Propionic acid 9: TFA-Trifluoroacetic acid and 10: 2ABA-2-Aminobenzoic acid
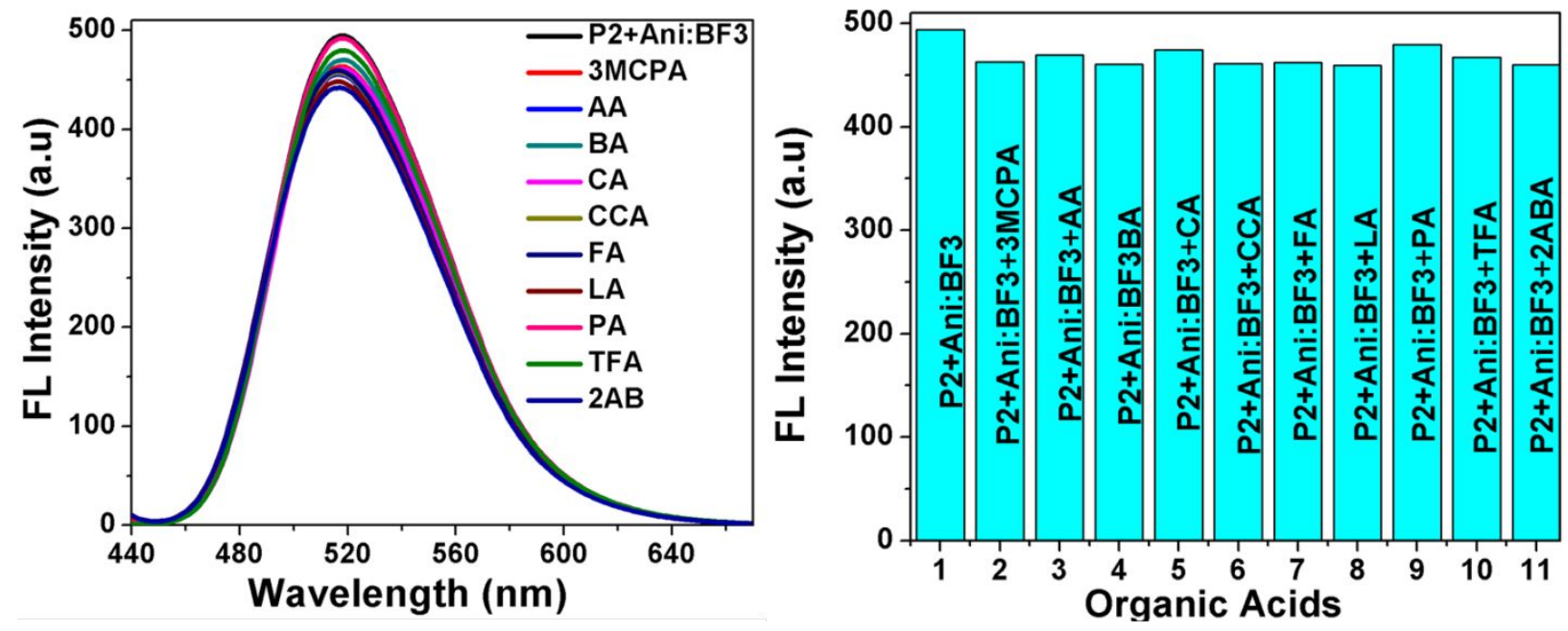

Figure S35 Selectivity of $\mathbf{P 2}\left(2.0 \times 10^{-5} \mathrm{M}\right)$ in the presence of various electron deficient organic substances $\left(1 \times 10^{-3} \mathrm{M}\right.$, left). NB- Nitrobenzene MN- Malanonitrile BN- Benzonitrile NBNitrobenzene ACN- Acetonitrile ACRN- Acrylonitrile PACN-Phenylacetylene
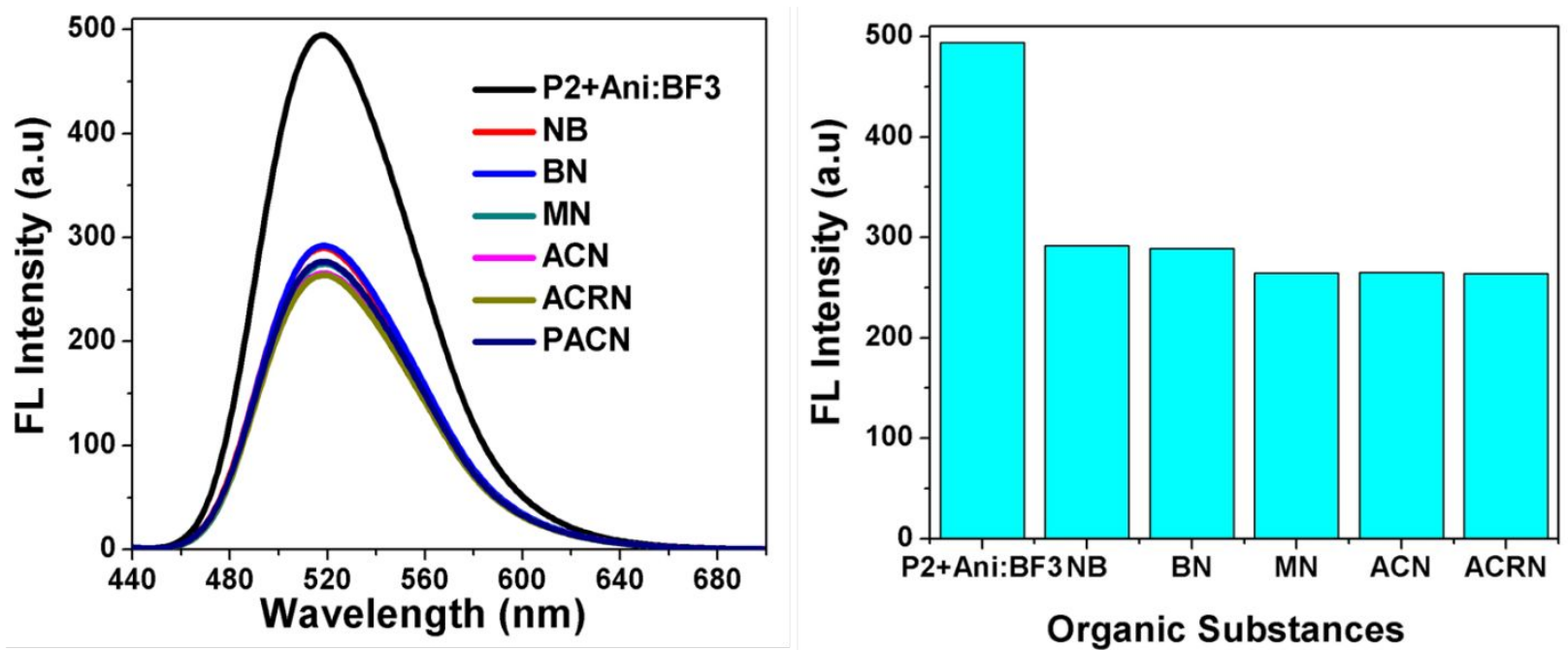
Figure S36. Changes in (a) absorbance and (b) emission intensities of P2 (20 $\mu \mathrm{M})$ after adding $\mathrm{Zn}^{2+}\left(1 \times 10^{-3} \mathrm{M}\right)$ and the excitation wavelength is $405 \mathrm{~nm}$.

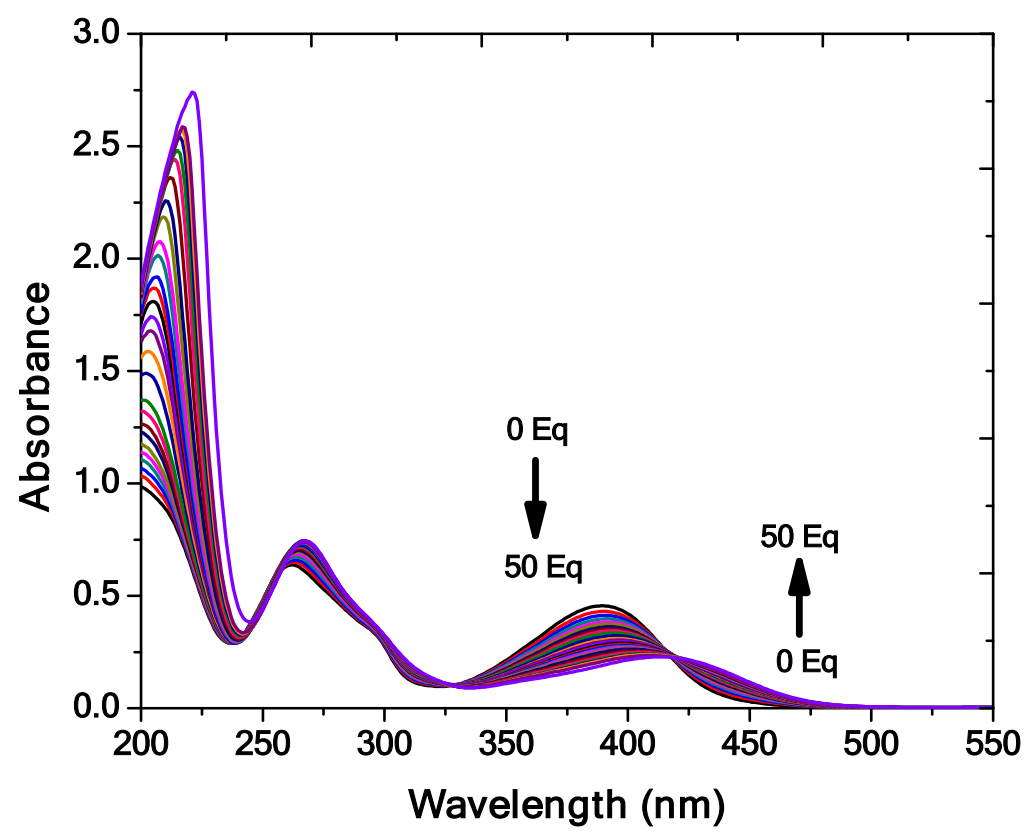

(a)

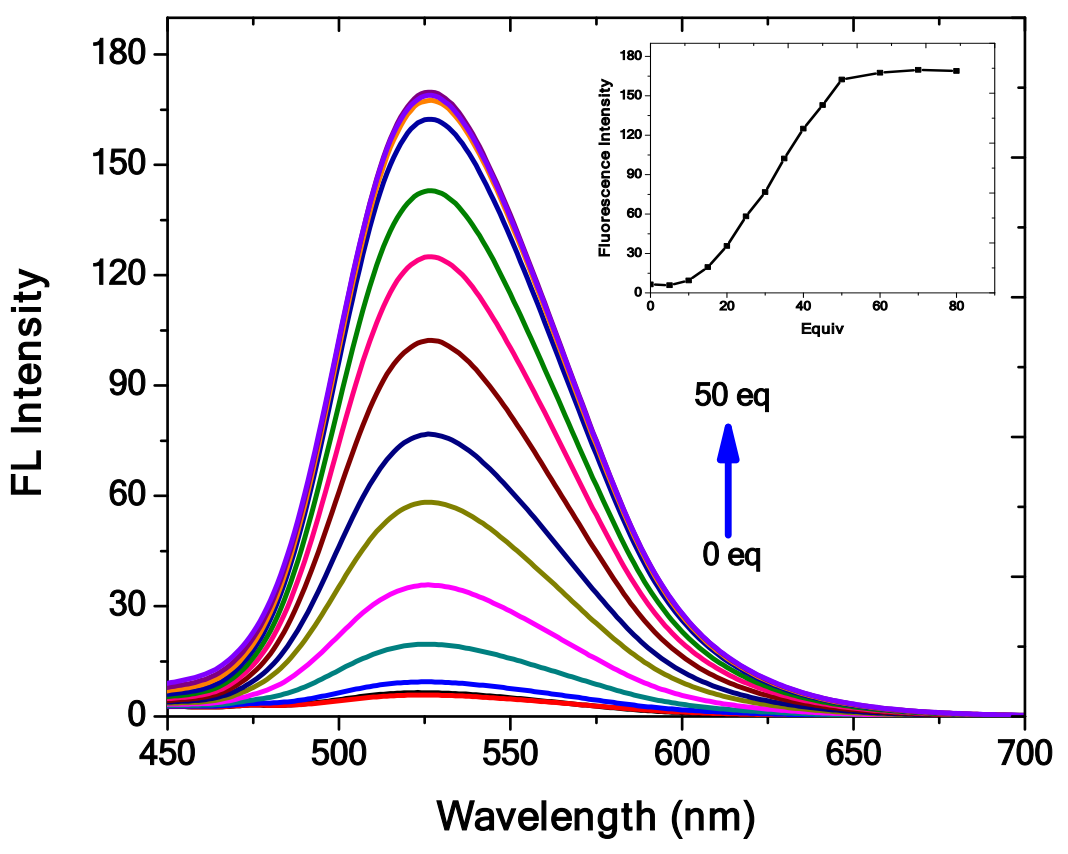

(b) 
Figure S37. Calibration plot of Aniline concentration versus Fluorescence intensity

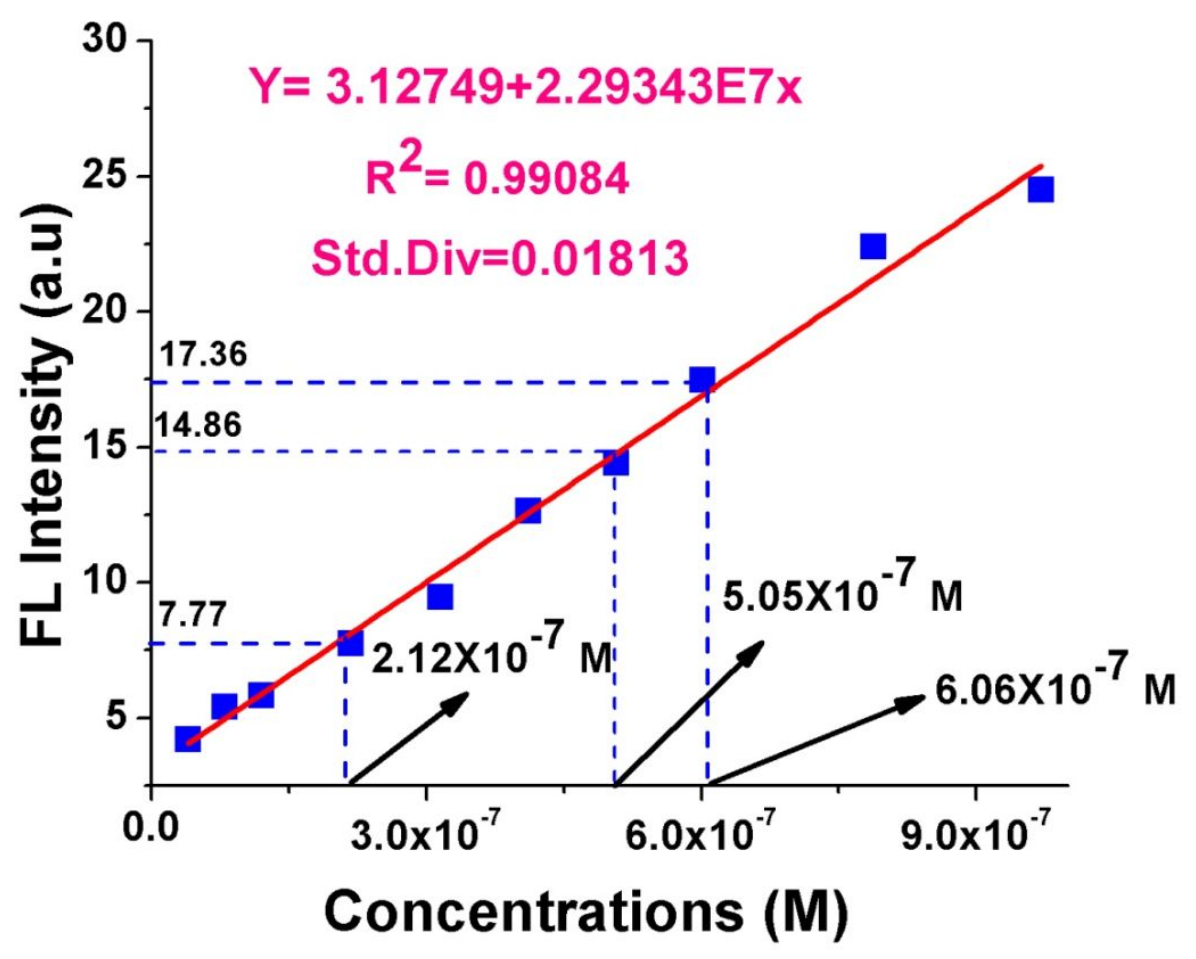

Figure S38. Screening of various metal ions using absorption studies.

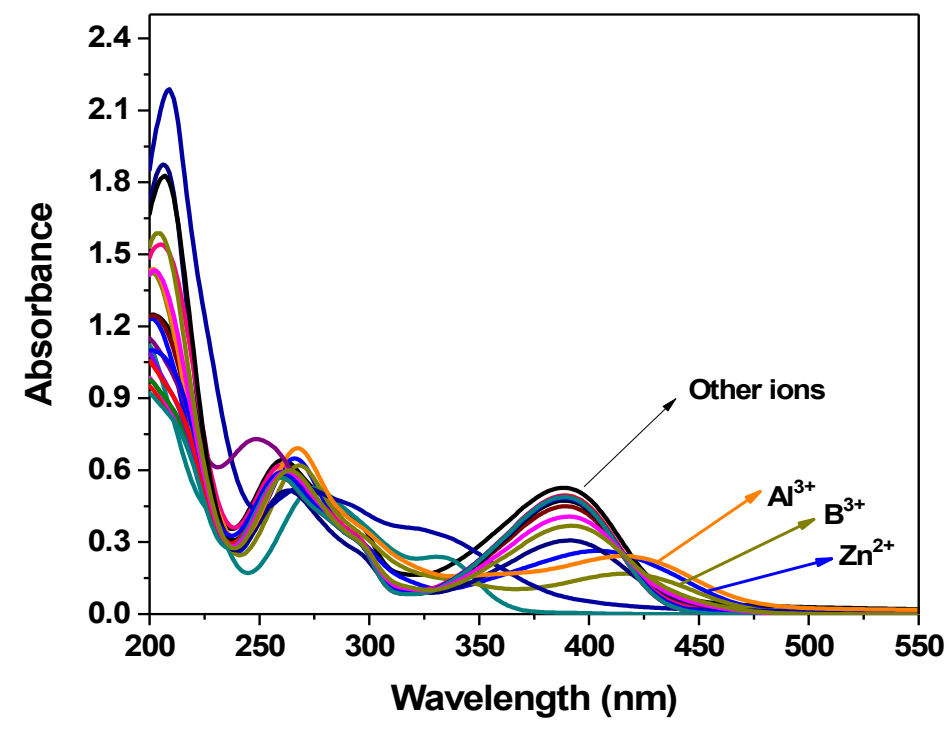




\section{${ }^{1} \mathrm{H}-\mathrm{NMR}$ spectrum of $\mathrm{P} 1$}
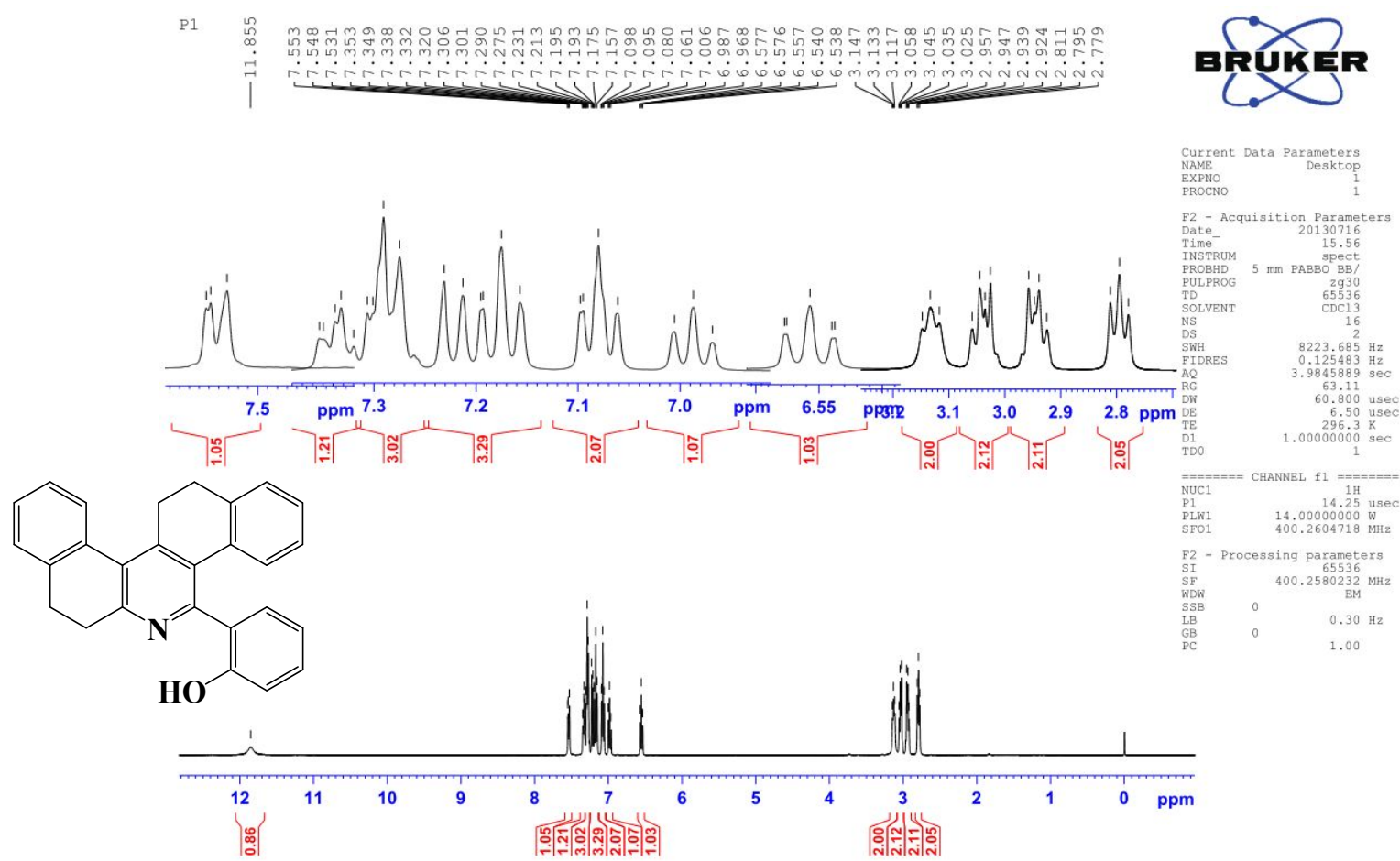

\section{${ }^{13} \mathrm{C}-\mathrm{NMR}$ spectrum of $\mathrm{P} 1$}

P1<smiles>Oc1ccccc1-c1nc2c(c3c1-c1ccccc1CC3)-c1ccccc1CC2</smiles>

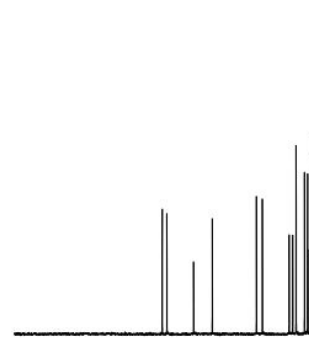

$\begin{array}{llllllllll}180 & 170 & 160 & 150 & 140 & 130 & 120 & 110 & 100 & 90\end{array}$
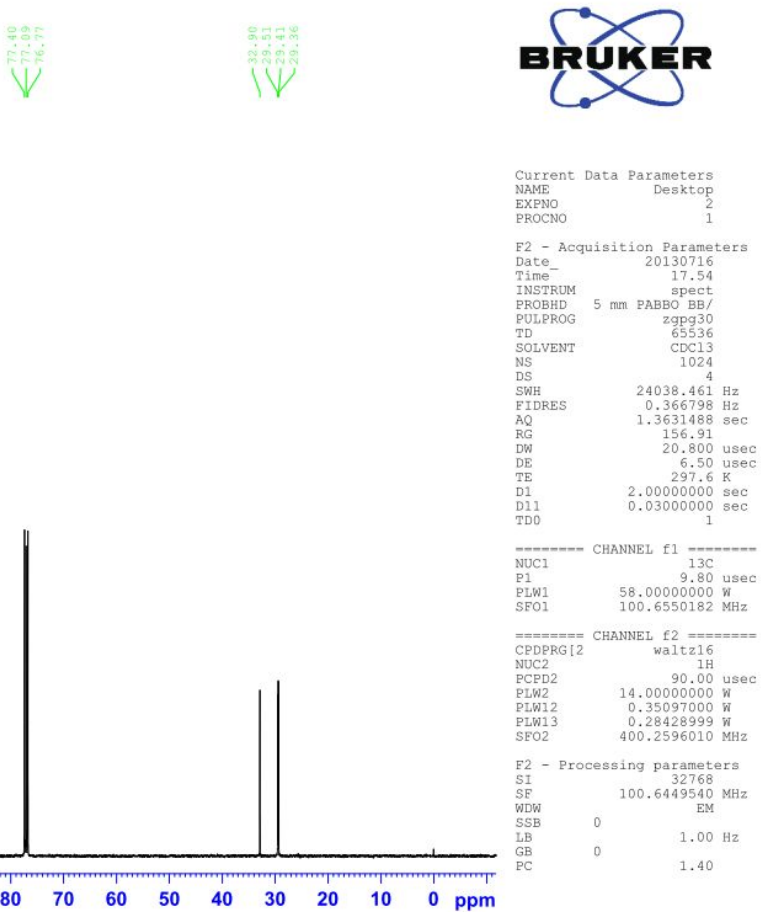
COSY spectrum of P1
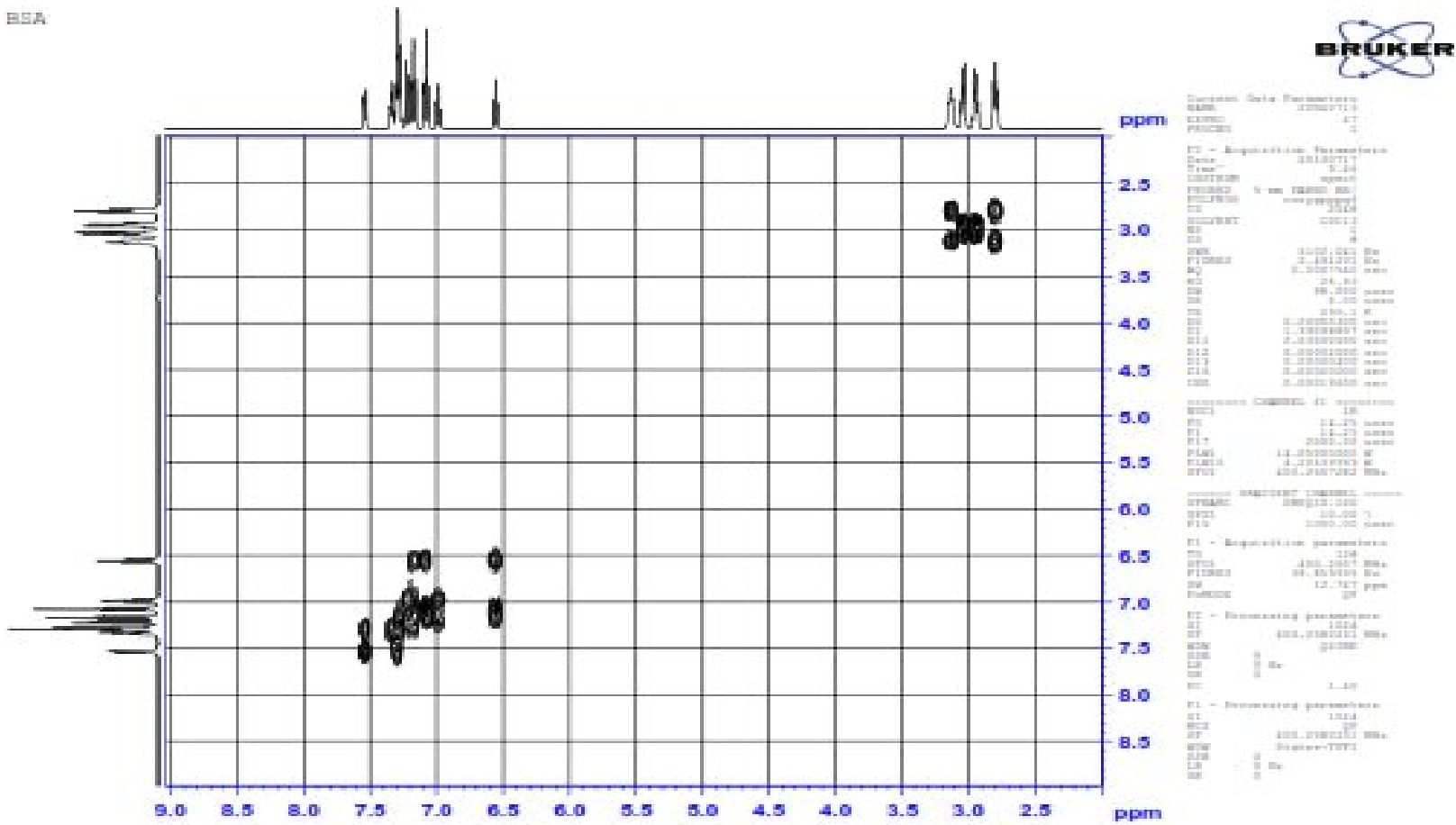

\section{HMQC spectrum of P1}

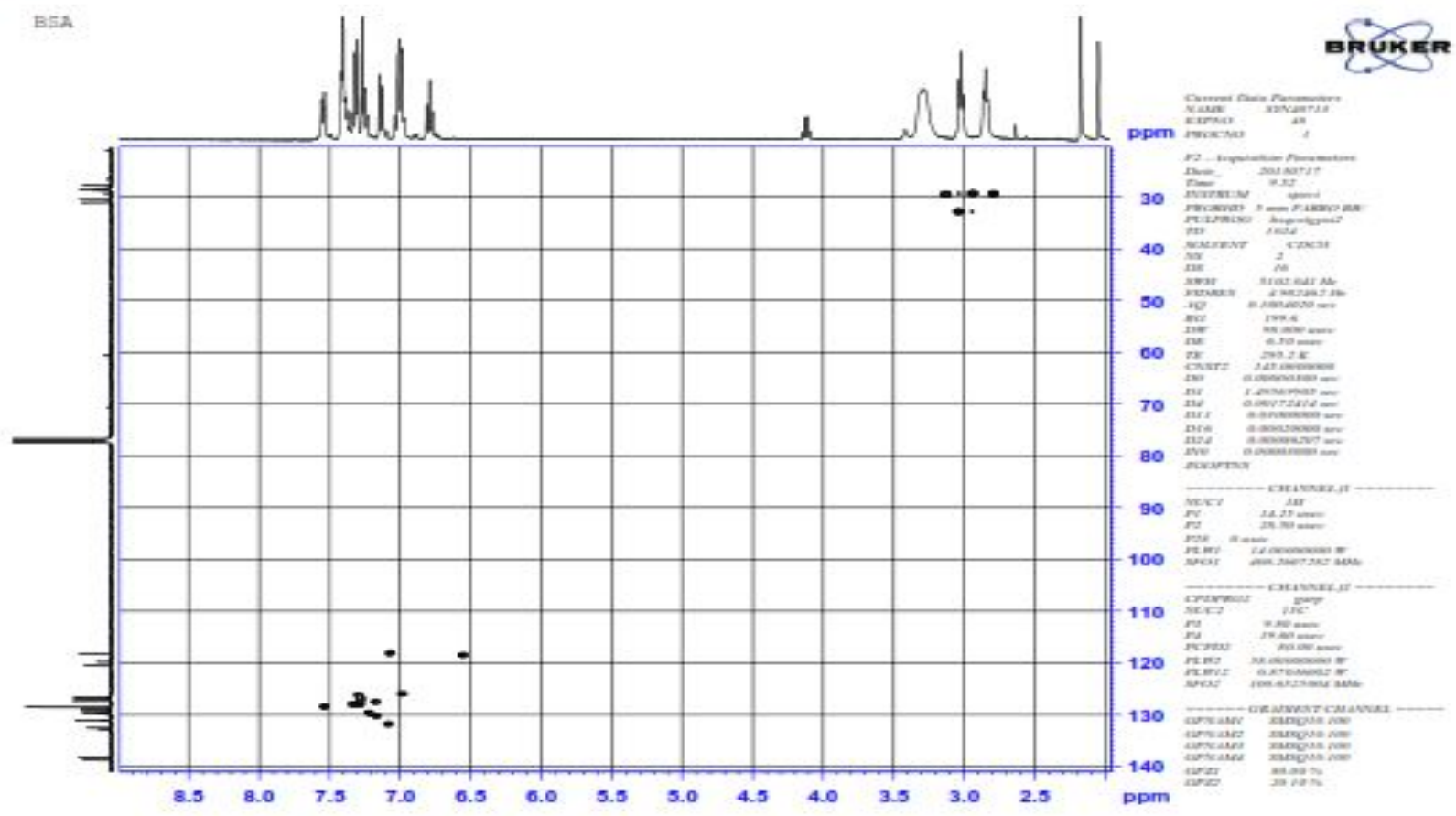




\section{${ }^{1} \mathrm{H}-\mathrm{NMR}$ spectrum of $\mathrm{P2}$}

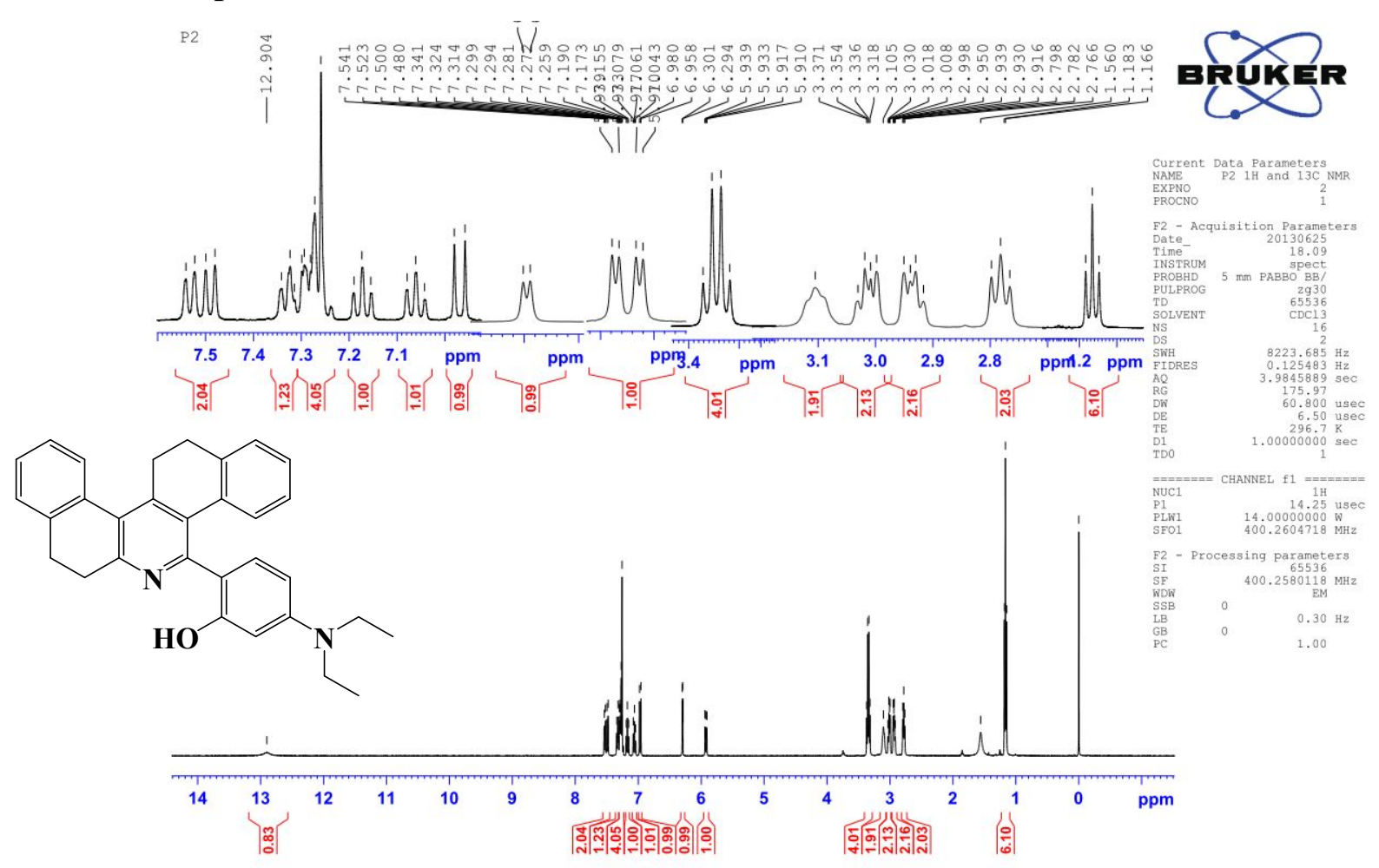

\section{${ }^{13} \mathrm{C}-\mathrm{NMR}$ spectrum of $\mathrm{P} 2$}

2T4DESA

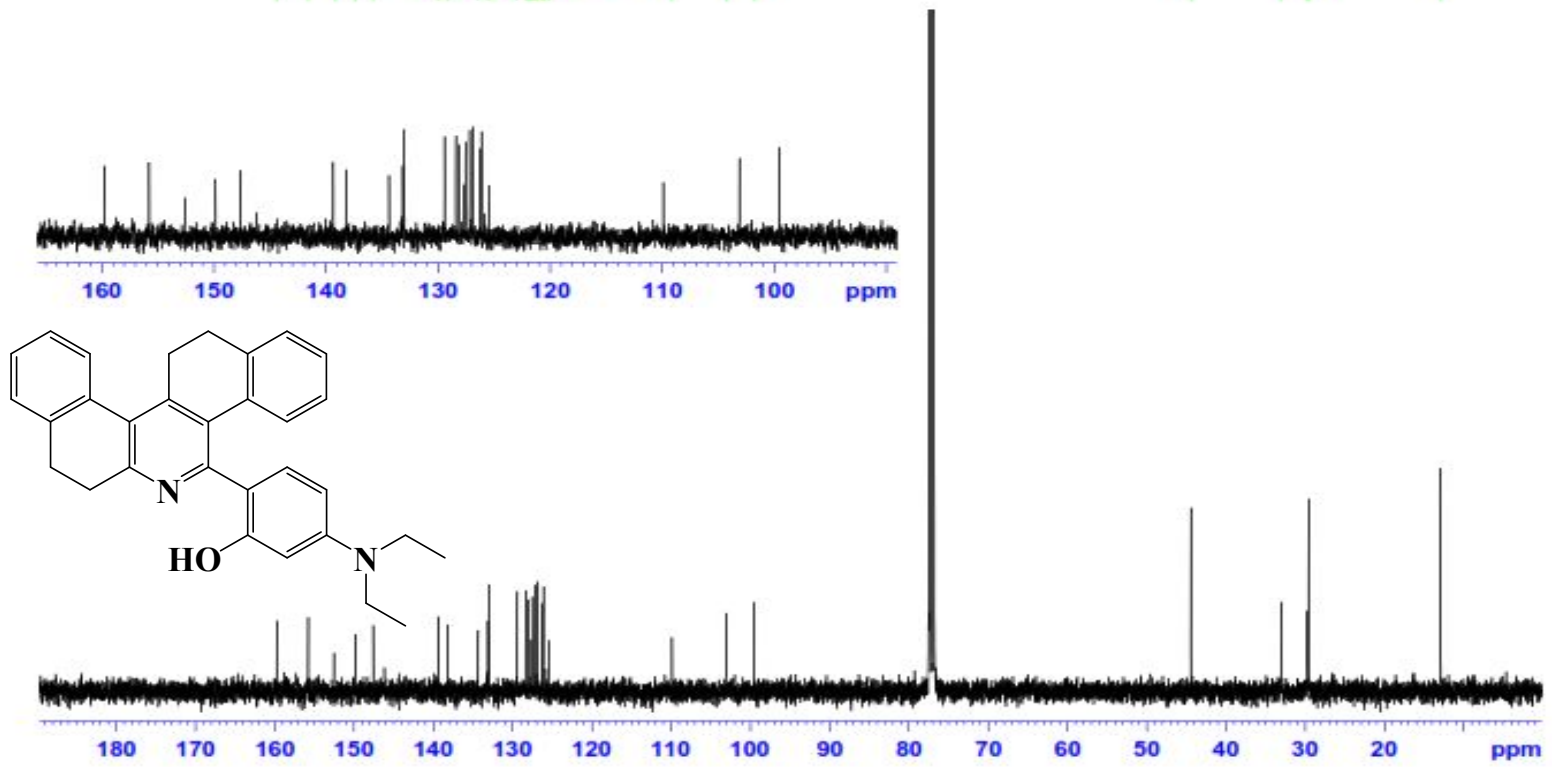




\section{${ }^{1} \mathrm{H}-\mathrm{NMR}$ spectrum of PBC1}

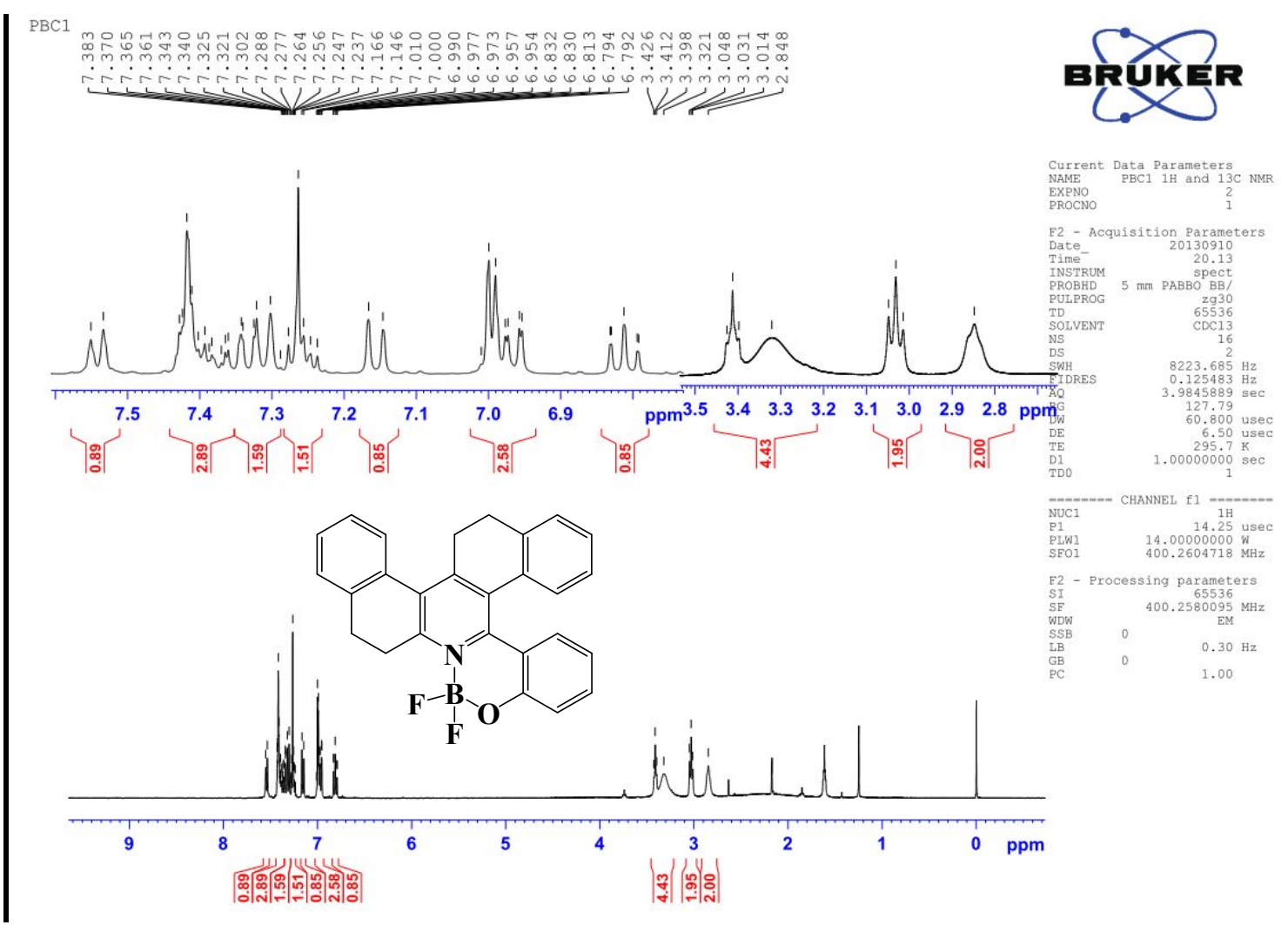

${ }^{13} \mathrm{C}-\mathrm{NMR}$ spectrum of PBC1

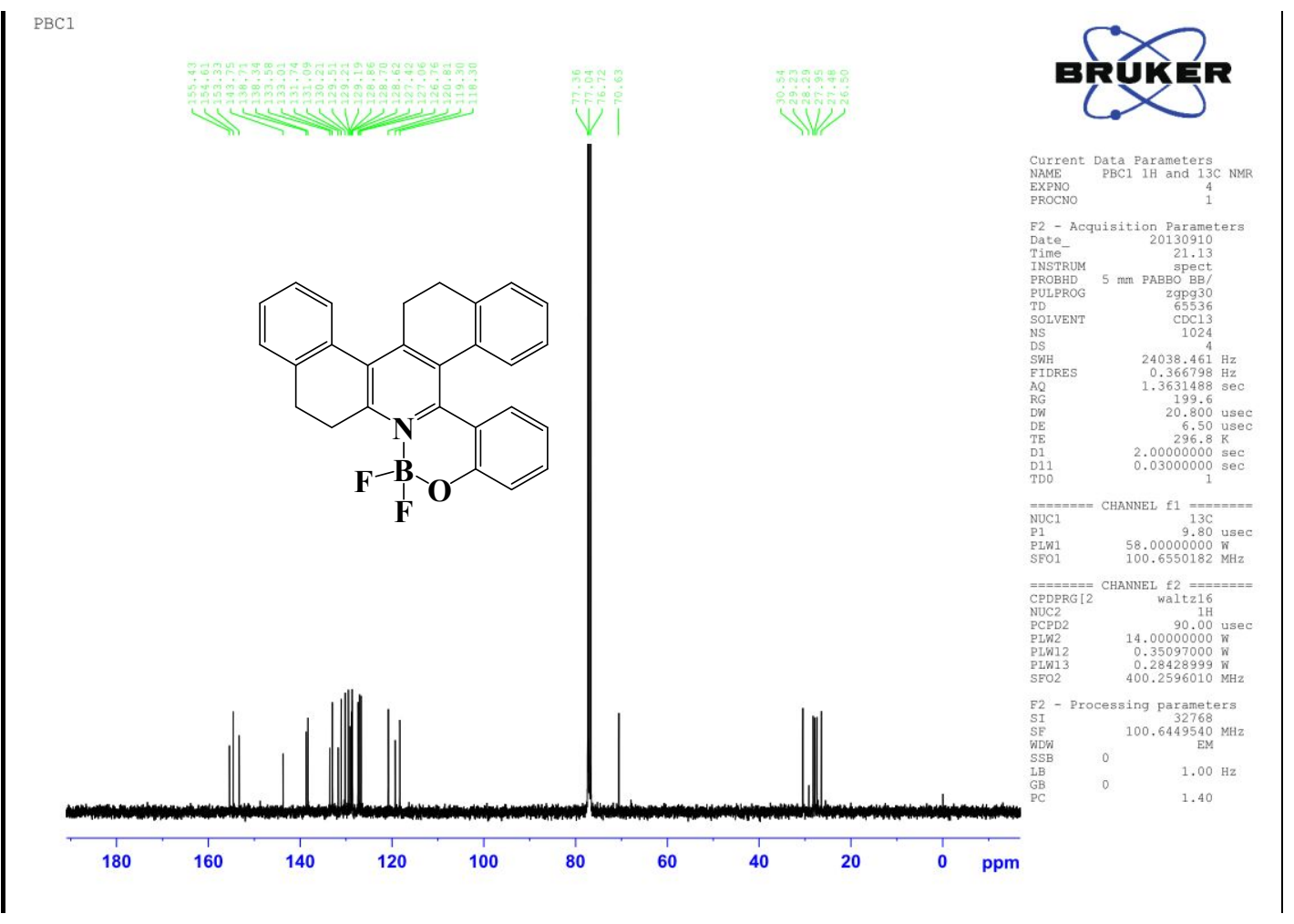


${ }^{11} \mathrm{~B}-\mathrm{NMR}$ spectrum of PBC1

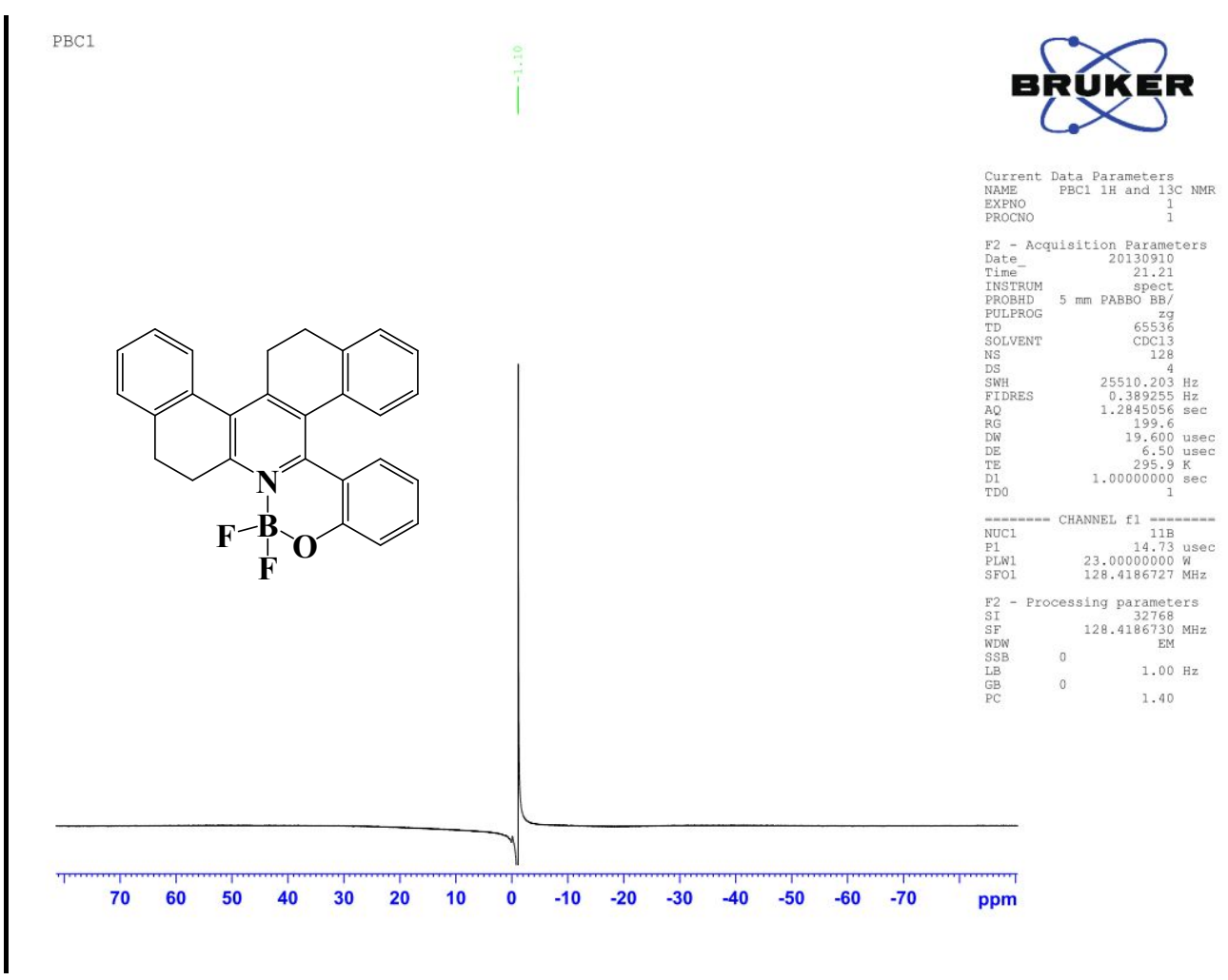

${ }^{19}$ F-NMR spectrum of PBC1

PBC1<smiles>FC1(F)Oc2ccccc2-c2c3c(c4c(c21)-c1ccccc1CC4)-c1ccccc1CC3</smiles>

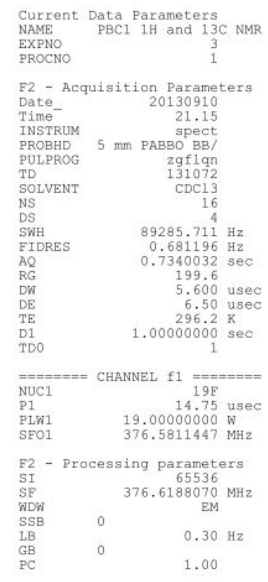




\section{HMQC spectrum of PBC1}

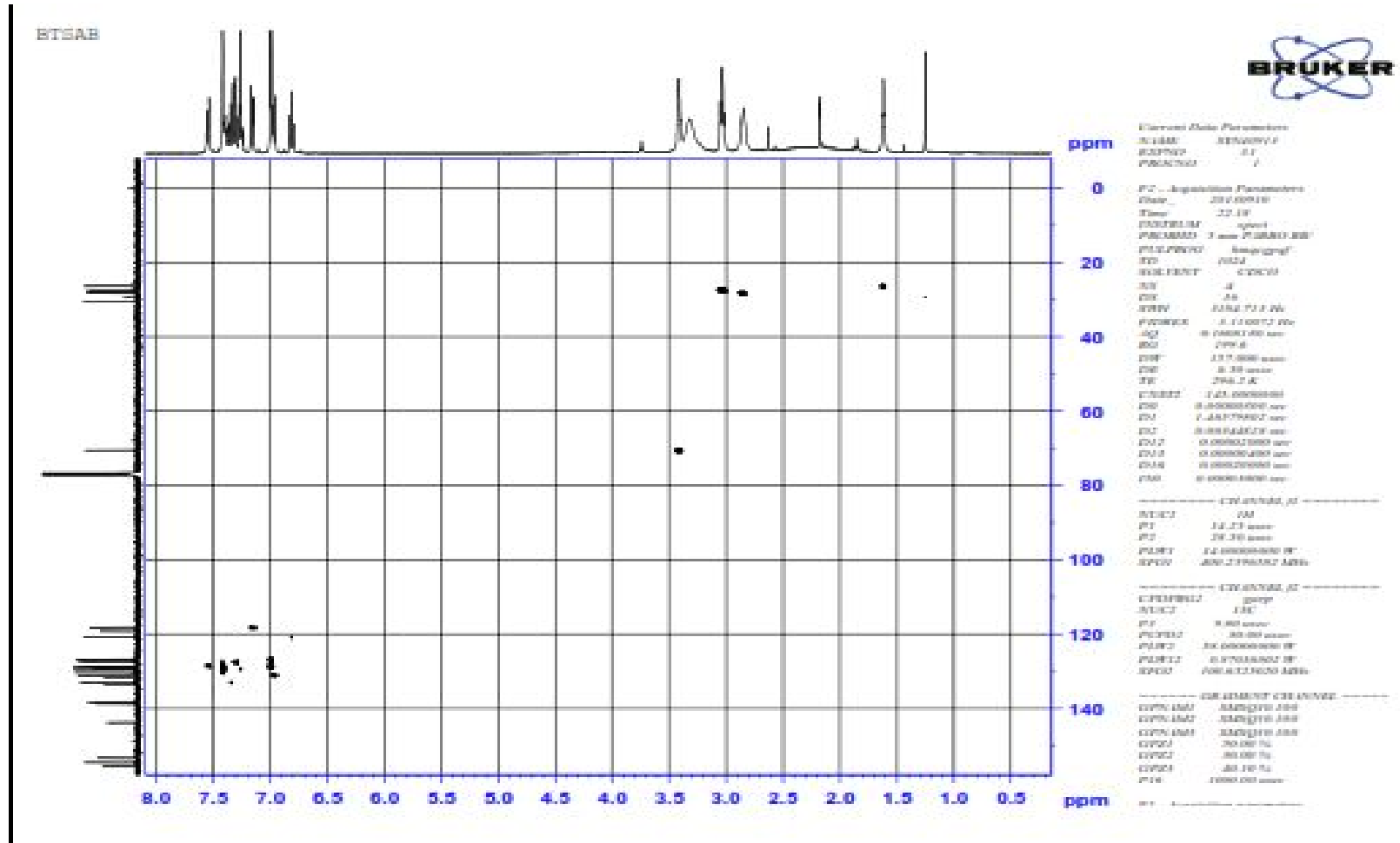

COSY spectrum of PBC1

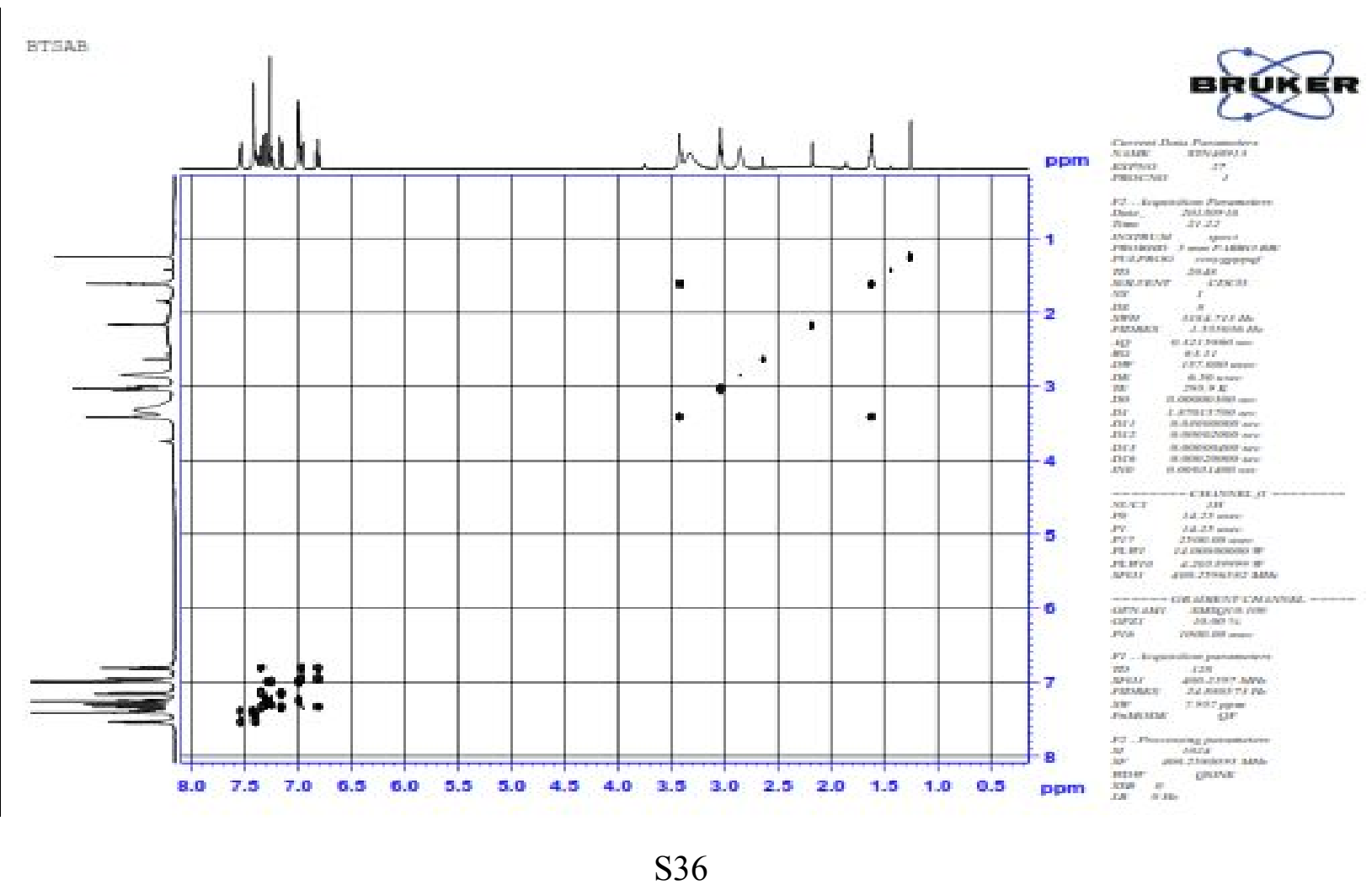




\section{${ }^{1} \mathrm{H}-\mathrm{NMR}$ spectrum of $\mathrm{PBC} 2$}

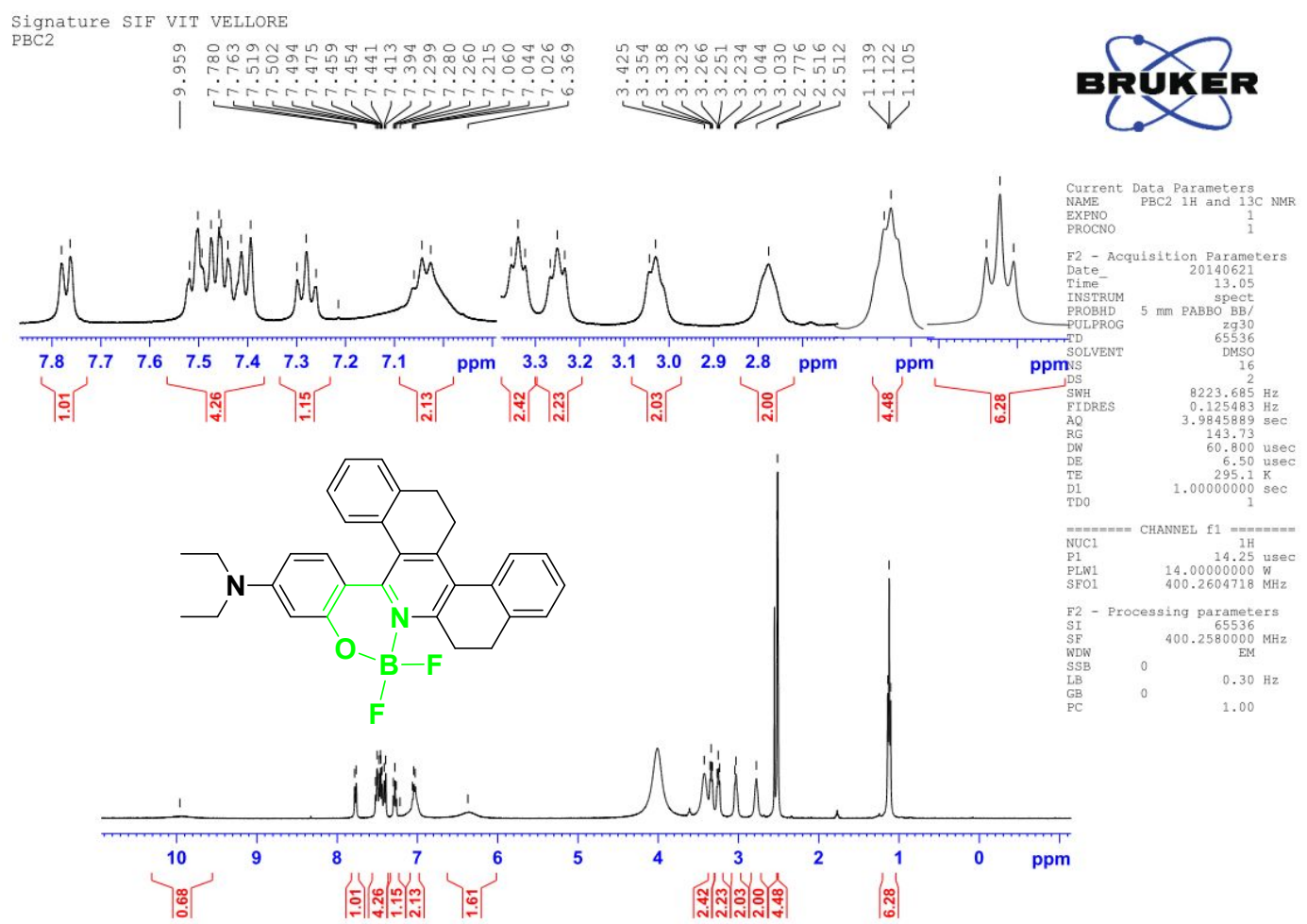

\section{${ }^{19}$ F-NMR spectrum of PBC2}

Signature SIF VIT VELLORE
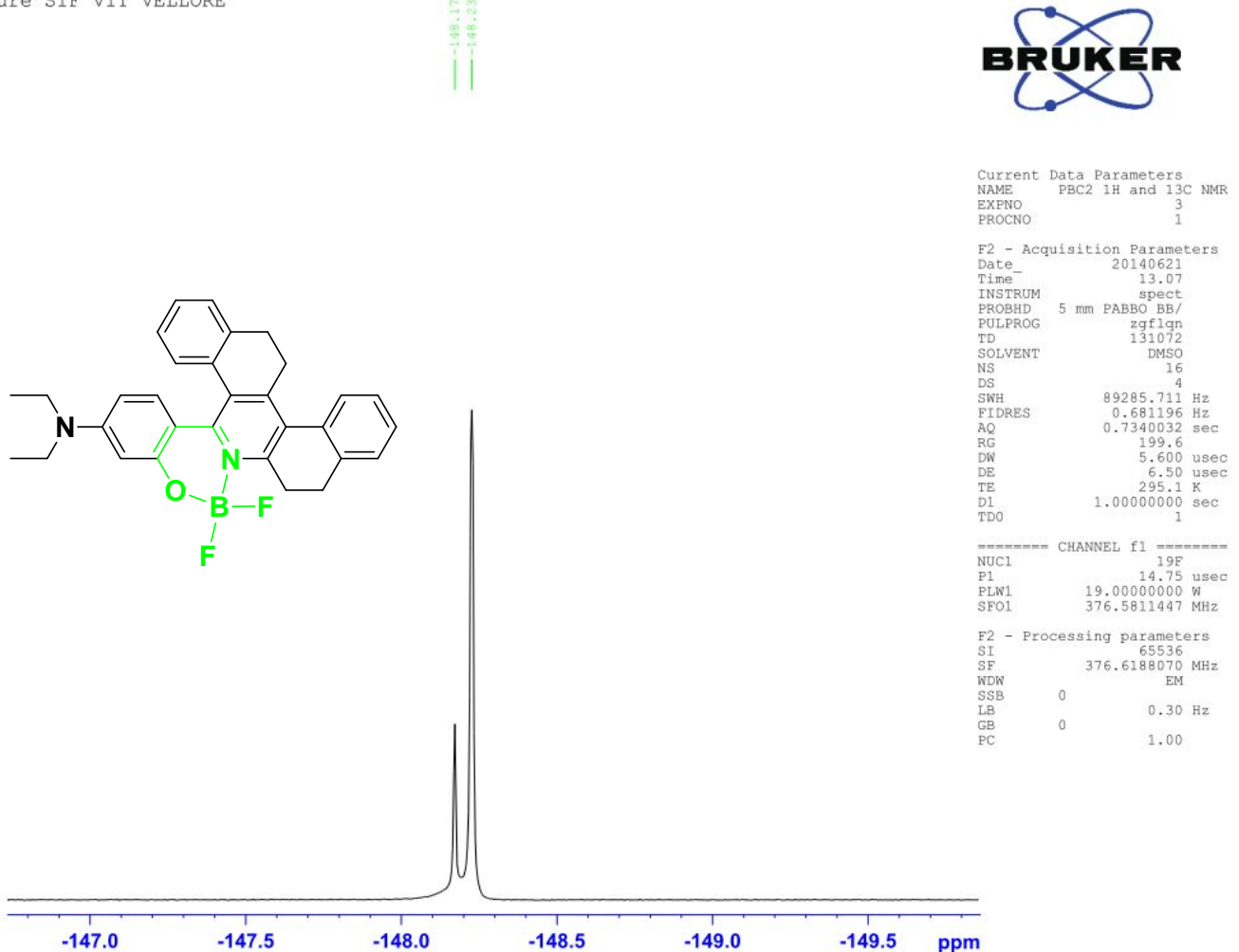


\section{${ }^{1} \mathrm{H}-\mathrm{NMR}$ spectrum of $\mathrm{P} 2 \mathrm{ZnCl} 2$}

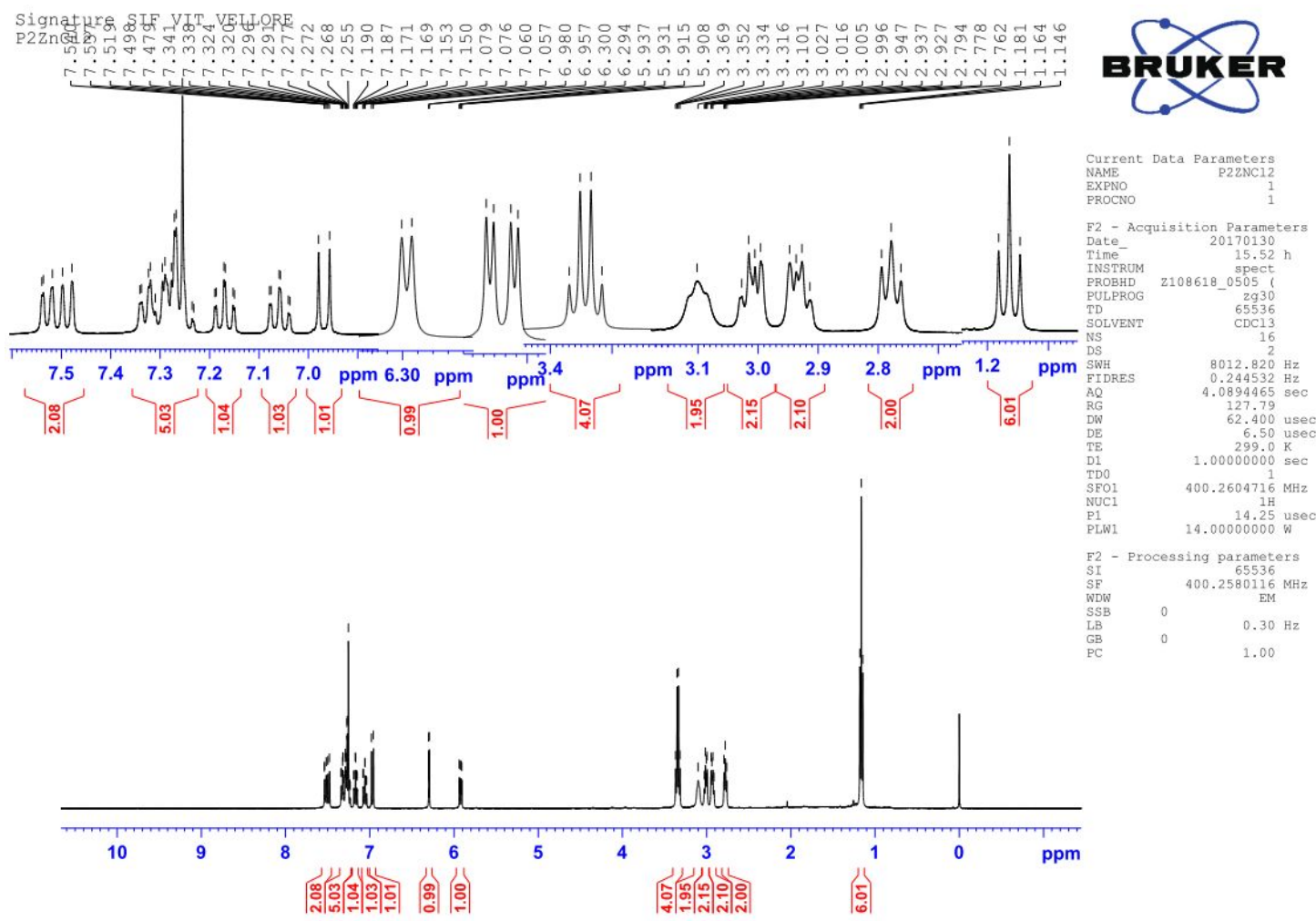

${ }^{13} \mathrm{C}$-NMR spectrum of $\mathrm{P} 2 \mathrm{ZnCl} 2$

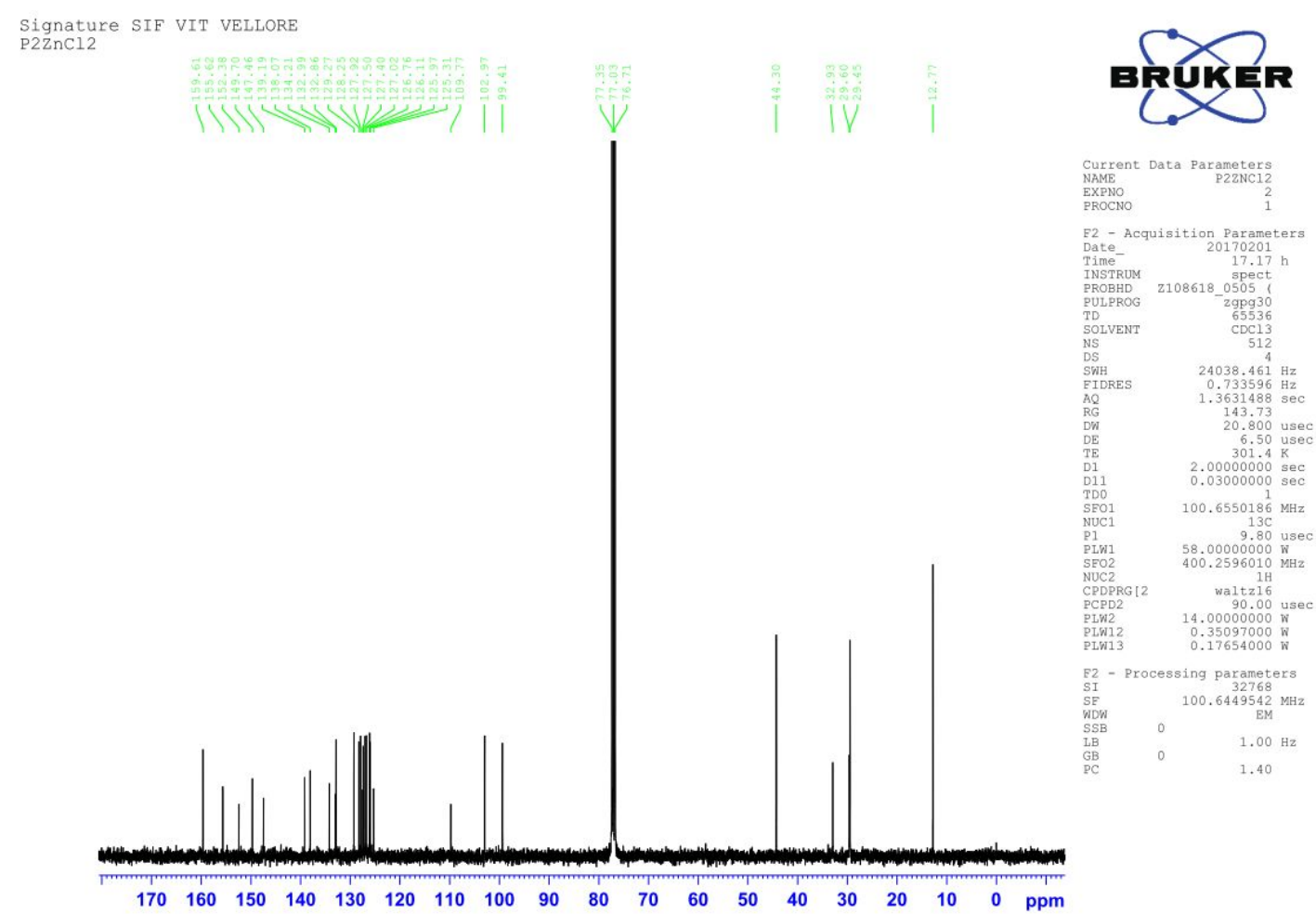


$\underline{\text { SI Table S1 The comparisons of aniline detection sensor of } \mathbf{P 2} \text { in the previous and present works }}$

\begin{tabular}{|c|c|c|c|c|c|}
\hline $\begin{array}{c}\text { Molecular structure of the } \\
\text { sensor }\end{array}$ & $\begin{array}{c}\text { Solvent/supported } \\
\text { systems }\end{array}$ & Method used & $\begin{array}{l}\text { Detection } \\
\text { limit }\end{array}$ & Application & References \\
\hline 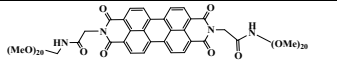 & Detection of amine vapor & "Fluorescence-off" & $24 \mathrm{nM}$ & $\begin{array}{c}\text { Vapor } \\
\text { detection }\end{array}$ & $\begin{array}{l}\text { J. Org. Chem. 2011, 76, } \\
\text { 6101-6107 }\end{array}$ \\
\hline & Acetonitrile & Colorimetric & $1.2 \mu \mathrm{M}$ & $\begin{array}{r}\text { AND logic } \\
\text { gate and Test } \\
\text { Paper strips }\end{array}$ & $\begin{array}{l}\text { Journal of Photochemistry } \\
\text { and Photobiology A: } \\
\text { Chemistry. 2012, 238 1-6 }\end{array}$ \\
\hline & THF & "Fluorescence-off" & $3.06 \mu \mathrm{M}$ & $\begin{array}{c}\text { Vapor } \\
\text { detection and } \\
\text { Test Paper } \\
\text { strips } \\
\end{array}$ & $\begin{array}{c}\text { Chem. Commun., 2013, 49, } \\
\text { 11266-11268 }\end{array}$ \\
\hline & Detection of amine gas & "Fluorescence-on" & $3 \mathrm{mM}$ & $\begin{array}{l}\text { Filter paper } \\
\text { strip }\end{array}$ & $\begin{array}{c}\text { Chem. Commun., 2013, 49, } \\
4848-4850\end{array}$ \\
\hline & Acetonitrile & "Fluorescence-on" & $25 \mu \mathrm{M}$ & $\begin{array}{l}\text { Biomolecule } \\
\text { labeling }\end{array}$ & $\begin{array}{c}\text { J. Am. Chem. Soc. 2014, 136, } \\
15493\end{array}$ \\
\hline & Water/THF (9:1) & "Fluorescence-on" & $3.76 \mu \mathrm{M}$ & $\begin{array}{l}\text { Filter paper } \\
\text { strip }\end{array}$ & $\begin{array}{l}\text { J. Org. Chem. 2015, 80, } \\
5159-5166\end{array}$ \\
\hline & Detection of amine vapor & "Fluorescence-on" & $29 \mu \mathrm{M}$ & $\begin{array}{l}\text { Ammonia } \\
\text { vapor and } \\
\text { Food spoiling } \\
\text { sensor using } \\
\text { saury fish }\end{array}$ & ACS Sens, 2016, 1, 179-184 \\
\hline Spiropyran derivatives & THF & "Fluorescence-off" & $0.1 \mu \mathrm{M}$ & $\begin{array}{c}\text { Cellulose test } \\
\text { Paper }\end{array}$ & $\begin{array}{c}\text { RSC Adv., 2016, 6, 83312- } \\
83320 \\
\end{array}$ \\
\hline & DMF & "Fluorescence-off" & $0.09 \mathrm{mM}$ & & ACS Sens, 2017, 2, 842-847 \\
\hline & THF & "Fluorescence-on" & $12.65 \mathrm{nM}$ & $\begin{array}{c}\text { Aluminum- } \\
\text { backed TLC } \\
\text { plate and } \\
\text { Whatman } \\
\text { filter paper } \\
\text { strips in } \\
\text { solutions and } \\
\text { vapor phase }\end{array}$ & Present work \\
\hline
\end{tabular}


SI Table S2. Crystal data and structure refinement for P1_cif

\begin{tabular}{|c|c|c|}
\hline Identification code & $\mathrm{P} 1$ & \\
\hline Empirical formula & $\mathrm{C}_{27} \mathrm{H}_{21} \mathrm{~N} \mathrm{O}$ & \\
\hline Formula weight & 375.45 & \\
\hline Temperature & $293(2) \mathrm{K}$ & \\
\hline Wavelength & $0.71073 \AA$ & \\
\hline Crystal system & MONOCLINIC & \\
\hline Space group & $\mathrm{P} 2{ }_{1} / \mathrm{a}$ & \\
\hline \multirow[t]{3}{*}{ Unit cell dimensions } & $\mathrm{a}=10.060(5) \AA$ & $\alpha=90.000(5)^{\circ}$ \\
\hline & $\mathrm{b}=12.876(5) \AA$ & $\beta=101.971(5)^{\circ}$. \\
\hline & $\mathrm{c}=15.353(5) \AA$ & $\gamma=90.000(5)^{\circ}$ \\
\hline Volume & $1945.5(14) \AA^{3}$ & \\
\hline $\mathrm{Z}$ & 4 & \\
\hline Density (calculated) & $1.282 \mathrm{Mg} / \mathrm{m}^{3}$ & \\
\hline Absorption coefficient & $0.077 \mathrm{~mm}^{-1}$ & \\
\hline $\mathrm{F}(000)$ & 792 & \\
\hline Theta range for data collection & 2.08 to $30.61^{\circ}$. & \\
\hline Index ranges & $\begin{array}{l}-12<=\mathrm{h}<=14,-18<=\mathrm{k}<=14,- \\
19<=1<=21\end{array}$ & \\
\hline Reflections collected & 23424 & \\
\hline Independent reflections & $5964[\mathrm{R}($ int $)=0.0365]$ & \\
\hline Completeness to theta $=30.61^{\circ}$ & $99.4 \%$ & \\
\hline Refinement method & Full-matrix least-squares on $\mathrm{F}^{2}$ & \\
\hline Data / restraints / parameters & $5964 / 0 / 262$ & \\
\hline Goodness-of-fit on $\mathrm{F}^{2}$ & 0.961 & \\
\hline Final R indices [I>2sigma(I)] & $\mathrm{R} 1=0.0511, \mathrm{wR} 2=0.1314$ & \\
\hline $\mathrm{R}$ indices (all data) & $\mathrm{R} 1=0.0944, \mathrm{wR} 2=0.1608$ & \\
\hline Largest diff. peak and hole & 0.248 and -0.221 e. $\AA^{-3}$ & \\
\hline
\end{tabular}

Atomic coordinates $\left(x 1^{4}\right)$ and equivalent isotropic displacement parameters $\left(\AA^{2} \times 10^{3}\right)$ for P1_cif. $U(e q)$ is defined as one third of the trace of the orthogonalized $U i j$ tensor

\begin{tabular}{|l|l|l|l|l|}
\hline Atom & $\mathrm{x}$ & $\mathrm{y}$ & $\mathrm{z}$ & $\mathrm{U}(\mathrm{eq})$ \\
\hline $\mathrm{C}(1)$ & $9597(1)$ & $3547(1)$ & $828(1)$ & $34(1)$ \\
\hline $\mathrm{C}(2)$ & $8473(2)$ & $2780(1)$ & $549(1)$ & $45(1)$ \\
\hline $\mathrm{C}(3)$ & $9099(2)$ & $1726(1)$ & $430(1)$ & $30(1)$ \\
\hline $\mathrm{C}(4)$ & $9992(2)$ & $1816(1)$ & $-238(1)$ & $45(1)$ \\
\hline $\mathrm{C}(5)$ & $10053(2)$ & $1032(1)$ & $-845(1)$ & $49(1)$ \\
\hline $\mathrm{C}(6)$ & $10822(2)$ & $1131(1)$ & $-1485(1)$ & $48(1)$ \\
\hline $\mathrm{C}(7)$ & $11483(2)$ & $2053(1)$ & $-1557(1)$ & $40(1)$ \\
\hline $\mathrm{C}(8)$ & $11436(2)$ & $2849(1)$ & $-959(1)$ & $34(1)$ \\
\hline $\mathrm{C}(9)$ & $10747(1)$ & $2733(1)$ & $-264(1)$ & $32(1)$ \\
\hline $\mathrm{C}(10)$ & $10734(1)$ & $3527(1)$ & $433(1)$ & $31(1)$ \\
\hline $\mathrm{C}(11)$ & $11782(1)$ & $4229(1)$ & $757(1)$ & $39(1)$ \\
\hline $\mathrm{C}(12)$ & $13119(1)$ & $4218(1)$ & $457(1)$ & $46(1)$ \\
\hline $\mathrm{C}(13)$ & $14275(2)$ & $4561(1)$ & $1203(1)$ & \\
\hline $\mathrm{C}(14)$ & $13973(1)$ & $5623(1)$ & $1507(1)$ & $40(1)$ \\
\hline $\mathrm{C}(15)$ & $14906(2)$ & $6428(1)$ & $1629(1)$ & $53(1)$ \\
\hline
\end{tabular}




\begin{tabular}{|l|l|l|l|l|}
\hline $\mathrm{C}(16)$ & $14543(2)$ & $7409(2)$ & $1850(1)$ & $58(1)$ \\
\hline $\mathrm{C}(17)$ & $13233(2)$ & $7597(1)$ & $1956(1)$ & $50(1)$ \\
\hline $\mathrm{C}(18)$ & $12289(2)$ & $6805(1)$ & $1838(1)$ & $38(1)$ \\
\hline $\mathrm{C}(19)$ & $12650(1)$ & $5806(1)$ & $1622(1)$ & $34(1)$ \\
\hline $\mathrm{C}(20)$ & $11639(1)$ & $4951(1)$ & $1425(1)$ & $31(1)$ \\
\hline $\mathrm{C}(21)$ & $10525(1)$ & $4841(1)$ & $1836(1)$ & $35(1)$ \\
\hline $\mathrm{C}(22)$ & $10369(2)$ & $5329(1)$ & $2685(1)$ & $46(1)$ \\
\hline $\mathrm{C}(23)$ & $11486(2)$ & $5590(1)$ & $3349(1)$ & $65(1)$ \\
\hline $\mathrm{C}(24)$ & $11349(2)$ & $6012(2)$ & $4150(1)$ & $74(1)$ \\
\hline $\mathrm{C}(25)$ & $10064(2)$ & $6183(2)$ & $4305(1)$ & $66(1)$ \\
\hline $\mathrm{C}(26)$ & $8950(2)$ & $5902(2)$ & $3683(1)$ & $43(1)$ \\
\hline $\mathrm{C}(27)$ & $9078(2)$ & $5453(1)$ & $2885(1)$ & $54(1)$ \\
\hline $\mathrm{O}(2)$ & $7919(1)$ & $5146(1)$ & $2322(1)$ & $34(1)$ \\
\hline $\mathrm{N}(1)$ & $9510(1)$ & $4172(1)$ & $1502(1)$ & \\
\hline
\end{tabular}

Anisotropic displacement parameters $\left(\AA^{2} \times 10^{3}\right)$ for P1_cif. The anisotropic displacement factor exponent takes the form: $-2 \square^{2}\left[h^{2} a^{* 2} U^{11}+\ldots+2 h k^{*} b^{*} U^{12}\right]$

\begin{tabular}{|c|c|c|c|c|c|c|}
\hline Atom & $\mathrm{U}^{11}$ & $\mathrm{U}^{22}$ & $\mathrm{U}^{33}$ & $\mathrm{U}^{23}$ & $\mathrm{U}^{13}$ & $\mathrm{U}^{12}$ \\
\hline $\mathrm{C}(1)$ & $36(1)$ & $33(1)$ & $34(1)$ & $2(1)$ & 11(1) & $-1(1)$ \\
\hline $\mathrm{C}(2)$ & $42(1)$ & $48(1)$ & $49(1)$ & $-7(1)$ & $20(1)$ & $-13(1)$ \\
\hline $\mathrm{C}(3)$ & $65(1)$ & $39(1)$ & $52(1)$ & $-6(1)$ & $26(1)$ & $-16(1)$ \\
\hline $\mathrm{C}(4)$ & $45(1)$ & $35(1)$ & $36(1)$ & $-2(1)$ & $10(1)$ & $-2(1)$ \\
\hline $\mathrm{C}(5)$ & $53(1)$ & $37(1)$ & $44(1)$ & $-6(1)$ & $7(1)$ & $-2(1)$ \\
\hline $\mathrm{C}(6)$ & $57(1)$ & $50(1)$ & $39(1)$ & $-12(1)$ & $7(1)$ & $7(1)$ \\
\hline$C(7)$ & $51(1)$ & $61(1)$ & $34(1)$ & $-6(1)$ & $14(1)$ & $5(1)$ \\
\hline $\mathrm{C}(8)$ & $40(1)$ & $44(1)$ & $36(1)$ & $0(1)$ & $12(1)$ & $0(1)$ \\
\hline $\mathrm{C}(9)$ & $35(1)$ & $34(1)$ & $33(1)$ & $-1(1)$ & $9(1)$ & $2(1)$ \\
\hline $\mathrm{C}(10)$ & $35(1)$ & $30(1)$ & $32(1)$ & $2(1)$ & $12(1)$ & 1(1) \\
\hline $\mathrm{C}(11)$ & $32(1)$ & $31(1)$ & $33(1)$ & $2(1)$ & 11(1) & $2(1)$ \\
\hline $\mathrm{C}(12)$ & $35(1)$ & $40(1)$ & $45(1)$ & $-7(1)$ & $15(1)$ & $-1(1)$ \\
\hline$C(13)$ & $32(1)$ & $51(1)$ & $58(1)$ & $-9(1)$ & $12(1)$ & $2(1)$ \\
\hline $\mathrm{C}(14)$ & $33(1)$ & $46(1)$ & $43(1)$ & $-7(1)$ & $8(1)$ & $-2(1)$ \\
\hline$C(15)$ & $35(1)$ & $64(1)$ & $63(1)$ & $-16(1)$ & $14(1)$ & $-12(1)$ \\
\hline$C(16)$ & $50(1)$ & $55(1)$ & 71(1) & $-17(1)$ & $16(1)$ & $-22(1)$ \\
\hline $\mathrm{C}(17)$ & $53(1)$ & $38(1)$ & $58(1)$ & $-10(1)$ & 11(1) & $-9(1)$ \\
\hline $\mathrm{C}(18)$ & $37(1)$ & $37(1)$ & $42(1)$ & $-4(1)$ & $9(1)$ & $-1(1)$ \\
\hline$C(19)$ & $32(1)$ & $36(1)$ & $34(1)$ & $-2(1)$ & $7(1)$ & $-1(1)$ \\
\hline $\mathrm{C}(20)$ & $30(1)$ & $30(1)$ & $34(1)$ & 1(1) & $8(1)$ & $2(1)$ \\
\hline $\mathrm{C}(21)$ & $33(1)$ & $30(1)$ & $33(1)$ & $2(1)$ & $9(1)$ & $4(1)$ \\
\hline $\mathrm{C}(22)$ & $42(1)$ & $30(1)$ & $35(1)$ & 1(1) & $14(1)$ & $3(1)$ \\
\hline $\mathrm{C}(23)$ & $49(1)$ & $50(1)$ & $40(1)$ & $-5(1)$ & $13(1)$ & $-1(1)$ \\
\hline $\mathrm{C}(24)$ & $79(1)$ & $71(1)$ & $43(1)$ & $-16(1)$ & $14(1)$ & $-11(1)$ \\
\hline $\mathrm{C}(25)$ & $98(2)$ & $78(1)$ & $56(1)$ & $-26(1)$ & $39(1)$ & $-8(1)$ \\
\hline
\end{tabular}




\begin{tabular}{|c|c|c|c|c|c|c|}
\hline $\mathrm{C}(26)$ & $72(1)$ & $68(1)$ & $69(1)$ & $-15(1)$ & $44(1)$ & $3(1)$ \\
\hline $\mathrm{C}(27)$ & $47(1)$ & $40(1)$ & $48(1)$ & $0(1)$ & $23(1)$ & $4(1)$ \\
\hline $\mathrm{O}(2)$ & $40(1)$ & $69(1)$ & $60(1)$ & $-4(1)$ & $23(1)$ & $3(1)$ \\
\hline $\mathrm{N}(1)$ & $35(1)$ & $34(1)$ & $36(1)$ & $0(1)$ & $13(1)$ & $-1(1)$ \\
\hline
\end{tabular}

SI Table S3. Crystal data and structure refinement for P2_cif

\begin{tabular}{|c|c|c|}
\hline Identification code & $\mathrm{P} 2$ & \\
\hline Empirical formula & $\mathrm{C}_{31} \mathrm{H}_{29} \mathrm{~N}_{2} \mathrm{O}$ & \\
\hline Formula weight & 445.59 & \\
\hline Temperature & $293(2) \mathrm{K}$ & \\
\hline Wavelength & $0.71073 \AA$ & \\
\hline Crystal system & Monoclinic & \\
\hline Space group & $\mathrm{C} 2 / \mathrm{c}$ & \\
\hline Unit cell dimensions & $\mathrm{a}=32.134(4) \AA$ & $\alpha=90^{\circ}$. \\
\hline & $\mathrm{b}=10.0646(11) \AA$ & $\beta=122.84^{\circ}$. \\
\hline & $\mathrm{c}=17.425(2) \AA$ & $\gamma=90^{\circ}$. \\
\hline Volume & $4735.1(10) \AA^{3}$ & \\
\hline $\bar{Z}$ & 8 & \\
\hline Density (calculated) & $1.250 \mathrm{Mg} / \mathrm{m}^{3}$ & \\
\hline Absorption coefficient & $0.075 \mathrm{~mm}^{-1}$ & \\
\hline $\mathrm{F}(000)$ & 1896 & \\
\hline Theta range for data collection & 1.508 to $28.665^{\circ}$. & \\
\hline Index ranges & $\begin{array}{c}-43<=\mathrm{h}<=43,-13<=\mathrm{k}<=13,- \\
23<=1<=23\end{array}$ & \\
\hline Reflections collected & 20434 & \\
\hline Independent reflections & $5133[\mathrm{R}($ int $)=0.0641]$ & \\
\hline Completeness to theta $=25.242^{\circ}$ & $84.7 \%$ & \\
\hline Refinement method & Full-matrix least-squares on $\mathrm{F}^{2}$ & \\
\hline Data / restraints / parameters & $5133 / 0 / 311$ & \\
\hline Goodness-of-fit on $\mathrm{F}^{2}$ & 0.850 & \\
\hline Final $\mathrm{R}$ indices [I $>2$ sigma(I)] & $\mathrm{R} 1=0.0462, \mathrm{wR} 2=0.1034$ & \\
\hline $\mathrm{R}$ indices (all data) & $\mathrm{R} 1=0.1356, \mathrm{wR} 2=0.1450$ & \\
\hline Largest diff. peak and hole & 0.285 and $-0.302 \mathrm{e} . \AA^{-3}$ & \\
\hline
\end{tabular}

\section{Atomic coordinates ( $\left.\times 10^{4}\right)$ and equivalent isotropic displacement parameters $\left(\AA^{2} \times 10^{3}\right)$} For P2_cif. U(eq) is defined as one third of the trace of the orthogonalized $U^{i j}$ tensor

\begin{tabular}{|c|c|r|r|r|}
\hline & & & & \\
$\mathrm{x}$ & $\mathrm{y}$ & $\mathrm{zq})$ & $36(1)$ \\
\hline $\mathrm{N}(2)$ & $2898(1)$ & $62(2)$ & $616(1)$ & $31(1)$ \\
\hline $\mathrm{C}(002)$ & $3073(1)$ & $-509(2)$ & $1435(2)$ & $30(1)$ \\
\hline $\mathrm{C}(003)$ & $2786(1)$ & $-1449(2)$ & $1550(2)$ & \\
\hline
\end{tabular}




\begin{tabular}{|c|c|c|c|c|}
\hline $\mathrm{O}(1)$ & $3837(1)$ & $518(2)$ & 1191(1) & $52(1)$ \\
\hline $\mathrm{C}(005)$ & $1587(1)$ & $-1032(2)$ & $-803(2)$ & $36(1)$ \\
\hline$C(006)$ & $2286(1)$ & $-1594(2)$ & $849(2)$ & $31(1)$ \\
\hline $\mathrm{C}(007)$ & $2105(1)$ & $-975(2)$ & $8(2)$ & $33(1)$ \\
\hline $\mathrm{C}(008)$ & $3913(1)$ & $502(2)$ & $2043(2)$ & $35(1)$ \\
\hline $\mathrm{C}(009)$ & $2987(1)$ & $-2291(2)$ & $2371(2)$ & $32(1)$ \\
\hline $\mathrm{C}(010)$ & $2437(1)$ & $-173(2)$ & $-75(2)$ & $35(1)$ \\
\hline $\mathrm{C}(011)$ & 1963(1) & $-2343(2)$ & $1088(2)$ & $38(1)$ \\
\hline $\mathrm{C}(012)$ & $3464(1)$ & $-2813(2)$ & $2838(2)$ & $37(1)$ \\
\hline $\mathrm{C}(013)$ & $4087(1)$ & $699(2)$ & $3773(2)$ & $38(1)$ \\
\hline $\mathrm{C}(014)$ & $3550(1)$ & $15(2)$ & $2182(2)$ & $32(1)$ \\
\hline $\mathrm{C}(015)$ & $2152(1)$ & $-2148(2)$ & 2092(2) & $40(1)$ \\
\hline$C(016)$ & $2677(1)$ & $-2622(2)$ & $2676(2)$ & $36(1)$ \\
\hline $\mathrm{C}(017)$ & $3656(1)$ & $173(2)$ & $3067(2)$ & $36(1)$ \\
\hline $\mathrm{C}(018)$ & $4359(1)$ & $972(2)$ & $2752(2)$ & $41(1)$ \\
\hline $\mathrm{C}(019)$ & $4463(1)$ & $1086(2)$ & $3638(2)$ & $38(1)$ \\
\hline $\mathrm{N}(1)$ & $4908(1)$ & $1553(2)$ & $4352(2)$ & $47(1)$ \\
\hline $\mathrm{C}(021)$ & $1279(1)$ & $-2129(2)$ & $-1002(2)$ & $44(1)$ \\
\hline $\mathrm{C}(022)$ & $2859(1)$ & $-3371(2)$ & $3456(2)$ & $45(1)$ \\
\hline $\mathrm{C}(023)$ & $1407(1)$ & $51(2)$ & $-1407(2)$ & $44(1)$ \\
\hline $\mathrm{C}(024)$ & $3637(1)$ & $-3578(2)$ & $3603(2)$ & $44(1)$ \\
\hline $\mathrm{C}(025)$ & 922(1) & 31(3) & $-2150(2)$ & $58(1)$ \\
\hline $\mathrm{C}(026)$ & $2255(1)$ & $563(3)$ & $-947(2)$ & $49(1)$ \\
\hline $\mathrm{C}(027)$ & $3340(1)$ & $-3831(2)$ & $3932(2)$ & $50(1)$ \\
\hline $\mathrm{C}(028)$ & $796(1)$ & $-2112(3)$ & $-1753(2)$ & $58(1)$ \\
\hline $\mathrm{C}(029)$ & $4951(1)$ & 2025(3) & $5180(2)$ & $56(1)$ \\
\hline $\mathrm{C}(030)$ & 5331(1) & $1745(2)$ & $4266(2)$ & $49(1)$ \\
\hline $\mathrm{C}(031)$ & 1751(1) & $1152(3)$ & $-1268(2)$ & $54(1)$ \\
\hline $\mathrm{C}(032)$ & $5360(1)$ & 3091(3) & $3918(2)$ & $62(1)$ \\
\hline $\mathrm{C}(033)$ & $617(1)$ & $-1030(4)$ & $-2316(2)$ & $66(1)$ \\
\hline $\mathrm{C}(034)$ & $4656(1)$ & $3274(3)$ & $5035(2)$ & $76(1)$ \\
\hline & & & & \\
\hline
\end{tabular}

Anisotropic displacement parameters $\left(\AA^{2} \times 10^{3}\right)$ for P2_cif. The anisotropic displacement factor exponent takes the form: $-2 \square 2\left[h^{2} a^{* 2} U^{11}+\ldots+2 h k^{*} b^{*} U^{12}\right]$

\begin{tabular}{|c|c|c|c|c|c|c|}
\hline Atom & $\mathrm{U}^{11}$ & $\mathrm{U}^{22}$ & $\mathrm{U}^{33}$ & $\mathrm{U}^{23}$ & $\mathrm{U}^{13}$ & $\mathrm{U}^{12}$ \\
\hline $\mathrm{N}(2)$ & $37(1)$ & $41(1)$ & $30(1)$ & $0(1)$ & $19(1)$ & $-6(1)$ \\
\hline $\mathrm{C}(002)$ & $33(1)$ & $33(1)$ & $32(1)$ & $-2(1)$ & $20(1)$ & $-2(1)$ \\
\hline $\mathrm{C}(003)$ & $33(1)$ & $31(1)$ & $32(1)$ & $-3(1)$ & $21(1)$ & $-2(1)$ \\
\hline $\mathrm{O}(1)$ & $52(1)$ & $73(1)$ & $39(1)$ & $-7(1)$ & $31(1)$ & $-22(1)$ \\
\hline
\end{tabular}




\begin{tabular}{|c|c|c|c|c|c|c|}
\hline $\mathrm{C}(005)$ & $33(1)$ & $45(1)$ & $31(1)$ & $-9(1)$ & $18(1)$ & $2(1)$ \\
\hline $\mathrm{C}(006)$ & $34(1)$ & 31(1) & $33(1)$ & $-6(1)$ & $22(1)$ & $-4(1)$ \\
\hline $\mathrm{C}(007)$ & $33(1)$ & $33(1)$ & $32(1)$ & $-5(1)$ & $18(1)$ & $-1(1)$ \\
\hline $\mathrm{C}(008)$ & $38(1)$ & $37(1)$ & $34(1)$ & $-2(1)$ & $22(1)$ & $-6(1)$ \\
\hline $\mathrm{C}(009)$ & $37(1)$ & $30(1)$ & $31(1)$ & $-6(1)$ & $20(1)$ & $-7(1)$ \\
\hline $\mathrm{C}(010)$ & $42(1)$ & $35(1)$ & $33(1)$ & $-2(1)$ & $23(1)$ & $-2(1)$ \\
\hline $\mathrm{C}(011)$ & $34(1)$ & $40(1)$ & $41(2)$ & $-4(1)$ & $23(1)$ & $-7(1)$ \\
\hline $\mathrm{C}(012)$ & $40(1)$ & $34(1)$ & $39(2)$ & $-3(1)$ & $23(1)$ & $-4(1)$ \\
\hline $\mathrm{C}(013)$ & $40(1)$ & $39(1)$ & $33(1)$ & $-6(1)$ & $20(1)$ & $-7(1)$ \\
\hline $\mathrm{C}(014)$ & $34(1)$ & $32(1)$ & $33(1)$ & $-3(1)$ & $19(1)$ & $-4(1)$ \\
\hline $\mathrm{C}(015)$ & $44(1)$ & $41(1)$ & $46(2)$ & $0(1)$ & $33(1)$ & $-6(1)$ \\
\hline $\mathrm{C}(016)$ & $46(1)$ & $28(1)$ & $41(2)$ & $-5(1)$ & $28(1)$ & $-7(1)$ \\
\hline $\mathrm{C}(017)$ & $38(1)$ & $35(1)$ & $40(1)$ & $-2(1)$ & $25(1)$ & $-4(1)$ \\
\hline $\mathrm{C}(018)$ & $39(1)$ & $44(1)$ & $46(2)$ & $-7(1)$ & $28(1)$ & $-11(1)$ \\
\hline $\mathrm{C}(019)$ & $36(1)$ & $34(1)$ & $41(2)$ & $-6(1)$ & 19(1) & $-5(1)$ \\
\hline $\mathrm{N}(1)$ & $37(1)$ & $57(1)$ & $44(1)$ & $-12(1)$ & $20(1)$ & $-13(1)$ \\
\hline $\mathrm{C}(021)$ & $37(1)$ & $55(2)$ & $43(2)$ & $-13(1)$ & $22(1)$ & $-6(1)$ \\
\hline $\mathrm{C}(022)$ & $60(2)$ & $40(1)$ & $47(2)$ & $2(1)$ & $36(2)$ & $-9(1)$ \\
\hline $\mathrm{C}(023)$ & $43(2)$ & $54(2)$ & $37(2)$ & $-1(1)$ & $23(1)$ & 11(1) \\
\hline $\mathrm{C}(024)$ & $45(1)$ & $35(1)$ & $42(2)$ & $3(1)$ & $17(1)$ & $-1(1)$ \\
\hline $\mathrm{C}(025)$ & $45(2)$ & $82(2)$ & $44(2)$ & $7(2)$ & $21(2)$ & $21(2)$ \\
\hline $\mathrm{C}(026)$ & $50(2)$ & $56(2)$ & $36(2)$ & $7(1)$ & $20(1)$ & $-5(1)$ \\
\hline $\mathrm{C}(027)$ & $61(2)$ & $41(1)$ & $42(2)$ & $9(1)$ & $25(2)$ & $-4(1)$ \\
\hline $\mathrm{C}(028)$ & $37(1)$ & $87(2)$ & $50(2)$ & $-21(2)$ & $23(2)$ & $-13(1)$ \\
\hline $\mathrm{C}(029)$ & $51(2)$ & $64(2)$ & $46(2)$ & $-14(1)$ & 21(2) & $-17(1)$ \\
\hline $\mathrm{C}(030)$ & $33(1)$ & $54(2)$ & $51(2)$ & $-1(1)$ & $17(1)$ & $-5(1)$ \\
\hline $\mathrm{C}(031)$ & $63(2)$ & $49(2)$ & $45(2)$ & $9(1)$ & $27(2)$ & $10(1)$ \\
\hline $\mathrm{C}(032)$ & $59(2)$ & 61(2) & $64(2)$ & $2(2)$ & $31(2)$ & $-12(1)$ \\
\hline $\mathrm{C}(033)$ & $34(2)$ & 112(3) & $43(2)$ & $-6(2)$ & $14(2)$ & $10(2)$ \\
\hline $\mathrm{C}(034)$ & $95(3)$ & $61(2)$ & $84(3)$ & $-23(2)$ & $56(2)$ & $-16(2)$ \\
\hline
\end{tabular}


SI Table S4: The energy of ground state and excited state optimized geometries of P1, P2, PBC1 and PBC2 in aniline and TEA. It is expressed in atomic unit (Hartree). The optimization of ground state with DFT and excited state with TDDFT were done with B3LYP/6-31+G* method.

\begin{tabular}{|c|c|c|c|c|c|}
\hline Molecules & $\begin{array}{l}\text { Gas Phase } \\
\left(\mathbf{S}_{\mathbf{0}}\right)\end{array}$ & Aniline $\left(\mathbf{S}_{0}\right)$ & $\operatorname{TEA}\left(\mathbf{S}_{0}\right)$ & Aniline( $\left.\mathbf{S}_{1}\right)$ & $\operatorname{TEA}\left(\mathrm{S}_{1}\right)$ \\
\hline PBC1 & -1395.7058533 & -1395.7300237 & -1395.7239093 & -1395.7094223 & -1395.7111947 \\
\hline PBC2 & -1608.3092299 & -1608.3248005 & -1608.318092 & -1608.3244734 & -1608.3145398 \\
\hline P1 & -1171.5668635 & -1171.5836567 & -1171.5801174 & -1171.568023 & -1171.5654089 \\
\hline P2 & -1384.1668438 & -1384.1859135 & -1384.1816813 & -1384.1776879 & -1384.1742624 \\
\hline
\end{tabular}

SI Table S5: Cartesian coordinates (in $\AA$ units) of the optimized ground state geometries of P1 and $\mathrm{P} 2$ in gas phase at $\mathrm{B} 3 \mathrm{LYP} / 6-31+\mathrm{G}^{*}$ level of theory.

\begin{tabular}{|c|c|c|c|c|c|c|c|}
\hline \multicolumn{4}{|c|}{ P1 (in gas phase) } & \multicolumn{4}{|c|}{ P2 (in gas phase) } \\
\hline C & 1.827873 & -0.21356 & -0.09535 & $\mathrm{C}$ & 2.950698 & -0.54307 & -0.0671 \\
\hline C & 1.40587 & -1.55183 & -0.28737 & $\mathrm{C}$ & 2.13391 & -1.63129 & -0.46068 \\
\hline $\mathrm{N}$ & 0.128268 & -1.91685 & -0.25911 & $\mathrm{~N}$ & 0.812134 & -1.5444 & -0.55788 \\
\hline C & -0.83698 & -0.98649 & -0.14234 & $\mathrm{C}$ & 0.19599 & -0.35859 & -0.3843 \\
\hline C & -0.53563 & 0.397015 & -0.24333 & $\mathrm{C}$ & 0.952515 & 0.843916 & -0.31034 \\
\hline C & 0.8326 & 0.778642 & -0.17551 & $\mathrm{C}$ & 2.352099 & 0.731257 & -0.09263 \\
\hline C & 3.282678 & 0.008433 & 0.134313 & $\mathrm{C}$ & 4.362487 & -0.85279 & 0.28948 \\
\hline C & 4.19883 & -0.87689 & -0.48227 & $\mathrm{C}$ & 4.99846 & -1.92863 & -0.37523 \\
\hline C & 3.642619 & -2.03219 & -1.28386 & $\mathrm{C}$ & 4.183905 & -2.73651 & -1.36034 \\
\hline C & 2.437976 & -2.62629 & -0.53794 & $\mathrm{C}$ & 2.77685 & -2.96011 & -0.78488 \\
\hline C & -1.56094 & 1.459597 & -0.46162 & $\mathrm{C}$ & 0.373752 & 2.204437 & -0.51514 \\
\hline C & -1.27414 & 2.783007 & -0.05084 & $\mathrm{C}$ & 1.031468 & 3.322322 & 0.049699 \\
\hline C & 0.102281 & 3.079109 & 0.496511 & C & 2.344068 & 3.090517 & 0.759767 \\
\hline C & 1.129191 & 2.26053 & -0.29517 & $\mathrm{C}$ & 3.135219 & 2.027563 & -0.01231 \\
\hline C & -2.19563 & -1.55265 & 0.108223 & C & -1.28245 & -0.44673 & -0.27853 \\
\hline C & -2.98285 & -1.15505 & 1.209404 & $\mathrm{C}$ & -2.0273 & 0.17613 & 0.742583 \\
\hline C & -4.22141 & -1.76048 & 1.452407 & C & -3.40997 & 0.003508 & 0.847861 \\
\hline C & -4.68716 & -2.76746 & 0.610916 & $\mathrm{C}$ & -4.12685 & -0.79525 & -0.06341 \\
\hline C & -3.91182 & -3.19199 & -0.47492 & C & -3.37541 & -1.45393 & -1.07099 \\
\hline C & -2.67576 & -2.59298 & -0.7059 & $\mathrm{C}$ & -2.00272 & -1.28394 & -1.15125 \\
\hline C & 3.783925 & 0.98264 & 1.015903 & C & 5.057928 & -0.20242 & 1.324493 \\
\hline
\end{tabular}




\begin{tabular}{|c|c|c|c|c|c|c|c|}
\hline$C$ & 5.1562 & 1.138081 & 1.217293 & $C$ & 6.374571 & -0.54381 & 1.637491 \\
\hline C & 6.057658 & 0.30551 & 0.550587 & C & 7.019242 & -1.56025 & 0.92934 \\
\hline C & 5.571412 & -0.70726 & -0.27968 & C & 6.319811 & -2.25697 & -0.05924 \\
\hline C & -2.78779 & 1.210151 & -1.10398 & C & -0.77313 & 2.429376 & -1.29887 \\
\hline C & -3.72856 & 2.224333 & -1.27867 & C & -1.28715 & 3.713805 & -1.47038 \\
\hline C & -3.45977 & 3.516719 & -0.82217 & C & -0.66096 & 4.807285 & -0.86688 \\
\hline C & -2.2285 & 3.789362 & -0.22326 & C & 0.501461 & 4.604492 & -0.12093 \\
\hline 0 & -2.59187 & -0.20077 & 2.10865 & 0 & -1.46143 & 0.941317 & 1.726916 \\
\hline $\mathrm{H}$ & 3.317737 & -1.69086 & -2.27887 & $\mathrm{~N}$ & -5.52293 & -0.91255 & -0.00055 \\
\hline $\mathrm{H}$ & 4.415251 & -2.79245 & -1.44373 & C & -6.13674 & -2.18626 & -0.39576 \\
\hline $\mathrm{H}$ & 1.965608 & -3.43615 & -1.10298 & C & -6.22715 & -0.1303 & 1.021066 \\
\hline $\mathrm{H}$ & 2.77307 & -3.05409 & 0.419495 & C & -7.73802 & -0.0182 & 0.803253 \\
\hline $\mathrm{H}$ & 0.319769 & 4.150219 & 0.416905 & C & -5.97424 & -3.31613 & 0.632297 \\
\hline $\mathrm{H}$ & 0.167986 & 2.819124 & 1.565333 & $\mathrm{H}$ & 4.099477 & -2.20325 & -2.31989 \\
\hline $\mathrm{H}$ & 1.043996 & 2.53544 & -1.35891 & $\mathrm{H}$ & 4.676401 & -3.69299 & -1.56845 \\
\hline $\mathrm{H}$ & 2.147355 & 2.501698 & 0.002698 & $\mathrm{H}$ & 2.129164 & -3.49707 & -1.48539 \\
\hline $\mathrm{H}$ & -4.79689 & -1.42926 & 2.311895 & $\mathrm{H}$ & 2.846026 & -3.57479 & 0.125842 \\
\hline $\mathrm{H}$ & -5.65262 & -3.22665 & 0.808175 & $\mathrm{H}$ & 2.913312 & 4.024989 & 0.822057 \\
\hline $\mathrm{H}$ & -4.26427 & -3.98512 & -1.12849 & $\mathrm{H}$ & 2.18017 & 2.747662 & 1.794233 \\
\hline $\mathrm{H}$ & -2.05228 & -2.92439 & -1.5313 & $\mathrm{H}$ & 3.2909 & 2.391423 & -1.0409 \\
\hline $\mathrm{H}$ & 3.096856 & 1.602927 & 1.583153 & $\mathrm{H}$ & 4.126027 & 1.882468 & 0.412992 \\
\hline $\mathrm{H}$ & 5.516921 & 1.898184 & 1.905577 & $\mathrm{H}$ & -3.8907 & 0.488088 & 1.687651 \\
\hline $\mathrm{H}$ & 7.128006 & 0.42263 & 0.699189 & $\mathrm{H}$ & -3.86024 & -2.08474 & -1.80615 \\
\hline $\mathrm{H}$ & 6.266414 & -1.38957 & -0.76497 & $\mathrm{H}$ & -1.44864 & -1.80498 & -1.92652 \\
\hline $\mathrm{H}$ & -3.01037 & 0.217121 & -1.47657 & $\mathrm{H}$ & 4.55597 & 0.552961 & 1.921351 \\
\hline $\mathrm{H}$ & -4.66917 & 2.002665 & -1.77606 & $\mathrm{H}$ & 6.888206 & -0.0256 & 2.443379 \\
\hline $\mathrm{H}$ & -4.19168 & 4.309974 & -0.95112 & $\mathrm{H}$ & 8.045832 & -1.82929 & 1.164411 \\
\hline $\mathrm{H}$ & -1.99508 & 4.801502 & 0.101415 & $\mathrm{H}$ & 6.8002 & -3.08095 & -0.58322 \\
\hline \multirow[t]{15}{*}{$\mathrm{H}$} & -1.74253 & 0.183075 & 1.835608 & $\mathrm{H}$ & -1.2683 & 1.593691 & -1.7802 \\
\hline & & & & $\mathrm{H}$ & -2.17544 & 3.858363 & -2.07994 \\
\hline & & & & $\mathrm{H}$ & -1.06084 & 5.810201 & -0.99318 \\
\hline & & & & $\mathrm{H}$ & 1.0172 & 5.453919 & 0.322901 \\
\hline & & & & $\mathrm{H}$ & -0.50986 & 1.038967 & 1.560526 \\
\hline & & & & $\mathrm{H}$ & -7.19718 & -2.0037 & -0.58717 \\
\hline & & & & $\mathrm{H}$ & -5.71925 & -2.49682 & -1.35565 \\
\hline & & & & $\mathrm{H}$ & -6.03265 & -0.52065 & 2.036428 \\
\hline & & & & $\mathrm{H}$ & -5.81097 & 0.880985 & 0.989989 \\
\hline & & & & $\mathrm{H}$ & -8.13993 & 0.720973 & 1.505801 \\
\hline & & & & $\mathrm{H}$ & -7.96726 & 0.321171 & -0.21361 \\
\hline & & & & $\mathrm{H}$ & -8.26957 & -0.95838 & 0.984591 \\
\hline & & & & $\mathrm{H}$ & -6.451 & -4.23422 & 0.266407 \\
\hline & & & & $\mathrm{H}$ & -4.91546 & -3.53041 & 0.815233 \\
\hline & & & & $\mathrm{H}$ & -6.43873 & -3.05649 & 1.591136 \\
\hline
\end{tabular}


SI Table S6: Cartesian coordinates (in $\AA$ units) of the optimized ground state geometries of P1 and $\mathrm{P} 2$ in aniline at B3LYP/6-31+G* level of theory.

\begin{tabular}{|c|c|c|c|c|c|c|c|}
\hline \multicolumn{4}{|c|}{ P1 (in aniline) } & \multicolumn{4}{|c|}{ P2 (in aniline) } \\
\hline C & 1.828831 & -0.21586 & -0.09979 & C & 2.958169 & -0.54665 & -0.06302 \\
\hline C & 1.409877 & -1.55705 & -0.27859 & $\mathrm{C}$ & 2.143343 & -1.64957 & -0.41754 \\
\hline$N$ & 0.13036 & -1.92278 & -0.24699 & $\mathrm{~N}$ & 0.818134 & -1.5688 & -0.50596 \\
\hline C & -0.83464 & -0.98838 & -0.14582 & $\mathrm{C}$ & 0.202078 & -0.37688 & -0.36675 \\
\hline C & -0.5345 & 0.392524 & -0.25348 & $\mathrm{C}$ & 0.954214 & 0.827578 & -0.3269 \\
\hline C & 0.833777 & 0.775999 & -0.18665 & $\mathrm{C}$ & 2.356474 & 0.725357 & -0.11737 \\
\hline C & 3.284332 & 0.009987 & 0.127356 & $\mathrm{C}$ & 4.374848 & -0.8412 & 0.290884 \\
\hline C & 4.200949 & -0.87961 & -0.48315 & $\mathrm{C}$ & 5.009416 & -1.93295 & -0.34947 \\
\hline C & 3.64639 & -2.04354 & -1.27276 & $\mathrm{C}$ & 4.190047 & -2.77264 & -1.30316 \\
\hline C & 2.444346 & -2.63159 & -0.5179 & $\mathrm{C}$ & 2.789826 & -2.98454 & -0.70701 \\
\hline C & -1.56234 & 1.453835 & -0.46477 & $\mathrm{C}$ & 0.365976 & 2.181755 & -0.54739 \\
\hline C & -1.27629 & 2.775232 & -0.0456 & $\mathrm{C}$ & 1.02373 & 3.310955 & -0.00447 \\
\hline C & 0.101639 & 3.071007 & 0.496848 & $\mathrm{C}$ & 2.344069 & 3.096735 & 0.695889 \\
\hline C & 1.126059 & 2.258158 & -0.30434 & $\mathrm{C}$ & 3.132821 & 2.026429 & -0.06856 \\
\hline C & -2.1977 & -1.54867 & 0.102743 & $\mathrm{C}$ & -1.27756 & -0.45669 & -0.25084 \\
\hline C & -2.9636 & -1.17241 & 1.225508 & $C$ & -2.0016 & 0.150416 & 0.793674 \\
\hline C & -4.20995 & -1.76193 & 1.468008 & $C$ & -3.38465 & 0.001801 & 0.914479 \\
\hline$C$ & -4.70543 & -2.73555 & 0.602416 & $C$ & -4.13302 & -0.75756 & -0.01232 \\
\hline C & -3.95178 & -3.13932 & -0.50663 & $C$ & -3.39942 & -1.40724 & -1.04265 \\
\hline C & -2.70826 & -2.55286 & -0.73742 & $C$ & -2.02389 & -1.25651 & -1.13545 \\
\hline C & 3.784051 & 0.992801 & 1.001124 & $C$ & 5.074699 & -0.15882 & 1.30275 \\
\hline$C$ & 5.157057 & 1.15163 & 1.199707 & $C$ & 6.395719 & -0.48694 & 1.614689 \\
\hline C & 6.059558 & 0.314685 & 0.53817 & $C$ & 7.039357 & -1.52036 & 0.928821 \\
\hline$C$ & 5.574264 & -0.70617 & -0.28395 & $C$ & 6.335432 & -2.24733 & -0.03553 \\
\hline$C$ & -2.78971 & 1.206444 & -1.10809 & $C$ & -0.78781 & 2.389962 & -1.32652 \\
\hline C & -3.73459 & 2.219798 & -1.27308 & $C$ & -1.31123 & 3.66969 & -1.51242 \\
\hline$C$ & -3.46791 & 3.509418 & -0.805 & C & -0.68595 & 4.774972 & -0.92775 \\
\hline C & -2.23482 & 3.780529 & -0.20706 & $C$ & 0.484521 & 4.588339 & -0.18858 \\
\hline $\mathrm{O}$ & -2.54128 & -0.24741 & 2.145359 & $\mathrm{O}$ & -1.40403 & 0.887043 & 1.787126 \\
\hline $\mathrm{H}$ & 3.319281 & -1.71389 & -2.27063 & $\mathrm{~N}$ & -5.52221 & -0.84537 & 0.068348 \\
\hline $\mathrm{H}$ & 4.419007 & -2.80464 & -1.4245 & $C$ & -6.2064 & -1.97897 & -0.56638 \\
\hline $\mathrm{H}$ & 1.977891 & -3.44978 & -1.0758 & $C$ & -6.18826 & -0.19843 & 1.209331 \\
\hline $\mathrm{H}$ & 2.782787 & -3.04777 & 0.443442 & $C$ & -7.70932 & -0.08999 & 1.094134 \\
\hline $\mathrm{H}$ & 0.317632 & 4.142043 & 0.420719 & $\mathrm{C}$ & -6.0688 & -3.30904 & 0.188382 \\
\hline $\mathrm{H}$ & 0.17178 & 2.804508 & 1.563031 & $\mathrm{H}$ & 4.094135 & -2.26914 & -2.27725 \\
\hline $\mathrm{H}$ & 1.035795 & 2.535274 & -1.3669 & $\mathrm{H}$ & 4.683276 & -3.73314 & -1.48627 \\
\hline $\mathrm{H}$ & 2.14362 & 2.502951 & -0.00941 & $\mathrm{H}$ & 2.142327 & -3.54755 & -1.38687 \\
\hline
\end{tabular}




\begin{tabular}{|c|c|c|c|c|c|c|c|}
\hline $\mathrm{H}$ & -4.77138 & -1.44971 & 2.344094 & $\mathrm{H}$ & 2.871479 & -3.57145 & 0.220821 \\
\hline $\mathrm{H}$ & -5.6761 & -3.18337 & 0.799084 & $\mathrm{H}$ & 2.909499 & 4.03389 & 0.738057 \\
\hline $\mathrm{H}$ & -4.32773 & -3.90377 & -1.18084 & $\mathrm{H}$ & 2.190885 & 2.767269 & 1.735553 \\
\hline $\mathrm{H}$ & -2.10894 & -2.86328 & -1.5888 & $\mathrm{H}$ & 3.275425 & 2.372432 & -1.10503 \\
\hline $\mathrm{H}$ & 3.098449 & 1.619071 & 1.563002 & $\mathrm{H}$ & 4.128283 & 1.896452 & 0.349412 \\
\hline $\mathrm{H}$ & 5.516948 & 1.918594 & 1.880613 & $\mathrm{H}$ & -3.84696 & 0.477277 & 1.769407 \\
\hline $\mathrm{H}$ & 7.129856 & 0.434859 & 0.684535 & $\mathrm{H}$ & -3.89669 & -2.0267 & -1.77807 \\
\hline $\mathrm{H}$ & 6.269681 & -1.3915 & -0.76396 & $\mathrm{H}$ & -1.49299 & -1.7665 & -1.93457 \\
\hline $\mathrm{H}$ & -3.00926 & 0.217535 & -1.49334 & $\mathrm{H}$ & 4.576651 & 0.612648 & 1.881666 \\
\hline $\mathrm{H}$ & -4.67441 & 2.000825 & -1.77321 & $\mathrm{H}$ & 6.913429 & 0.055928 & 2.401339 \\
\hline $\mathrm{H}$ & -4.20163 & 4.302124 & -0.92655 & $\mathrm{H}$ & 8.068883 & -1.77856 & 1.163033 \\
\hline $\mathrm{H}$ & -2.00264 & 4.790756 & 0.123659 & $\mathrm{H}$ & 6.815021 & -3.08365 & -0.5399 \\
\hline \multirow[t]{15}{*}{$\mathrm{H}$} & -1.67065 & 0.1033 & 1.890983 & $\mathrm{H}$ & -1.2803 & 1.546316 & -1.7966 \\
\hline & & & & $\mathrm{H}$ & -2.2034 & 3.801557 & -2.11923 \\
\hline & & & & $\mathrm{H}$ & -1.09158 & 5.77405 & -1.06529 \\
\hline & & & & $\mathrm{H}$ & 0.999937 & 5.446654 & 0.237603 \\
\hline & & & & $\mathrm{H}$ & -0.44645 & 0.93903 & 1.62798 \\
\hline & & & & $\mathrm{H}$ & -7.26142 & -1.71787 & -0.66729 \\
\hline & & & & $\mathrm{H}$ & -5.83827 & -2.08538 & -1.58954 \\
\hline & & & & $\mathrm{H}$ & -5.92876 & -0.70652 & 2.154122 \\
\hline & & & & $\mathrm{H}$ & -5.7921 & 0.818914 & 1.280576 \\
\hline & & & & $\mathrm{H}$ & -8.07142 & 0.536117 & 1.917381 \\
\hline & & & & $\mathrm{H}$ & -8.00802 & 0.386214 & 0.153102 \\
\hline & & & & $\mathrm{H}$ & -8.21692 & -1.0567 & 1.173685 \\
\hline & & & & $\mathrm{H}$ & -6.60691 & -4.10231 & -0.34514 \\
\hline & & & & $\mathrm{H}$ & -5.01885 & -3.60933 & 0.276976 \\
\hline & & & & $\mathrm{H}$ & -6.48841 & -3.23723 & 1.198837 \\
\hline
\end{tabular}

SI Table S7: Cartesian coordinates (in $\AA$ units) of the optimized ground state geometries of P1 and $\mathrm{P} 2$ in TEA at B3LYP/6-31+G* level of theory.

\begin{tabular}{|c|c|c|c|c|c|c|c|}
\hline \multicolumn{4}{|c|}{ P1 (in TEA) } & \multicolumn{4}{|c|}{$\mathrm{P} 2$ (in TEA) } \\
\hline C & 1.828064 & -0.21475 & -0.09893 & $\mathrm{C}$ & 2.953095 & -0.54887 & -0.06265 \\
\hline C & 1.407866 & -1.55454 & -0.28466 & $\mathrm{C}$ & 2.134723 & -1.64478 & -0.43079 \\
\hline$N$ & 0.129173 & -1.91999 & -0.25444 & $\mathrm{~N}$ & 0.811194 & -1.55854 & -0.52345 \\
\hline C & -0.83583 & -0.98746 & -0.14516 & $\mathrm{C}$ & 0.198094 & -0.36728 & -0.3723 \\
\hline C & -0.53548 & 0.394901 & -0.24893 & C & 0.955099 & 0.835006 & -0.32233 \\
\hline C & 0.832929 & 0.777346 & -0.18221 & $\mathrm{C}$ & 2.356033 & 0.725609 & -0.1094 \\
\hline C & 3.283166 & 0.009276 & 0.13011 & $\mathrm{C}$ & 4.366697 & -0.85222 & 0.294346 \\
\hline C & 4.199847 & -0.87791 & -0.4833 & $\mathrm{C}$ & 4.999548 & -1.94107 & -0.35225 \\
\hline C & 3.644886 & -2.03713 & -1.27974 & $\mathrm{C}$ & 4.180707 & -2.76855 & -1.31715 \\
\hline C & 2.441265 & -2.6287 & -0.53012 & C & 2.776532 & -2.97985 & -0.73016 \\
\hline
\end{tabular}




\begin{tabular}{|c|c|c|c|c|c|c|c|}
\hline $\mathrm{C}$ & -1.56218 & 1.457039 & -0.46276 & $\mathrm{C}$ & 0.374239 & 2.192455 & -0.54217 \\
\hline$C$ & -1.27493 & 2.779562 & -0.04863 & $\mathrm{C}$ & 1.035811 & 3.317933 & 0.003331 \\
\hline$C$ & 0.10262 & 3.075471 & 0.49524 & C & 2.352181 & 3.096004 & 0.709107 \\
\hline$C$ & 1.127717 & 2.259222 & -0.30126 & C & 3.139009 & 2.022809 & -0.05315 \\
\hline$C$ & -2.19677 & -1.5508 & 0.104935 & $\mathrm{C}$ & -1.28083 & -0.4461 & -0.25731 \\
\hline$C$ & -2.97029 & -1.16707 & 1.220127 & $\mathrm{C}$ & -2.00902 & 0.171069 & 0.778934 \\
\hline$C$ & -4.21358 & -1.76264 & 1.462839 & C & -3.39129 & 0.01514 & 0.899383 \\
\hline$C$ & -4.69781 & -2.74888 & 0.606135 & C & -4.13286 & -0.76 & -0.01774 \\
\hline$C$ & -3.93592 & -3.16071 & -0.49414 & C & -3.39569 & -1.41821 & -1.03898 \\
\hline$C$ & -2.69533 & -2.56937 & -0.72505 & $\mathrm{C}$ & -2.02114 & -1.26246 & -1.13157 \\
\hline$C$ & 3.783036 & 0.987246 & 1.008773 & $\mathrm{C}$ & 5.065735 & -0.18177 & 1.314379 \\
\hline$C$ & 5.155556 & 1.143998 & 1.210036 & C & 6.383813 & -0.51774 & 1.628928 \\
\hline$C$ & 6.057947 & 0.30952 & 0.546038 & $\mathrm{C}$ & 7.025746 & -1.54797 & 0.937476 \\
\hline C & 5.572695 & -0.70664 & -0.2813 & $\mathrm{C}$ & 6.322529 & -2.26366 & -0.0353 \\
\hline C & -2.79005 & 1.208911 & -1.10423 & $\mathrm{C}$ & -0.77684 & 2.406934 & -1.32313 \\
\hline$C$ & -3.73273 & 2.222944 & -1.27352 & C & -1.29221 & 3.689254 & -1.50998 \\
\hline$C$ & -3.4641 & 3.514034 & -0.812 & 6 & -0.66227 & 4.790968 & -0.92461 \\
\hline$C$ & -2.23117 & 3.785665 & -0.21497 & 6 & 0.504673 & 4.598026 & -0.18225 \\
\hline 0 & -2.55894 & -0.23201 & 2.133069 & 0 & -1.41891 & 0.920915 & 1.764595 \\
\hline $\mathrm{H}$ & 3.319361 & -1.7012 & -2.27618 & $\mathrm{~N}$ & -5.52351 & -0.85586 & 0.062829 \\
\hline $\mathrm{H}$ & 4.417554 & -2.79777 & -1.43542 & C & -6.19587 & -2.00942 & -0.54538 \\
\hline $\mathrm{H}$ & 1.97232 & -3.44234 & -1.0925 & C & -6.19386 & -0.19478 & 1.19123 \\
\hline $\mathrm{H}$ & 2.777547 & -3.05149 & 0.42908 & $\mathrm{C}$ & -7.71504 & -0.0912 & 1.069094 \\
\hline $\mathrm{H}$ & 0.319597 & 4.146421 & 0.416631 & C & -6.04291 & -3.32227 & 0.236791 \\
\hline $\mathrm{H}$ & 0.170785 & 2.812882 & 1.562904 & $\mathrm{H}$ & 4.091887 & -2.25544 & -2.28704 \\
\hline $\mathrm{H}$ & 1.039906 & 2.534898 & -1.36447 & $\mathrm{H}$ & 4.671315 & -3.72938 & -1.50714 \\
\hline $\mathrm{H}$ & 2.145742 & 2.501935 & -0.00523 & $\mathrm{H}$ & 2.127849 & -3.53248 & -1.41732 \\
\hline $\mathrm{H}$ & -4.77999 & -1.44321 & 2.332919 & $\mathrm{H}$ & 2.850404 & -3.57555 & 0.192696 \\
\hline $\mathrm{H}$ & -5.66646 & -3.20118 & 0.803107 & $\mathrm{H}$ & 2.921906 & 4.030702 & 0.755457 \\
\hline $\mathrm{H}$ & -4.30254 & -3.93685 & -1.16016 & $\mathrm{H}$ & 2.193147 & 2.766474 & 1.748279 \\
\hline $\mathrm{H}$ & -2.08612 & -2.88863 & -1.56594 & $\mathrm{H}$ & 3.28839 & 2.370973 & -1.08798 \\
\hline $\mathrm{H}$ & 3.096372 & 1.610476 & 1.573044 & $\mathrm{H}$ & 4.132105 & 1.885404 & 0.368724 \\
\hline $\mathrm{H}$ & 5.515485 & 1.906934 & 1.895526 & $\mathrm{H}$ & -3.85566 & 0.499157 & 1.748223 \\
\hline $\mathrm{H}$ & 7.128167 & 0.427822 & 0.694581 & $\mathrm{H}$ & -3.88896 & -2.0495 & -1.76708 \\
\hline $\mathrm{H}$ & 6.268153 & -1.39025 & -0.7639 & $\mathrm{H}$ & -1.48411 & -1.78358 & -1.91907 \\
\hline $\mathrm{H}$ & -3.01215 & 0.217983 & -1.48259 & $\mathrm{H}$ & 4.567482 & 0.586063 & 1.898145 \\
\hline $\mathrm{H}$ & -4.67364 & 2.002597 & -1.77093 & $\mathrm{H}$ & 6.900581 & 0.015994 & 2.422508 \\
\hline $\mathrm{H}$ & -4.19682 & 4.307209 & -0.93666 & $\mathrm{H}$ & 8.053191 & -1.81261 & 1.173741 \\
\hline $\mathrm{H}$ & -1.99772 & 4.796857 & 0.11227 & $\mathrm{H}$ & 6.800633 & -3.0978 & -0.54488 \\
\hline \multirow[t]{4}{*}{$\mathrm{H}$} & -1.69674 & 0.131634 & 1.870441 & $\mathrm{H}$ & -1.27418 & 1.565473 & -1.79196 \\
\hline & & & & $\mathrm{H}$ & -2.18329 & 3.825598 & -2.11737 \\
\hline & & & & $\mathrm{H}$ & -1.0624 & 5.792206 & -1.06284 \\
\hline & & & & $\mathrm{H}$ & 1.023235 & 5.453458 & 0.246223 \\
\hline
\end{tabular}




\begin{tabular}{|l|rrrr|}
\hline & H & -0.46537 & 0.995243 & 1.59355 \\
H & -7.25403 & -1.76195 & -0.65126 \\
H & -5.82767 & -2.13383 & -1.56664 \\
H & -5.93666 & -0.68686 & 2.145602 \\
H & -5.80024 & 0.824464 & 1.247951 \\
H & -8.08273 & 0.548387 & 1.879542 \\
H & -8.01056 & 0.36759 & 0.118419 \\
H & -8.22182 & -1.05721 & 1.163581 \\
& H & -6.5708 & -4.13398 & -0.27929 \\
H & -4.98932 & -3.60668 & 0.332414 \\
\hline & -6.46336 & -3.23496 & 1.245804 \\
\hline
\end{tabular}

SI Table S8: Cartesian coordinates (in $\AA$ units) of the optimized ground state geometries of PBC1 and PBC2 in aniline at B3LYP/6-31+G* level of theory

\begin{tabular}{|c|c|c|c|c|c|c|c|}
\hline \multicolumn{4}{|c|}{ PBC1 (in aniline) } & \multicolumn{4}{|c|}{ PBC2 (in aniline) } \\
\hline C & -1.78988 & 0.167502 & 0.020757 & $\mathrm{C}$ & -2.93084 & -0.23762 & -0.137 \\
\hline C & -1.3306 & -1.16351 & 0.092602 & $\mathrm{C}$ & -2.16035 & -1.38464 & 0.132085 \\
\hline$N$ & -0.00708 & -1.44935 & 0.12829 & $\mathrm{~N}$ & -0.82651 & -1.30269 & 0.37283 \\
\hline C & 0.94109 & -0.4634 & 0.007005 & $\mathrm{C}$ & -0.14324 & -0.10955 & 0.215236 \\
\hline C & 0.54461 & 0.887702 & 0.037806 & $\mathrm{C}$ & -0.89159 & 1.089924 & 0.115142 \\
\hline C & -0.83229 & 1.199516 & -0.06629 & $\mathrm{C}$ & -2.27046 & 1.013667 & -0.17288 \\
\hline C & -3.26324 & 0.388822 & 0.053449 & $\mathrm{C}$ & -4.40119 & -0.41771 & -0.30586 \\
\hline C & -4.10832 & -0.61289 & -0.47646 & $\mathrm{C}$ & -4.87491 & -1.64059 & -0.83326 \\
\hline C & -3.46537 & -1.88818 & -0.95614 & $\mathrm{C}$ & -3.85849 & -2.71623 & -1.10621 \\
\hline C & -2.34175 & -2.28546 & 0.01431 & $\mathrm{C}$ & -2.82248 & -2.74262 & 0.028964 \\
\hline C & 1.494299 & 2.020407 & 0.226008 & $\mathrm{C}$ & -0.32027 & 2.440816 & 0.373461 \\
\hline C & 1.201235 & 3.249579 & -0.39851 & $\mathrm{C}$ & -0.8434 & 3.541052 & -0.3347 \\
\hline C & -0.08708 & 3.330928 & -1.17504 & C & -1.97316 & 3.255082 & -1.28832 \\
\hline C & -1.19302 & 2.643149 & -0.35735 & $\mathrm{C}$ & -2.95863 & 2.297893 & -0.59798 \\
\hline C & 2.320629 & -0.92059 & -0.25163 & $\mathrm{C}$ & 1.303775 & -0.21235 & 0.040937 \\
\hline C & 2.733447 & -2.16434 & 0.2674 & $\mathrm{C}$ & 1.989596 & -1.41923 & 0.32729 \\
\hline C & 4.044996 & -2.61978 & 0.084249 & C & 3.353014 & -1.57901 & 0.071057 \\
\hline C & 4.932679 & -1.87223 & -0.68506 & $\mathrm{C}$ & 4.117347 & -0.56357 & -0.5424 \\
\hline C & 4.512979 & -0.675 & -1.28511 & C & 3.419951 & 0.636268 & -0.88189 \\
\hline C & 3.221784 & -0.20662 & -1.06676 & $\mathrm{C}$ & 2.077932 & 0.785713 & -0.60363 \\
\hline C & -3.85213 & 1.517994 & 0.650773 & $\mathrm{C}$ & -5.34622 & 0.539132 & 0.108765 \\
\hline C & -5.2379 & 1.687089 & 0.658401 & $\mathrm{C}$ & -6.71507 & 0.324732 & -0.06658 \\
\hline C & -6.06328 & 0.722143 & 0.075822 & $\mathrm{C}$ & -7.16991 & -0.86074 & -0.64867 \\
\hline C & -5.49359 & -0.42933 & -0.47402 & $\mathrm{C}$ & -6.24561 & -1.84366 & -1.01287 \\
\hline C & 2.597965 & 1.939285 & 1.092863 & $\mathrm{C}$ & 0.624002 & 2.668869 & 1.390449 \\
\hline C & 3.433298 & 3.039508 & 1.283615 & $\mathrm{C}$ & 1.086696 & 3.957488 & 1.653876 \\
\hline
\end{tabular}




\begin{tabular}{|c|c|c|c|c|c|c|c|}
\hline$C$ & 3.173291 & 4.241125 & 0.615189 & $\mathrm{C}$ & 0.605572 & 5.041504 & 0.910122 \\
\hline$C$ & 2.051666 & 4.344212 & -0.2112 & C & -0.36613 & 4.829435 & -0.07116 \\
\hline 0 & 1.852665 & -2.91283 & 0.966121 & 0 & 1.343795 & -2.47982 & 0.842897 \\
\hline B & 0.498432 & -2.96561 & 0.477358 & B & -0.01396 & -2.37295 & 1.302006 \\
\hline $\mathrm{F}$ & -0.31147 & -3.47156 & 1.492326 & $\mathrm{~F}$ & -0.09262 & -1.81036 & 2.595923 \\
\hline $\mathrm{F}$ & 0.383463 & -3.72561 & -0.69972 & $\mathrm{~F}$ & -0.5794 & -3.64047 & 1.309885 \\
\hline $\mathrm{H}$ & -4.20385 & -2.69416 & -1.01323 & $\mathrm{~N}$ & 5.457064 & -0.73468 & -0.83367 \\
\hline $\mathrm{H}$ & -3.04783 & -1.76279 & -1.96627 & $\mathrm{C}$ & 6.29585 & 0.427635 & -1.15269 \\
\hline $\mathrm{H}$ & -2.76457 & -2.47633 & 1.009817 & $\mathrm{C}$ & 6.114118 & -1.97683 & -0.38704 \\
\hline $\mathrm{H}$ & -1.86929 & -3.20691 & -0.31955 & $\mathrm{C}$ & 7.516467 & -2.21412 & -0.94618 \\
\hline $\mathrm{H}$ & 0.007049 & 2.833001 & -2.15137 & $\mathrm{C}$ & 6.71447 & 1.255562 & 0.070259 \\
\hline $\mathrm{H}$ & -0.36031 & 4.372955 & -1.36968 & $\mathrm{H}$ & -4.34246 & -3.69518 & -1.18473 \\
\hline $\mathrm{H}$ & -2.14318 & 2.683418 & -0.89592 & $\mathrm{H}$ & -3.34234 & -2.53561 & -2.06125 \\
\hline $\mathrm{H}$ & -1.33249 & 3.200332 & 0.580273 & $\mathrm{H}$ & -3.31582 & -3.00314 & 0.976052 \\
\hline $\mathrm{H}$ & 4.333713 & -3.5692 & 0.525245 & $\mathrm{H}$ & -2.07833 & -3.50947 & -0.16749 \\
\hline $\mathrm{H}$ & 5.945418 & -2.23424 & -0.84173 & $\mathrm{H}$ & -1.60073 & 2.789071 & -2.21266 \\
\hline $\mathrm{H}$ & 5.191022 & -0.11317 & -1.92088 & $\mathrm{H}$ & -2.4869 & 4.178197 & -1.57576 \\
\hline $\mathrm{H}$ & 2.904417 & 0.718203 & -1.53545 & $\mathrm{H}$ & -3.78874 & 2.062955 & -1.26931 \\
\hline $\mathrm{H}$ & -3.23514 & 2.260658 & 1.142544 & $\mathrm{H}$ & -3.38918 & 2.810474 & 0.274342 \\
\hline $\mathrm{H}$ & -5.66862 & 2.567077 & 1.12844 & $\mathrm{H}$ & 3.783987 & -2.5261 & 0.365959 \\
\hline $\mathrm{H}$ & -7.14246 & 0.850451 & 0.074406 & $\mathrm{H}$ & 3.92515 & 1.449743 & -1.38564 \\
\hline $\mathrm{H}$ & -6.1319 & -1.20581 & -0.88962 & $\mathrm{H}$ & 1.6043 & 1.704653 & -0.92347 \\
\hline $\mathrm{H}$ & 2.803474 & 1.015091 & 1.623678 & $\mathrm{H}$ & -5.0248 & 1.447365 & 0.603646 \\
\hline $\mathrm{H}$ & 4.282658 & 2.959934 & 1.956779 & $\mathrm{H}$ & -7.42236 & 1.080621 & 0.264379 \\
\hline $\mathrm{H}$ & 3.825665 & 5.098798 & 0.756808 & $\mathrm{H}$ & -8.23376 & -1.0317 & -0.79092 \\
\hline \multirow[t]{15}{*}{$\mathrm{H}$} & 1.82119 & 5.287389 & -0.70164 & $\mathrm{H}$ & -6.59162 & -2.78937 & -1.42438 \\
\hline & & & & $\mathrm{H}$ & 0.99858 & 1.834616 & 1.975771 \\
\hline & & & & $\mathrm{H}$ & 1.81722 & 4.116145 & 2.442794 \\
\hline & & & & $\mathrm{H}$ & 0.967992 & 6.046634 & 1.109567 \\
\hline & & & & $\mathrm{H}$ & -0.77243 & 5.671967 & -0.62663 \\
\hline & & & & $\mathrm{H}$ & 5.770309 & 1.055533 & -1.8759 \\
\hline & & & & $\mathrm{H}$ & 7.18208 & 0.065918 & -1.67579 \\
\hline & & & & $\mathrm{H}$ & 6.148923 & -2.01134 & 0.71391 \\
\hline & & & & $\mathrm{H}$ & 5.485383 & -2.81234 & -0.70946 \\
\hline & & & & $\mathrm{H}$ & 7.840576 & -3.21275 & -0.6328 \\
\hline & & & & $\mathrm{H}$ & 7.526835 & -2.18827 & -2.04171 \\
\hline & & & & $\mathrm{H}$ & 8.255198 & -1.49898 & -0.57037 \\
\hline & & & & $\mathrm{H}$ & 7.345515 & 2.095902 & -0.24391 \\
\hline & & & & $\mathrm{H}$ & 7.287464 & 0.648308 & 0.780605 \\
\hline & & & & $\mathrm{H}$ & 5.842588 & 1.660726 & 0.595506 \\
\hline
\end{tabular}


SI Table S9: Cartesian coordinates (in $\AA$ units) of the optimized ground state geometries of $\mathrm{PBC} 1$ and $\mathrm{PBC} 2$ in TEA at $\mathrm{B} 3 \mathrm{LYP} / 6-31+\mathrm{G}^{*}$ level of theory.

\begin{tabular}{|c|c|c|c|c|c|c|c|}
\hline \multicolumn{4}{|c|}{ PBC1 (in TEA) } & \multicolumn{4}{|c|}{ PBC2 (in TEA) } \\
\hline C & -1.78898 & 0.169194 & 0.019065 & $\mathrm{C}$ & -2.93051 & -0.23901 & -0.13759 \\
\hline C & -1.32936 & -1.16187 & 0.092 & $\mathrm{C}$ & -2.15701 & -1.38355 & 0.137051 \\
\hline$N$ & -0.00702 & -1.44815 & 0.127002 & $\mathrm{~N}$ & -0.82452 & -1.29948 & 0.374627 \\
\hline C & 0.941746 & -0.46473 & 0.004022 & $\mathrm{C}$ & -0.1428 & -0.10777 & 0.211571 \\
\hline C & 0.546 & 0.88816 & 0.034303 & $\mathrm{C}$ & -0.89279 & 1.091423 & 0.108994 \\
\hline C & -0.83044 & 1.200437 & -0.06757 & $\mathrm{C}$ & -2.27165 & 1.01272 & -0.17657 \\
\hline C & -3.26221 & 0.390589 & 0.052585 & C & -4.40021 & -0.42301 & -0.30575 \\
\hline C & -4.10759 & -0.61153 & -0.47596 & C & -4.8715 & -1.64986 & -0.82618 \\
\hline C & -3.46444 & -1.88724 & -0.95449 & $\mathrm{C}$ & -3.85318 & -2.72614 & -1.09031 \\
\hline C & -2.33993 & -2.28457 & 0.015037 & C & -2.81768 & -2.74297 & 0.045431 \\
\hline C & 1.495786 & 2.021014 & 0.223186 & C & -0.32368 & 2.443242 & 0.366554 \\
\hline C & 1.202325 & 3.251305 & -0.39882 & C & -0.84881 & 3.542792 & -0.34094 \\
\hline C & -0.08662 & 3.333267 & -1.17454 & $\mathrm{C}$ & -1.97897 & 3.254329 & -1.29357 \\
\hline C & -1.19171 & 2.645139 & -0.35619 & C & -2.9625 & 2.296234 & -0.60165 \\
\hline C & 2.320372 & -0.92344 & -0.25225 & C & 1.303744 & -0.21054 & 0.036239 \\
\hline C & 2.729511 & -2.16998 & 0.265768 & C & 1.988662 & -1.41971 & 0.320449 \\
\hline C & 4.042917 & -2.6237 & 0.086017 & C & 3.353049 & -1.57658 & 0.061384 \\
\hline C & 4.932515 & -1.87553 & -0.67876 & C & 4.115019 & -0.56069 & -0.5493 \\
\hline$C$ & 4.516104 & -0.67639 & -1.27792 & C & 3.418848 & 0.63981 & -0.88616 \\
\hline$C$ & 3.225185 & -0.20802 & -1.06249 & C & 2.077124 & 0.788682 & -0.60659 \\
\hline C & -3.85156 & 1.519027 & 0.650481 & C & -5.34821 & 0.533306 & 0.102852 \\
\hline$C$ & -5.23715 & 1.687859 & 0.659434 & C & -6.71625 & 0.315432 & -0.0725 \\
\hline$C$ & -6.06251 & 0.722878 & 0.077774 & $C$ & -7.16814 & -0.87363 & -0.64855 \\
\hline$C$ & -5.49266 & -0.42816 & -0.47206 & $\mathrm{C}$ & -6.2414 & -1.85631 & -1.00584 \\
\hline$C$ & 2.600277 & 1.939495 & 1.088671 & $\mathrm{C}$ & 0.620464 & 2.672937 & 1.383074 \\
\hline C & 3.434506 & 3.039984 & 1.280956 & $\mathrm{C}$ & 1.080374 & 3.962352 & 1.646171 \\
\hline$C$ & 3.173466 & 4.242628 & 0.615618 & $C$ & 0.597097 & 5.045625 & 0.903346 \\
\hline$C$ & 2.051594 & 4.346227 & -0.20989 & $C$ & -0.37432 & 4.831909 & -0.07749 \\
\hline 0 & 1.851469 & -2.91849 & 0.960934 & 0 & 1.347673 & -2.47993 & 0.83249 \\
\hline B & 0.496007 & -2.97649 & 0.477002 & B & -0.00264 & -2.37113 & 1.315313 \\
\hline $\mathrm{F}$ & -0.31642 & -3.46711 & 1.491724 & $\mathrm{~F}$ & -0.06854 & -1.78603 & 2.592482 \\
\hline $\mathrm{F}$ & 0.373465 & -3.7234 & -0.70236 & $\mathrm{~F}$ & -0.57686 & -3.63005 & 1.330553 \\
\hline $\mathrm{H}$ & -4.2033 & -2.6932 & -1.01126 & $\mathrm{~N}$ & 5.457527 & -0.73067 & -0.84311 \\
\hline $\mathrm{H}$ & -3.04756 & -1.76294 & -1.96522 & C & 6.300085 & 0.437317 & -1.12395 \\
\hline $\mathrm{H}$ & -2.76029 & -2.4763 & 1.011339 & C & 6.110492 & -1.97743 & -0.40832 \\
\hline $\mathrm{H}$ & -1.86593 & -3.20529 & -0.31859 & C & 7.508446 & -2.21677 & -0.97839 \\
\hline $\mathrm{H}$ & 0.006137 & 2.835328 & -2.15123 & $C$ & 6.70761 & 1.235656 & 0.122626 \\
\hline $\mathrm{H}$ & -0.35939 & 4.375689 & -1.36947 & $\mathrm{H}$ & -4.33639 & -3.70624 & -1.16251 \\
\hline $\mathrm{H}$ & -2.14331 & 2.685897 & -0.89258 & $\mathrm{H}$ & -3.33698 & -2.55205 & -2.04681 \\
\hline
\end{tabular}




\begin{tabular}{|c|c|c|c|c|c|c|c|}
\hline $\mathrm{H}$ & -1.32886 & 3.202165 & 0.582103 & $\mathrm{H}$ & -3.31059 & -2.99497 & 0.994974 \\
\hline $\mathrm{H}$ & 4.327554 & -3.57466 & 0.525894 & $\mathrm{H}$ & -2.07196 & -3.51091 & -0.1407 \\
\hline $\mathrm{H}$ & 5.945477 & -2.23809 & -0.83369 & $\mathrm{H}$ & -1.60687 & 2.787472 & -2.21785 \\
\hline $\mathrm{H}$ & 5.196559 & -0.11397 & -1.91068 & $\mathrm{H}$ & -2.49378 & 4.176864 & -1.58226 \\
\hline $\mathrm{H}$ & 2.910398 & 0.718506 & -1.5297 & $\mathrm{H}$ & -3.79373 & 2.059561 & -1.27126 \\
\hline $\mathrm{H}$ & -3.23402 & 2.260695 & 1.143372 & $\mathrm{H}$ & -3.39186 & 2.809543 & 0.271091 \\
\hline $\mathrm{H}$ & -5.66802 & 2.56711 & 1.130867 & $\mathrm{H}$ & 3.782952 & -2.52386 & 0.356691 \\
\hline $\mathrm{H}$ & -7.14179 & 0.850532 & 0.077686 & $\mathrm{H}$ & 3.923472 & 1.453503 & -1.39038 \\
\hline $\mathrm{H}$ & -6.13107 & -1.20525 & -0.88658 & $\mathrm{H}$ & 1.603414 & 1.708488 & -0.9239 \\
\hline $\mathrm{H}$ & 2.807901 & 1.013832 & 1.615881 & $\mathrm{H}$ & -5.0288 & 1.443761 & 0.595079 \\
\hline $\mathrm{H}$ & 4.284834 & 2.959136 & 1.95272 & $\mathrm{H}$ & -7.42555 & 1.071108 & 0.254849 \\
\hline $\mathrm{H}$ & 3.825426 & 5.100456 & 0.758395 & $\mathrm{H}$ & -8.23154 & -1.04773 & -0.79057 \\
\hline \multirow[t]{15}{*}{$\mathrm{H}$} & 1.82034 & 5.290106 & -0.69892 & $\mathrm{H}$ & -6.58507 & -2.80533 & -1.41192 \\
\hline & & & & $\mathrm{H}$ & 0.996094 & 1.838824 & 1.967785 \\
\hline & & & & $\mathrm{H}$ & 1.810646 & 4.122108 & 2.435128 \\
\hline & & & & $\mathrm{H}$ & 0.957482 & 6.05147 & 1.103159 \\
\hline & & & & $\mathrm{H}$ & -0.78247 & 5.673849 & -0.63281 \\
\hline & & & & 1 & 5.782292 & 1.084127 & -1.83617 \\
\hline & & & & $\mathrm{H}$ & 7.191224 & 0.088341 & -1.64822 \\
\hline & & & & $\mathrm{H}$ & 6.151488 & -2.02311 & 0.692484 \\
\hline & & & & $\mathrm{H}$ & 5.475751 & -2.80696 & -0.7342 \\
\hline & & & & $\mathrm{H}$ & 7.828928 & -3.22185 & -0.6821 \\
\hline & & & & $\mathrm{H}$ & 7.512323 & -2.17441 & -2.07354 \\
\hline & & & & $\mathrm{H}$ & 8.25478 & -1.51228 & -0.59698 \\
\hline & & & & $\mathrm{H}$ & 7.34052 & 2.084812 & -0.16357 \\
\hline & & & & $\mathrm{H}$ & 7.27485 & 0.612215 & 0.823683 \\
\hline & & & & $\mathrm{H}$ & 5.83012 & 1.625653 & 0.649809 \\
\hline
\end{tabular}

SI Table S10: Number of imaginary frequencies obtained for optimized ground state geometries of P1, P2, PBC1, and PBC2 at B3LYP/6-31+G* level of theory

\begin{tabular}{|c|c|c|c|}
\hline \multirow{2}{*}{ Systems } & \multicolumn{3}{|c|}{ Imaginary Frequencies } \\
\cline { 2 - 4 } & Gas Phase & Aniline & TEA \\
\hline P1 & 0 & 0 & 0 \\
\hline P2 & 0 & 0 & 0 \\
\hline PBC1 & - & 0 & 0 \\
\hline PBC2 & - & 0 & 0 \\
\hline
\end{tabular}


SI Table S11: The absolute energy of the optimized ground state $\left(\mathrm{S}_{0}\right)$ and singlet excited state $\left(\mathrm{S}_{1}\right)$ geometries of $\mathrm{P} 1, \mathrm{P} 2, \mathrm{PBC} 1$, and $\mathrm{PBC} 2$ at $\mathrm{B} 3 \mathrm{LYP} / 6-31+\mathrm{G}^{*}$ level of theory. The relative energy is expressed in $\mathrm{eV}$ with respective to their ground state.

\begin{tabular}{|c|c|c|c|}
\hline \multirow{2}{*}{ Systems } & \multicolumn{2}{|c|}{ Absolute Energy (a.u) } & \multirow{2}{*}{ Relative Energy (eV) } \\
\cline { 2 - 3 } & $\mathrm{S}_{0}$ & $\mathrm{~S}_{1}$ & 0.43 \\
\hline P1 in aniline & -1171.5836567 & -1171.568023 & 0.40 \\
\hline P1 in TEA & -1171.5801174 & -1171.565409 & 0.22 \\
\hline P2 in aniline & -1384.185914 & -1384.177688 & 0.20 \\
\hline P2 in TEA & -1384.181681 & -1384.174262 & 0.56 \\
\hline PBC1 in aniline & -1395.730024 & -1395.709422 & 0.35 \\
\hline PBC1 in TEA & -1395.723909 & -1395.711195 & 0.01 \\
\hline PBC2 in aniline & -1608.324801 & -1608.324473 & 0.1 \\
\hline PBC2 in TEA & -1608.318092 & -1608.31454 & \\
\hline
\end{tabular}

SI Table S12: Cartesian coordinates (in Å units) of the optimized singlet state geometries of P1 and $\mathrm{P} 2$ in aniline at B3LYP/6-31+G* level of theory

\begin{tabular}{|c|c|c|c|c|c|c|c|}
\hline \multicolumn{4}{|c|}{$\mathrm{P} 1$ (in aniline) } & \multicolumn{4}{|c|}{ P2 (in aniline) } \\
\hline C & -1.78431 & -0.14136 & 0.011392 & $\mathrm{C}$ & -2.98872 & -0.43551 & -0.01761 \\
\hline C & -1.32957 & -1.46058 & 0.233247 & $\mathrm{C}$ & -2.22583 & -1.56047 & 0.364714 \\
\hline$N$ & -0.03942 & -1.78275 & 0.167299 & $\mathrm{~N}$ & -0.89624 & -1.52831 & 0.419611 \\
\hline C & 0.907935 & -0.83454 & -0.01563 & $\mathrm{C}$ & -0.21158 & -0.37752 & 0.207797 \\
\hline C & 0.566921 & 0.536198 & 0.109769 & $\mathrm{C}$ & -0.91835 & 0.857486 & 0.18918 \\
\hline C & -0.80941 & 0.87791 & 0.05234 & $\mathrm{C}$ & -2.32173 & 0.809829 & -0.00422 \\
\hline C & -3.24893 & 0.038122 & -0.19004 & $\mathrm{C}$ & -4.42332 & -0.67375 & -0.33715 \\
\hline C & -4.12777 & -0.84997 & 0.475878 & $\mathrm{C}$ & -5.09672 & -1.7124 & 0.351102 \\
\hline C & -3.52522 & -1.96215 & 1.304377 & $\mathrm{C}$ & -4.30248 & -2.55289 & 1.325236 \\
\hline C & -2.32227 & -2.5526 & 0.552905 & $\mathrm{C}$ & -2.92212 & -2.85271 & 0.721025 \\
\hline C & 1.553177 & 1.622816 & 0.367334 & $\mathrm{C}$ & -0.28893 & 2.183486 & 0.446414 \\
\hline C & 1.244578 & 2.939014 & -0.04457 & $\mathrm{C}$ & -0.89222 & 3.344186 & -0.08868 \\
\hline C & -0.11856 & 3.18842 & -0.64272 & $\mathrm{C}$ & -2.19744 & 3.179063 & -0.82767 \\
\hline C & -1.14436 & 2.354618 & 0.137153 & $\mathrm{C}$ & -3.04438 & 2.141814 & -0.07815 \\
\hline C & 2.250557 & -1.36313 & -0.37284 & $\mathrm{C}$ & 1.238289 & -0.54607 & -0.00924 \\
\hline C & 2.690961 & -2.6341 & 0.083941 & C & 1.95832 & -1.65507 & 0.50939 \\
\hline C & 3.969278 & -3.10324 & -0.25218 & C & 3.335322 & -1.79195 & 0.310205 \\
\hline C & 4.797083 & -2.35945 & -1.08874 & $\mathrm{C}$ & 4.071153 & -0.8777 & -0.47424 \\
\hline C & 4.352162 & -1.1368 & -1.61027 & C & 3.339633 & 0.202391 & -1.04616 \\
\hline C & 3.09674 & -0.65635 & -1.25003 & C & 1.982856 & 0.342087 & -0.81385 \\
\hline C & -3.79356 & 0.973102 & -1.08909 & C & -5.11019 & 0.006123 & -1.3597 \\
\hline C & -5.17432 & 1.087409 & -1.26285 & C & -6.45031 & -0.27095 & -1.6383 \\
\hline C & -6.03932 & 0.252853 & -0.55022 & C & -7.12796 & -1.25093 & -0.90832 \\
\hline C & -5.50906 & -0.72171 & 0.299968 & C & -6.44042 & -1.97675 & 0.06876 \\
\hline
\end{tabular}




\begin{tabular}{|c|c|c|c|c|c|c|c|}
\hline C & 2.743751 & 1.404375 & 1.085149 & $\mathrm{C}$ & 0.831897 & 2.336135 & 1.28416 \\
\hline C & 3.639625 & 2.445539 & 1.326475 & C & 1.382112 & 3.593994 & 1.529028 \\
\hline C & 3.358395 & 3.733736 & 0.859354 & C & 0.817123 & 4.73265 & 0.944639 \\
\hline C & 2.156407 & 3.974066 & 0.189414 & $\mathrm{C}$ & -0.3246 & 4.600509 & 0.150722 \\
\hline 0 & 1.91529 & -3.43708 & 0.862724 & 0 & 1.354172 & -2.62675 & 1.248702 \\
\hline $\mathrm{H}$ & -3.18766 & -1.57851 & 2.279085 & $\mathrm{~N}$ & 5.429916 & -1.05113 & -0.71811 \\
\hline $\mathrm{H}$ & -4.27266 & -2.73645 & 1.506079 & C & 6.23926 & 0.098644 & -1.14127 \\
\hline $\mathrm{H}$ & -1.82194 & -3.32759 & 1.142843 & $\mathrm{C}$ & 6.117266 & -2.15997 & -0.03642 \\
\hline $\mathrm{H}$ & -2.66695 & -3.02851 & -0.37777 & $\mathrm{C}$ & 7.532758 & -2.45189 & -0.53589 \\
\hline $\mathrm{H}$ & -0.37022 & 4.25297 & -0.58904 & $\mathrm{C}$ & 6.570485 & 1.089518 & -0.01623 \\
\hline $\mathrm{H}$ & -0.14454 & 2.903182 & -1.7057 & $\mathrm{H}$ & -4.16859 & -2.01743 & 2.277445 \\
\hline $\mathrm{H}$ & -1.10124 & 2.650338 & 1.197663 & $\mathrm{H}$ & -4.83596 & -3.48199 & 1.551888 \\
\hline $\mathrm{H}$ & -2.15737 & 2.565335 & -0.19701 & $\mathrm{H}$ & -2.29301 & -3.41613 & 1.418078 \\
\hline $\mathrm{H}$ & 4.281282 & -4.06898 & 0.135618 & $\mathrm{H}$ & -3.04154 & -3.47424 & -0.17954 \\
\hline $\mathrm{H}$ & 5.779729 & -2.74279 & -1.35177 & $\mathrm{H}$ & -2.72707 & 4.13594 & -0.88811 \\
\hline $\mathrm{H}$ & 4.977894 & -0.56621 & -2.29065 & $\mathrm{H}$ & -2.02853 & 2.836856 & -1.86042 \\
\hline $\mathrm{H}$ & 2.75452 & 0.290846 & -1.6547 & $\mathrm{H}$ & -3.20452 & 2.498757 & 0.952073 \\
\hline $\mathrm{H}$ & -3.13726 & 1.594432 & -1.68996 & $\mathrm{H}$ & -4.03171 & 2.046765 & -0.52372 \\
\hline $\mathrm{H}$ & -5.56964 & 1.817504 & -1.9643 & $\mathrm{H}$ & 3.800939 & -2.64814 & 0.780021 \\
\hline $\mathrm{H}$ & -7.11534 & 0.338686 & -0.6773 & $\mathrm{H}$ & 3.824517 & 0.928401 & -1.6861 \\
\hline $\mathrm{H}$ & -6.17526 & -1.40507 & 0.822311 & $\mathrm{H}$ & 1.469847 & 1.175205 & -1.28349 \\
\hline $\mathrm{H}$ & 2.971147 & 0.412981 & 1.462549 & $\mathrm{H}$ & -4.58848 & 0.733657 & -1.97341 \\
\hline $\mathrm{H}$ & 4.552459 & 2.251423 & 1.883694 & $\mathrm{H}$ & -6.95667 & 0.269326 & -2.4341 \\
\hline $\mathrm{H}$ & 4.055266 & 4.548363 & 1.03905 & $\mathrm{H}$ & -8.17201 & -1.4695 & -1.11712 \\
\hline $\mathrm{H}$ & 1.909397 & 4.981912 & -0.13794 & $\mathrm{H}$ & -6.94826 & -2.77268 & 0.609532 \\
\hline \multirow[t]{15}{*}{$\mathrm{H}$} & 0.986547 & -3.09111 & 0.777208 & $\mathrm{H}$ & 1.278178 & 1.463347 & 1.749191 \\
\hline & & & & $\mathrm{H}$ & 2.248307 & 3.684359 & 2.179635 \\
\hline & & & & $\mathrm{H}$ & 1.245285 & 5.715078 & 1.126814 \\
\hline & & & & $\mathrm{H}$ & -0.79746 & 5.484629 & -0.27218 \\
\hline & & & & $\mathrm{H}$ & 0.374478 & -2.5107 & 1.103907 \\
\hline & & & & $\mathrm{H}$ & 7.161506 & -0.28567 & -1.58026 \\
\hline & & & & $\mathrm{H}$ & 5.725553 & 0.612099 & -1.95718 \\
\hline & & & & $\mathrm{H}$ & 6.141996 & -1.9897 & 1.053368 \\
\hline & & & & $\mathrm{H}$ & 5.520517 & -3.06218 & -0.20014 \\
\hline & & & & $\mathrm{H}$ & 7.880175 & -3.37423 & -0.05696 \\
\hline & & & & $\mathrm{H}$ & 7.553097 & -2.60892 & -1.62048 \\
\hline & & & & $\mathrm{H}$ & 8.249317 & -1.66402 & -0.28234 \\
\hline & & & & $\mathrm{H}$ & 7.17477 & 1.916367 & -0.4094 \\
\hline & & & & $\mathrm{H}$ & 5.659573 & 1.511552 & 0.422559 \\
\hline & & & & $\mathrm{H}$ & 7.141583 & 0.605002 & 0.784616 \\
\hline
\end{tabular}


SI Table S13: Cartesian coordinates (in $\AA$ units) of the optimized singlet state geometries of P1 and $\mathrm{P} 2$ in TEA at B3LYP/6-31+G* level of theory.

\begin{tabular}{|c|c|c|c|c|c|c|c|}
\hline \multicolumn{4}{|c|}{ P1 (in TEA) } & \multicolumn{4}{|c|}{$\mathrm{P} 2$ (in TEA) } \\
\hline$C$ & 1.731177 & -0.10828 & -0.01955 & $C$ & 2.93877 & -0.43866 & 0.001422 \\
\hline C & 1.281229 & -1.35708 & -0.60436 & $C$ & 2.189262 & -1.49692 & -0.6465 \\
\hline$N$ & 0.01136 & -1.69401 & -0.66597 & $\mathrm{~N}$ & 0.890061 & -1.42491 & -0.82721 \\
\hline C & -0.94452 & -0.83756 & -0.15394 & $C$ & 0.189933 & -0.30186 & -0.44322 \\
\hline C & -0.59904 & 0.594965 & -0.09368 & $C$ & 0.933724 & 0.937665 & -0.23484 \\
\hline C & 0.758539 & 0.91879 & 0.086076 & $\mathrm{C}$ & 2.303732 & 0.825652 & 0.07745 \\
\hline C & 3.160929 & 0.022397 & 0.271134 & C & 4.306375 & -0.75096 & 0.428721 \\
\hline C & 4.096723 & -0.70901 & -0.51521 & $C$ & 5.044538 & -1.73555 & -0.29058 \\
\hline C & 3.563094 & -1.58292 & -1.62378 & $\mathrm{C}$ & 4.372308 & -2.41047 & -1.46193 \\
\hline C & 2.304134 & -2.32345 & -1.14274 & $C$ & 2.915057 & -2.74012 & -1.09772 \\
\hline C & -1.562 & 1.639986 & -0.39216 & $C$ & 0.36233 & 2.253611 & -0.49975 \\
\hline C & -1.28361 & 2.984124 & 0.001951 & $C$ & 0.963844 & 3.410504 & 0.075173 \\
\hline C & 0.007849 & 3.238375 & 0.733499 & $C$ & 2.201013 & 3.20207 & 0.909942 \\
\hline C & 1.127248 & 2.393019 & 0.117738 & $C$ & 3.07456 & 2.123072 & 0.260945 \\
\hline C & -2.15143 & -1.40424 & 0.349395 & C & -1.21606 & -0.486 & -0.16439 \\
\hline C & -2.52236 & -2.78687 & 0.070904 & $C$ & -1.94453 & -1.65373 & -0.61866 \\
\hline C & -3.72672 & -3.31073 & 0.542449 & $C$ & -3.29318 & -1.82164 & -0.33978 \\
\hline C & -4.58668 & -2.53335 & 1.32423 & $C$ & -4.02512 & -0.8851 & 0.43706 \\
\hline C & -4.23929 & -1.20864 & 1.649428 & $\mathrm{C}$ & -3.31054 & 0.251893 & 0.919479 \\
\hline C & -3.06004 & -0.65721 & 1.176839 & $\mathrm{C}$ & -1.97151 & 0.430809 & 0.629466 \\
\hline C & 3.655972 & 0.782403 & 1.358205 & $C$ & 4.905935 & -0.18838 & 1.582087 \\
\hline C & 5.020077 & 0.862689 & 1.621692 & C & 6.199298 & -0.53027 & 1.967426 \\
\hline$C$ & 5.934985 & 0.173656 & 0.814747 & $C$ & 6.933833 & -1.46265 & 1.223314 \\
\hline$C$ & 5.461257 & -0.61759 & -0.23749 & $\mathrm{C}$ & 6.339061 & -2.06811 & 0.109832 \\
\hline C & -2.7734 & 1.377546 & -1.09237 & $C$ & -0.77379 & 2.433075 & -1.33459 \\
\hline C & -3.66583 & 2.405517 & -1.38329 & $C$ & -1.30474 & 3.702706 & -1.55785 \\
\hline$C$ & -3.38865 & 3.714376 & -0.97583 & $C$ & -0.72546 & 4.826019 & -0.9604 \\
\hline$C$ & -2.19553 & 3.992602 & -0.28658 & $\mathrm{C}$ & 0.414944 & 4.6685 & -0.1528 \\
\hline 0 & -1.73901 & -3.58512 & -0.67611 & 0 & -1.34271 & -2.60391 & -1.36454 \\
\hline $\mathrm{H}$ & 3.302124 & -0.96944 & -2.49964 & $\mathrm{~N}$ & -5.36321 & -1.08336 & 0.72846 \\
\hline $\mathrm{H}$ & 4.325945 & -2.29742 & -1.95114 & $C$ & -6.16644 & 0.001835 & 1.299178 \\
\hline $\mathrm{H}$ & 1.850959 & -2.91705 & -1.94341 & $\mathrm{C}$ & -6.04915 & -2.25114 & 0.147447 \\
\hline $\mathrm{H}$ & 2.585839 & -3.02521 & -0.34219 & $C$ & -7.44646 & -2.54057 & 0.694084 \\
\hline $\mathrm{H}$ & 0.265846 & 4.302663 & 0.702246 & $\mathrm{C}$ & -6.62416 & 1.044737 & 0.267654 \\
\hline $\mathrm{H}$ & -0.10562 & 2.96592 & 1.795221 & $\mathrm{H}$ & 4.378105 & -1.74662 & -2.34017 \\
\hline $\mathrm{H}$ & 1.294993 & 2.718429 & -0.92211 & $\mathrm{H}$ & 4.915027 & -3.31937 & -1.74463 \\
\hline $\mathrm{H}$ & 2.065588 & 2.564921 & 0.64272 & $\mathrm{H}$ & 2.379075 & -3.18547 & -1.94274 \\
\hline $\mathrm{H}$ & -3.96549 & -4.34088 & 0.294029 & $\mathrm{H}$ & 2.909792 & -3.48322 & -0.2848 \\
\hline $\mathrm{H}$ & -5.51623 & -2.96079 & 1.691097 & $\mathrm{H}$ & 2.758659 & 4.139812 & 1.01391 \\
\hline
\end{tabular}




\begin{tabular}{|rrrr|rrrr|}
\hline $\mathrm{H}$ & -4.89466 & -0.61435 & 2.280831 & $\mathrm{H}$ & 1.923326 & 2.877223 & 1.925986 \\
$\mathrm{H}$ & -2.78769 & 0.349393 & 1.470622 & $\mathrm{H}$ & 3.385816 & 2.470493 & -0.73809 \\
$\mathrm{H}$ & 2.957503 & 1.272859 & 2.029448 & $\mathrm{H}$ & 3.991001 & 1.976292 & 0.829534 \\
$\mathrm{H}$ & 5.37096 & 1.445975 & 2.469433 & $\mathrm{H}$ & -3.75711 & -2.7066 & -0.75428 \\
$\mathrm{H}$ & 7.001047 & 0.232447 & 1.017468 & $\mathrm{H}$ & -3.8004 & 1.000317 & 1.528557 \\
$\mathrm{H}$ & 6.164234 & -1.17748 & -0.85124 & $\mathrm{H}$ & -1.46575 & 1.291865 & 1.049093 \\
$\mathrm{H}$ & -2.98175 & 0.371222 & -1.43753 & $\mathrm{H}$ & 4.330932 & 0.48618 & 2.209136 \\
$\mathrm{H}$ & -4.57839 & 2.186025 & -1.93131 & $\mathrm{H}$ & 6.628438 & -0.08538 & 2.862151 \\
$\mathrm{H}$ & -4.08692 & 4.517326 & -1.19687 & $\mathrm{H}$ & 7.943535 & -1.73385 & 1.520419 \\
$\mathrm{H}$ & -1.97708 & 5.012305 & 0.023653 & $\mathrm{H}$ & 6.890309 & -2.81724 & -0.45574 \\
$\mathrm{H}$ & -0.8834 & -3.0789 & -0.85465 & $\mathrm{H}$ & -1.20691 & 1.575053 & -1.83745 \\
& & & & $\mathrm{H}$ & -2.1681 & 3.815828 & -2.20949 \\
& & & & $\mathrm{H}$ & -1.13872 & 5.816595 & -1.13253 \\
& & & $\mathrm{H}$ & 0.884578 & 5.541935 & 0.295821 \\
& & & $\mathrm{H}$ & -0.3521 & -2.40612 & -1.33407 \\
& & & $\mathrm{H}$ & -7.03409 & -0.44281 & 1.787677 \\
& & & $\mathrm{H}$ & -5.59487 & 0.481478 & 2.097471 \\
& & & $\mathrm{H}$ & -6.10266 & -2.13986 & -0.94794 \\
& & & $\mathrm{H}$ & -5.42595 & -3.12886 & 0.344616 \\
& & & $\mathrm{H}$ & -7.79508 & -3.47622 & 0.243051 \\
& & & $\mathrm{H}$ & -7.43997 & -2.67857 & 1.780968 \\
& & & $\mathrm{H}$ & -8.1765 & -1.76527 & 0.440398 \\
& & & $\mathrm{H}$ & -7.21646 & 1.823652 & 0.763111 \\
& & & -5.76741 & 1.52151 & -0.2198 \\
& & & -7.24723 & 0.586248 & -0.50877 \\
\hline
\end{tabular}

SI Table S14: Cartesian coordinates (in $\AA$ units) of the optimized singlet state geometries of $\mathrm{PBC} 1$ and $\mathrm{PBC} 2$ in aniline at B3LYP/6-31+G* level of theory.

\begin{tabular}{|c|c|c|c|c|c|c|c|}
\hline \multicolumn{4}{|c|}{ PBC1 (in aniline) } & \multicolumn{4}{|c|}{ PBC2 (in aniline) } \\
\hline C & -1.78229 & 0.149834 & -0.08339 & $\mathrm{C}$ & -2.96782 & -0.14811 & -0.0585 \\
\hline C & -1.31607 & -1.18192 & 0.116935 & $\mathrm{C}$ & -2.21767 & -1.33176 & -0.0229 \\
\hline $\mathrm{N}$ & -0.01337 & -1.46711 & 0.318751 & $\mathrm{~N}$ & -0.85626 & -1.32353 & 0.188543 \\
\hline C & 0.957741 & -0.47568 & 0.019115 & $\mathrm{C}$ & -0.17795 & -0.08217 & 0.158793 \\
\hline C & 0.538827 & 0.905758 & 0.037271 & $\mathrm{C}$ & -0.87755 & 1.11292 & 0.160684 \\
\hline C & -0.81976 & 1.217221 & -0.11604 & C & -2.30549 & 1.120629 & -0.0119 \\
\hline C & -3.24617 & 0.341589 & -0.12067 & C & -4.44356 & -0.2964 & -0.08491 \\
\hline C & -4.06747 & -0.71097 & -0.61367 & $\mathrm{C}$ & -5.01894 & -1.44854 & -0.67737 \\
\hline C & -3.39367 & -2.00303 & -0.98022 & $\mathrm{C}$ & -4.07919 & -2.50025 & -1.20773 \\
\hline C & -2.30858 & -2.31923 & 0.057482 & $\mathrm{C}$ & -2.90221 & -2.66241 & -0.23835 \\
\hline C & 1.489672 & 1.986404 & 0.308993 & C & -0.20907 & 2.42247 & 0.355493 \\
\hline C & 1.212396 & 3.289413 & -0.18179 & $\mathrm{C}$ & -0.75263 & 3.560062 & -0.28348 \\
\hline
\end{tabular}




\begin{tabular}{|c|c|c|c|c|c|c|c|}
\hline C & -0.04848 & 3.458855 & -0.98395 & $\mathrm{C}$ & -2.00313 & 3.366529 & -1.10172 \\
\hline C & -1.18949 & 2.672234 & -0.32554 & $\mathrm{C}$ & -2.97088 & 2.437764 & -0.34225 \\
\hline C & 2.196853 & -0.95431 & -0.50348 & $\mathrm{C}$ & 1.272398 & -0.23042 & -0.06819 \\
\hline C & 2.564041 & -2.3492 & -0.31146 & $\mathrm{C}$ & 1.941201 & -1.33448 & 0.539284 \\
\hline C & 3.756081 & -2.85864 & -0.84411 & $\mathrm{C}$ & 3.312482 & -1.50398 & 0.421895 \\
\hline C & 4.605954 & -2.03861 & -1.57947 & $\mathrm{C}$ & 4.090142 & -0.61332 & -0.36739 \\
\hline C & 4.262949 & -0.68594 & -1.80447 & C & 3.39819 & 0.43725 & -1.06078 \\
\hline C & 3.090051 & -0.16076 & -1.29103 & $\mathrm{C}$ & 2.034273 & 0.596017 & -0.92241 \\
\hline C & -3.89601 & 1.49896 & 0.371382 & $\mathrm{C}$ & -5.30665 & 0.637016 & 0.525906 \\
\hline C & -5.28046 & 1.639675 & 0.302191 & $\mathrm{C}$ & -6.6918 & 0.466803 & 0.500986 \\
\hline C & -6.06753 & 0.624345 & -0.25192 & $\mathrm{C}$ & -7.2514 & -0.64625 & -0.13333 \\
\hline C & -5.45101 & -0.5525 & -0.68928 & $\mathrm{C}$ & -6.4063 & -1.60306 & -0.70713 \\
\hline C & 2.642547 & 1.782487 & 1.10599 & $\mathrm{C}$ & 0.881519 & 2.588248 & 1.230263 \\
\hline C & 3.503816 & 2.838791 & 1.388005 & C & 1.467795 & 3.840146 & 1.414516 \\
\hline C & 3.238928 & 4.114063 & 0.874534 & $\mathrm{C}$ & 0.957075 & 4.955206 & 0.740349 \\
\hline C & 2.093088 & 4.332063 & 0.095089 & $\mathrm{C}$ & -0.15657 & 4.81086 & -0.09312 \\
\hline $\mathrm{O}$ & 1.788513 & -3.16933 & 0.388529 & 0 & 1.214384 & -2.20244 & 1.256187 \\
\hline B & 0.600736 & -2.69332 & 1.112431 & B & -0.08788 & -2.59706 & 0.692271 \\
\hline$F$ & 0.981735 & -2.19841 & 2.376653 & $\mathrm{~F}$ & -0.8119 & -3.23332 & 1.71102 \\
\hline$F$ & -0.26738 & -3.7666 & 1.261881 & $\mathrm{~F}$ & 0.123248 & -3.50489 & -0.37862 \\
\hline $\mathrm{H}$ & -4.11953 & -2.82168 & -1.01819 & $\mathrm{~N}$ & 5.444885 & -0.77199 & -0.4875 \\
\hline $\mathrm{H}$ & -2.93085 & -1.93588 & -1.97649 & $\mathrm{C}$ & 6.265237 & 0.208571 & -1.21507 \\
\hline $\mathrm{H}$ & -2.77166 & -2.46504 & 1.044372 & C & 6.10847 & -1.85554 & 0.276019 \\
\hline $\mathrm{H}$ & -1.79717 & -3.2456 & -0.19435 & C & 7.593115 & -2.06668 & -0.00448 \\
\hline $\mathrm{H}$ & 0.105243 & 3.083672 & -2.00735 & C & 6.620032 & 1.44191 & -0.37217 \\
\hline $\mathrm{H}$ & -0.31838 & 4.516905 & -1.06601 & $\mathrm{H}$ & -4.60149 & -3.45514 & -1.33415 \\
\hline $\mathrm{H}$ & -2.08261 & 2.737139 & -0.95281 & $\mathrm{H}$ & -3.69353 & -2.20834 & -2.19693 \\
\hline $\mathrm{H}$ & -1.44032 & 3.154804 & 0.631711 & $\mathrm{H}$ & -3.2661 & -3.06101 & 0.720545 \\
\hline $\mathrm{H}$ & 3.99086 & -3.90303 & -0.66124 & $\mathrm{H}$ & -2.18785 & -3.38855 & -0.62934 \\
\hline $\mathrm{H}$ & 5.527215 & -2.44223 & -1.99045 & $\mathrm{H}$ & -1.76101 & 2.911901 & -2.07402 \\
\hline $\mathrm{H}$ & 4.915137 & -0.05576 & -2.40282 & $\mathrm{H}$ & -2.48034 & 4.331922 & -1.30648 \\
\hline $\mathrm{H}$ & 2.823834 & 0.863509 & -1.51984 & $\mathrm{H}$ & -3.8604 & 2.264381 & -0.95621 \\
\hline $\mathrm{H}$ & -3.32614 & 2.282129 & 0.852761 & $\mathrm{H}$ & -3.31752 & 2.975612 & 0.556878 \\
\hline $\mathrm{H}$ & -5.74561 & 2.539905 & 0.695393 & $\mathrm{H}$ & 3.750364 & -2.34499 & 0.940864 \\
\hline $\mathrm{H}$ & -7.14692 & 0.733528 & -0.31373 & $\mathrm{H}$ & 3.93118 & 1.12207 & -1.70563 \\
\hline $\mathrm{H}$ & -6.05499 & -1.36871 & -1.07966 & $\mathrm{H}$ & 1.542237 & 1.395079 & -1.46566 \\
\hline $\mathrm{H}$ & 2.836887 & 0.799553 & 1.5221 & $\mathrm{H}$ & -4.89347 & 1.490708 & 1.050853 \\
\hline $\mathrm{H}$ & 4.377988 & 2.671183 & 2.010956 & $\mathrm{H}$ & -7.33145 & 1.200707 & 0.985097 \\
\hline $\mathrm{H}$ & 3.911836 & 4.939828 & 1.089509 & $\mathrm{H}$ & -8.32958 & -0.78161 & -0.16118 \\
\hline \multirow[t]{4}{*}{$\mathrm{H}$} & 1.88202 & 5.326492 & -0.29136 & $\mathrm{H}$ & -6.82972 & -2.49154 & -1.17149 \\
\hline & & & & $\mathrm{H}$ & 1.257804 & 1.73332 & 1.784278 \\
\hline & & & & $\mathrm{H}$ & 2.308268 & 3.948936 & 2.095157 \\
\hline & & & & $\mathrm{H}$ & 1.405085 & 5.935155 & 0.884456 \\
\hline
\end{tabular}




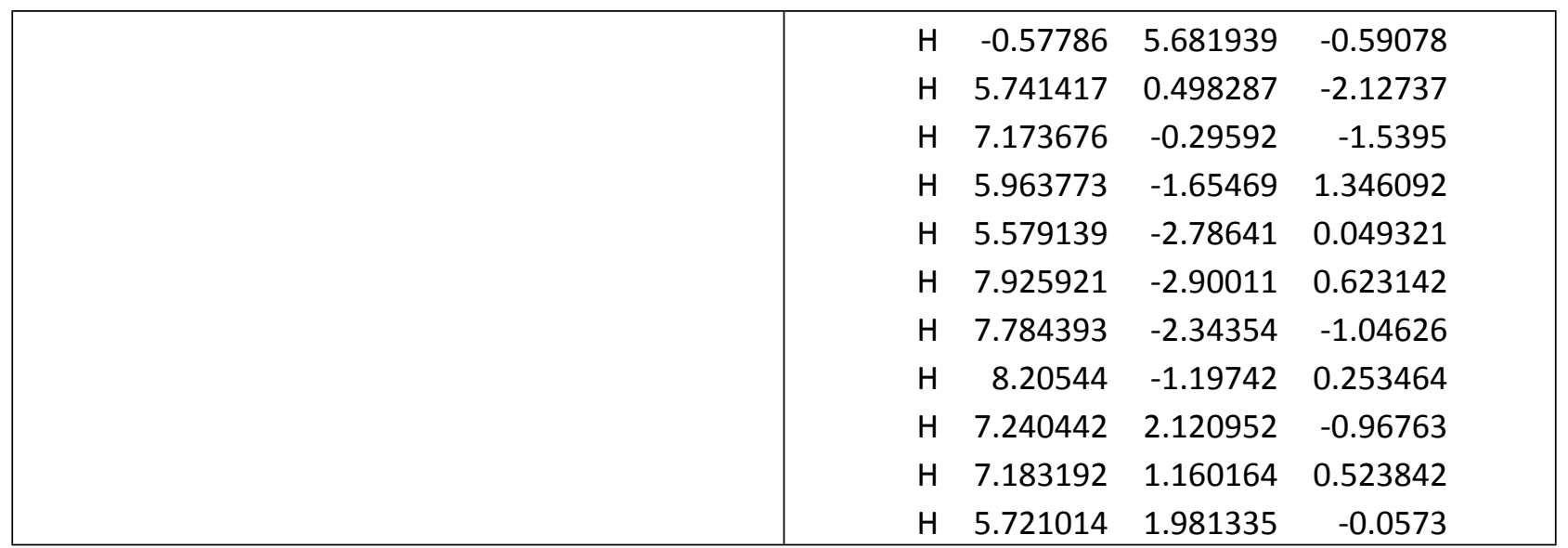

SI Table S15: Cartesian coordinates (in $\AA$ units) of the optimized singlet state geometries of PBC1 and PBC2 in TEA at B3LYP/6-31+G* level of theory.

\begin{tabular}{|c|c|c|c|c|c|c|c|}
\hline \multicolumn{4}{|c|}{ PBC1 (inTEA) } & \multicolumn{4}{|c|}{ PBC2 (in TEA) } \\
\hline C & -1.80015 & 0.166345 & -0.04004 & $\mathrm{C}$ & -2.96989 & -0.14398 & -0.04051 \\
\hline C & -1.34738 & -1.1588 & -0.00276 & C & -2.22135 & -1.32994 & -0.03272 \\
\hline $\mathrm{N}$ & -0.02128 & -1.47449 & 0.11029 & $\mathrm{~N}$ & -0.85205 & -1.31846 & 0.171297 \\
\hline C & 0.945432 & -0.43867 & 0.050737 & C & -0.18846 & -0.08172 & 0.154109 \\
\hline C & 0.535646 & 0.900487 & 0.060095 & C & -0.87294 & 1.111076 & 0.17863 \\
\hline C & -0.83358 & 1.237032 & -0.06965 & C & -2.31859 & 1.119752 & 0.027502 \\
\hline C & -3.26253 & 0.383173 & 0.02252 & C & -4.44817 & -0.29664 & -0.07506 \\
\hline C & -4.13571 & -0.61157 & -0.4898 & C & -5.01717 & -1.43524 & -0.69525 \\
\hline C & -3.51111 & -1.87819 & -1.01327 & C & -4.07507 & -2.47493 & -1.24677 \\
\hline C & -2.35206 & -2.28637 & -0.09682 & C & -2.89801 & -2.65741 & -0.28012 \\
\hline C & 1.494486 & 2.017804 & 0.252815 & $\mathrm{C}$ & -0.19921 & 2.418978 & 0.357884 \\
\hline C & 1.223242 & 3.257656 & -0.36697 & C & -0.75235 & 3.555025 & -0.27621 \\
\hline C & -0.05867 & 3.370628 & -1.15101 & C & -2.01688 & 3.360874 & -1.07274 \\
\hline C & -1.18837 & 2.677371 & -0.36968 & $\mathrm{C}$ & -2.97941 & 2.44058 & -0.29239 \\
\hline C & 2.302766 & -0.90219 & -0.29222 & $\mathrm{C}$ & 1.272143 & -0.23741 & -0.07225 \\
\hline C & 2.740089 & -2.17364 & 0.236328 & C & 1.937992 & -1.32837 & 0.563435 \\
\hline C & 4.071716 & -2.63661 & 0.053775 & C & 3.313064 & -1.49202 & 0.454363 \\
\hline C & 4.943769 & -1.90509 & -0.73053 & C & 4.083285 & -0.62454 & -0.36179 \\
\hline C & 4.493614 & -0.71599 & -1.34444 & C & 3.388865 & 0.401307 & -1.09007 \\
\hline C & 3.186817 & -0.23745 & -1.13788 & C & 2.023075 & 0.561067 & -0.9537 \\
\hline C & -3.84099 & 1.517529 & 0.632009 & C & -5.31205 & 0.618562 & 0.557556 \\
\hline C & -5.22439 & 1.690659 & 0.675561 & C & -6.69691 & 0.446293 & 0.527561 \\
\hline C & -6.07241 & 0.730015 & 0.116839 & C & -7.25195 & -0.65211 & -0.13417 \\
\hline C & -5.51736 & -0.42295 & -0.44931 & C & -6.40456 & -1.59217 & -0.72985 \\
\hline C & 2.606895 & 1.910638 & 1.107686 & C & 0.904035 & 2.590572 & 1.215177 \\
\hline C & 3.473021 & 2.987547 & 1.290704 & C & 1.49485 & 3.84237 & 1.383133 \\
\hline C & 3.231801 & 4.197142 & 0.629515 & C & 0.973196 & 4.955661 & 0.714312 \\
\hline C & 2.102132 & 4.329211 & -0.18373 & $\mathrm{C}$ & -0.15431 & 4.80699 & -0.09849 \\
\hline
\end{tabular}




\begin{tabular}{|c|c|c|c|c|c|c|c|}
\hline $\mathrm{O}$ & 1.894303 & -2.91415 & 0.901858 & 0 & 1.211205 & -2.18674 & 1.275929 \\
\hline B & 0.422901 & -2.91329 & 0.535061 & B & -0.08956 & -2.5953 & 0.690744 \\
\hline $\mathrm{F}$ & -0.26529 & -3.33323 & 1.661872 & $\mathrm{~F}$ & -0.82618 & -3.22055 & 1.696219 \\
\hline $\mathrm{F}$ & 0.297943 & -3.82161 & -0.52231 & $\mathrm{~F}$ & 0.15139 & -3.49239 & -0.3748 \\
\hline $\mathrm{H}$ & -4.25261 & -2.68307 & -1.06229 & $\mathrm{~N}$ & 5.44111 & -0.7812 & -0.48027 \\
\hline $\mathrm{H}$ & -3.1282 & -1.72849 & -2.03483 & $C$ & 6.256161 & 0.174293 & -1.24481 \\
\hline $\mathrm{H}$ & -2.73747 & -2.51853 & 0.9065 & $C$ & 6.108949 & -1.83149 & 0.323391 \\
\hline $\mathrm{H}$ & -1.87495 & -3.19119 & -0.47235 & C & 7.597434 & -2.03971 & 0.059749 \\
\hline $\mathrm{H}$ & 0.045399 & 2.889596 & -2.13533 & $\mathrm{C}$ & 6.613918 & 1.437224 & -0.44843 \\
\hline $\mathrm{H}$ & -0.30817 & 4.422702 & -1.3304 & $\mathrm{H}$ & -4.59844 & -3.42665 & -1.39449 \\
\hline $\mathrm{H}$ & -2.11247 & 2.713981 & -0.95475 & $\mathrm{H}$ & -3.68922 & -2.16134 & -2.2293 \\
\hline $\mathrm{H}$ & -1.37853 & 3.261287 & 0.546472 & $\mathrm{H}$ & -3.2601 & -3.08657 & 0.666396 \\
\hline $\mathrm{H}$ & 4.349799 & -3.58066 & 0.510678 & $\mathrm{H}$ & -2.17852 & -3.36873 & -0.69007 \\
\hline $\mathrm{H}$ & 5.957995 & -2.2537 & -0.89847 & $\mathrm{H}$ & -1.7938 & 2.897998 & -2.04576 \\
\hline $\mathrm{H}$ & 5.168343 & -0.15077 & -1.98045 & $\mathrm{H}$ & -2.49255 & 4.327589 & -1.2773 \\
\hline $\mathrm{H}$ & 2.883531 & 0.687883 & -1.61392 & $\mathrm{H}$ & -3.88304 & 2.276869 & -0.88822 \\
\hline $\mathrm{H}$ & -3.20862 & 2.258539 & 1.106301 & $\mathrm{H}$ & -3.30106 & 2.985894 & 0.612122 \\
\hline $\mathrm{H}$ & -5.63868 & 2.573116 & 1.156945 & $\mathrm{H}$ & 3.750036 & -2.32294 & 0.989793 \\
\hline $\mathrm{H}$ & -7.15082 & 0.862707 & 0.143323 & $\mathrm{H}$ & 3.920339 & 1.07046 & -1.75239 \\
\hline $\mathrm{H}$ & -6.16823 & -1.19614 & -0.85291 & $\mathrm{H}$ & 1.527054 & 1.347035 & -1.51223 \\
\hline $\mathrm{H}$ & 2.781099 & 0.983458 & 1.645489 & $\mathrm{H}$ & -4.89845 & 1.459032 & 1.103583 \\
\hline $\mathrm{H}$ & 4.325435 & 2.888724 & 1.957752 & $\mathrm{H}$ & -7.33913 & 1.166049 & 1.029283 \\
\hline $\mathrm{H}$ & 3.902838 & 5.040816 & 0.769234 & $\mathrm{H}$ & -8.3298 & -0.7901 & -0.16587 \\
\hline \multirow[t]{15}{*}{$\mathrm{H}$} & 1.89004 & 5.280032 & -0.66819 & $\mathrm{H}$ & -6.82542 & -2.47091 & -1.21478 \\
\hline & & & & $\mathrm{H}$ & 1.285074 & 1.739272 & 1.771971 \\
\hline & & & & $\mathrm{H}$ & 2.34435 & 3.954081 & 2.052383 \\
\hline & & & & $\mathrm{H}$ & 1.421861 & 5.936742 & 0.849212 \\
\hline & & & & $\mathrm{H}$ & -0.58602 & 5.67635 & -0.59074 \\
\hline & & & & $\mathrm{H}$ & 5.727137 & 0.431271 & -2.164 \\
\hline & & & & $\mathrm{H}$ & 7.163608 & -0.34034 & -1.55708 \\
\hline & & & & $\mathrm{H}$ & 5.954855 & -1.59602 & 1.385134 \\
\hline & & & & $\mathrm{H}$ & 5.588271 & -2.77412 & 0.126493 \\
\hline & & & & $\mathrm{H}$ & 7.935676 & -2.84495 & 0.720346 \\
\hline & & & & $\mathrm{H}$ & 7.798246 & -2.35521 & -0.96928 \\
\hline & & & & $\mathrm{H}$ & 8.200778 & -1.15538 & 0.286745 \\
\hline & & & & $\mathrm{H}$ & 7.225804 & 2.099758 & -1.07088 \\
\hline & & & & $\mathrm{H}$ & 7.18662 & 1.188961 & 0.451519 \\
\hline & & & & $\mathrm{H}$ & 5.715463 & 1.982655 & -0.14259 \\
\hline
\end{tabular}




\section{References}

1. C. Lee, W. Yang, R.G. Parr, Development of the Colle-Salvetticorrelation-energy formula into a functional of the electron density, Phys.Rev. B. 1988, 37, 785-789.

2. A.D. Becke, Density-functional exchange-energy approximation with correctasymptotic behavior, Phys. Rev. A. 1988, 38, 3098-3100.

3. R. E. Stratmann, G. E. Scuseria, M. J. Frisch, J. Chem. Phys. 1998, 109, 8218-8224.

4. M. E. Casida, C. Jamorski, K. C. Casida, D. R. Salahub, J. Chem. Phys.1998, 108, 4439-4449.

5. M. J. Frisch, G. W. Trucks, H. B. Schlegel, G. E.Scuseria, M. A. Robb, J. R. Cheeseman, G. Scalmani, V. Barone, B. Mennucci, G. A.Petersson, H.Nakatsuji, M.Caricato, X. Li, H. P. Hratchian, A. F. Izmaylov, J.Bloino, G. Zheng, J. L.Sonnenberg, et al. Gaussian 09; Gaussian, Inc., Wallingford, CT, USA, 2009.

6. R. Dennington, T. Keith, J. Millam, GaussView, version 5.0.9, Semichem Inc., Shawnee Mission, KS, USA, 2009. 